THREE LECTURES ON MACROSCOPIC ASPECTS OF NUCLEAR DYNAMICS

\begin{tabular}{|c|c|}
\hline $\begin{array}{l}\text { Presented at the } \\
\text { International School of Nuclear Phys } \\
\text { "Ettore Majorana" Center for Sclentific } \\
\text { eld at Erice-Trapant, Sicily, } 26 \text { March to }\end{array}$ & $\begin{array}{l}\text { ure } \\
\text { ril } 1979\end{array}$ \\
\hline $\begin{array}{c}\text { by } \\
\text { W. J. Swiatecki } \\
\text { Nuclear Science Division } \\
\text { Lawrence Berkeley Laboratory } \\
\text { University of California } \\
\text { Berkeley, California } 94720\end{array}$ & 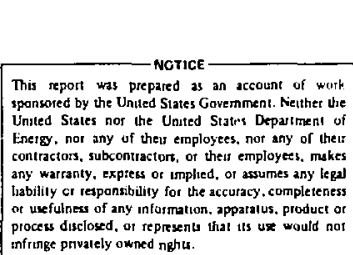 \\
\hline
\end{tabular}

LECTURE I .

Introduction

These lectures will concentrate on macroscopic aspects of nuclear dynamics. This means those aspects that come into prominence when the number of nucleons $A$ is large, $A \gg 1$.

The first lecture will deal with statics, i.e., it will discuss methods of treating the Potential Energs: Function of nuclear systems. From the Potential Energy the conservative forcis that drive the time evolution of a nuclear configuration can be deduced.

The second lecture will deal with dynamical aspects, especially with the nuclear Dissipation Function, which describes how disslpative frictional forces oppose t!ie conservative driving forces.

In the third lecture I will outline the kind of dynamics that results from the balance of these forces and I will describe a number of applications to nuclear fission and heavy-ion collisions of this "New Dynamics."

Thus the plan is as follows:

1. The Potential Energy

2. The Dissipation Function

3. The "New Dynamics" .

In preparing these lectures I soon realized that I will be concentrating so exclusively on one aspect of the nuclear problem that there is a risk of losing the right perspective on the true richness of nuclear physics. In order to counteract this danger to a certain extent, let me begin by placing the topic of these lectures in the broader framework of nuclear theory. I thought this could be done by listing the various approximations that can be made in treating the full nuclear many-body problem and pointing out the approximations especially relevant to the simplified treatment that I will describe. 
TABLE 1. Some approxtmations used in the nuclear many-body problem.

\begin{tabular}{ll}
\hline & $\begin{array}{c}\text { Small } \\
\text { parameter }\end{array}$ \\
Macroscopic & $\mathrm{A}^{-1} \ll 1$ \\
Leptodermous & $\mathrm{b} / \mathrm{R} \ll 1$ \\
$\begin{array}{l}\text { Dolichohodous (long mean-free path, } \\
\quad \text { independent particle) }\end{array}$ & $\mathrm{R} / \mathrm{L} \ll 1$ \\
Low temperature & $\mathrm{T} / \mathrm{E}_{\mathrm{F}} \ll 1$ \\
Sub-sonic & $\mathrm{V} / \mathrm{v}_{\mathrm{F}} \ll 1$ \\
Semi-classical & $\lambda_{\mathrm{F}} / \mathrm{R} \ll 1$ \\
\hline
\end{tabular}

The macroscopic aspect I already mentioned. More specifically, it will mean that the individual-particle degrees of freedom will not be considered explicitly.

"Leptodermous" means that the width b of the diffuse surface region is small compared to the size $R$ of the nuclear system. "Dolichohodous" is my attempt to make up a Greek word that would stand for "long-mean-free-path." The relative length of the nucleon mean free path, $L$, is the assumption that underlies the independent-particle or shell-model approximations to nuciear structure. I don't know Greek, so if dolichohodous does not have the right flavor I would like to have other suggestions. (I was attracted lo "dollchohodous" when I realized how unexpectedly close it was to the Polish "dalekochodzący," which conveys a similar idea and 1s, apparently, derived from common roots.) The assumption that the nuclear temperature $T$ is low compared to the Fermi energy $E_{F}$ means that the nucleons can be treated approximately as filling a nearly degenerate Fermi sea and obeying Fermi-Dirac statistics. "Sub-sonic" refers to the assumption that collective velocities $V$ will be assumed to be small compared to the (Fermi) velocity $v_{F}$ of a nucleon at the top of this Fermi. sea. Semi-classical means that the particles in this sea have short wavelengths compared to the size $R$.

It is especialily the first and last of these assumptions that drastically cut down the great richness of microscopic nuclear physics. However, the resulting idealized structure is not without $I$ ts own sharm and 1 s also useful, as $I$ hope to show.

The Degrees of Freedom of Leptodermous Nucle1

The leptoderwous Idealization follows from the saturation property of nuclear matter and the short-range nature of nuclear forces. It implies that a shape of a nuclear sustem may be defined (say, by the half-density contour of the density distribution). In fact, one of the major aims of macroscopic nuclear physics is to provide a theoretical description of the time evolution of a nuclear shape in processes such as fisston or nucleus-nucleus collisions. The shape defines the degrees of freediom of this dynamical problem. In order to make a theory of the process we need, as usual, three ingredients to put into Newton's equation of motion or into a Schrödinger equation:

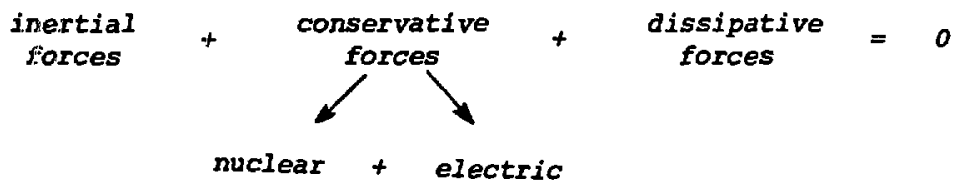


The conservative forces follow from a Potential Energy Function, so the first problem is to discuss the potential energy of a leptodermous nucleus as a function of its shape. This is the topic of the first lecture.

The Potential Energy of a Leptodermous Nucleus

The problem before us is to write down an expression for the potential energy of a diffuse blob of a certain shape $\Sigma$ as a functional of this shape. The blob may be in the form of one or more diffuse pieces, but the contour $\Sigma$ itself is, by definition, a sharpiy defined figure. The diffuseness of the surface of the blob is specified by the width $b$, of the order of the range of nuclear forces. The size of the blob is specified by a ra'jus $R$ (or volume $4 \pi R^{3} / 3$ ). We keep at the back of our minds the fact that the bl, $\Sigma$ is made up of elements that can feel each other over finite distances (of orct b) and that inside the blob $\Sigma$ there are wave functions that can feel out the shi pe of the whole biob, cver distances of the order of R (see Fig. 1). What will th- functional PE[ $\Sigma]$ look like in general?

Our experience with the nuclear problem has shown that it is useful to regard the total PE as made up of three parts, which I will refer to as a Local part, a Proximity part with a non-locality of range $\sim b$, and a Global part with a nonlocality of range $\sim R$.

$$
\begin{aligned}
\operatorname{PE}[\text { shape }] & =\text { Local Part } \\
& + \text { Proximity Part } \\
& + \text { Global Part } \\
& \approx \text { Analytic power series in (b/R) } \\
& + \text { Proximity Potential (non-analytic in } b / R \text { ) } \\
& + \text { Shell Effects (and the Coulomb Energy) }
\end{aligned}
$$

The Local Part is made up of contributions from different points in space. each contribution being a function only of the local conditions at the given point. The Proximity Part is made up of contributions that know about conditions a finite distance (of order b) away from the point in question. The Global Part cannot be written as a sum of local contributions - it knows about the shape as a whole.

In less formal language the local part would be called the liquid drop or droplet formula for nuclear energies (apart from the Coulomo energy). The Proximi.ty Part or Proxinity Potential shows up in the attraction (of range $\sim b$ ) between nuclear suriaces. The last part shows up as shell effects. (The Coulomb energy is actua :ly also an example of a global contribution.) Before I go into the derivation of sone approximate formulae for these contributions let me quickly remind you of their magnitudes and relevance in the nuclear context.

Figure 2 shows the mass decrements (essentially nuclear binding energies) of nuclei along the valley of beta stability. The absolute values of the nuclear binding energies are hundreds of $\mathrm{MeV}$. The smooth curve is a liguid-drop fit, i.e., a fit using the local part of the PE functional plus the Coulomb energy.

The curve in Fig. 3 is a plot of a theoretical expression for the interaction energy between nuclear surfaces as a function of their separation. The dots represent values deduced from elastic scattering and fusion data. The potential depthsi in this figure range to some tens of $\mathrm{MeV}$ and this illustrates the importance of the b-nonlocal contribution to the energy expression.

Figure 4 illustrates the magnitude of shell effects throughout the periodic table. The first line in each part of the figure gives the difference left over when a smooth liguld-drop part is subtracted from measured nuclear masses. The 
remaining oscillations are a few $\mathrm{MeV}$ (up to about $10 \mathrm{MeV}$ ) in magnitude and indicate the Importance of the global contributions that know about the whole shape and size of the nucleus.

I will come back to these figures later. We now come to the Leptodermous Potential Energy Theoren which states that the appearance of the local part is, under fairly general assumptions, of the following form [1]:

Relative Order

$$
\begin{aligned}
& P E \approx c_{1} \cdot(4 / 3) \pi R^{3} \quad \text { Volume energy } \\
& 1 \quad A \\
& +c_{2} \cdot \oint d \sigma \quad \text { Surface energy } \\
& +c_{3} \cdot \oint k d \sigma \quad \text { Curvature energy } \quad(b / R)^{2} \quad A^{1 / 3} \\
& \begin{array}{l}
+c_{b} \cdot \oint \Gamma \mathrm{d} \sigma \\
+c_{4}^{\prime} \cdot \oint k^{*} \cdot J
\end{array}\left\{\begin{array}{c}
\text { Higher-order curvature } \\
\text { corrections }
\end{array}\right\}(\mathrm{b} / \mathrm{R})^{3} \mathrm{~A}^{\circ} \\
& + \text { corrections that go to zero as } A \rightarrow \infty
\end{aligned}
$$

In the above,

$$
\begin{aligned}
K & =\text { total curvature at a point on the surface } \Sigma \\
& =1 / R_{1}+1 / R_{2}, \\
\Gamma & =\text { Gaussian curvature } 1 / R_{1} R_{2},
\end{aligned}
$$

where $R_{1}$ and $R_{2}$ are the principal radil of curvature at a point on $\Sigma$. The integrals are surface integrals over $\Sigma$. The coefficients $c_{1} \ldots c_{4}^{\prime}$ are constants independent of the shape and size of the system but may be functions of the bulk density.

You will soon get a feeling for the generality of this expression (and for its limitations) from the following

\section{Outline of Proof}

For a leptodermous system the density $\rho$ will look like the graph in Fig. $5 a$. One may also define an energy density $n$ which will look like Fig. 5b. For example, for a system of $A$ fermions described by an antisymmetrical wavefunction, $\psi\left(r_{1}, \ldots, r_{A}\right)$, one may define

$$
\begin{aligned}
& \rho\left(r_{1}\right) \equiv A \int_{r_{2}} \cdots \int_{\mathbf{r}_{A}} \psi^{*} \psi \\
& n\left(r_{2}\right) \equiv \int_{r_{2}} \cdots \int_{r_{A}} \psi^{*} H \psi,
\end{aligned}
$$

where $\mathrm{H}$ is the Hamiltonian of the A-particle system.

You can readily verify that the total number of particles is

$$
A=\iiint_{\rho}
$$




$$
E=\iiint n
$$

Starting with Eq. (7), add and subtract the number of particles times the energy per particle in the bulk (given by $a=n_{b u 1 k} / \rho_{b u l k}$ ):

$$
\begin{aligned}
E & =a A+\iiint(n-a \rho) \\
& =a A+\oint_{\Sigma} \int_{n}(n-a \rho) \\
& =a A+\oint_{\Sigma} Y
\end{aligned}
$$

We have split the triple integral over all space into an integral over the surface $\Sigma$ and an integral along the normal $\mathrm{n}$. This is useful since the integrand $n$-ap is in the form of a bump localized in the vicinity of the diffuse surface region (because $\rho, \eta$ tend to zero outside the surface region, and $n$ and ap cancel in the bulk - see Fig. 5c). We have denoted the result of the normal integration by $\gamma$. For a flat, semi-infinite density distribution this would be just the surface energy per unit area associated with a given surface. (This is because $\int \eta$ is the actual energy in a cylinder of unit area nomal to the surface, Ja $\rho$ is the energy that the same number of particles would have if they were in the bulk, so the difference is the surface energy.) For the actual shape $\Sigma$ that we are considering, the quantity $\gamma$ may, in general, be a functional $\gamma[\Sigma]$ of the whole shape. We now imagine $\gamma$ split up into a local and a non-local part, the local part being, by hypothesis, a function only of the local properties of $\Sigma$ at the poin: in question.

Now the local properties of a surface $\Sigma$ at some point on the surface are described, to lowest order, by the principal curvatures $1 / R_{1}, 1 / R_{2}$ at the point in question or, equivalently, by the invariant curvatures $K$ and $\Gamma$ defined earlier [Eqs. (2), (3)]. The dimensions of $K$ and $\Gamma$ are (length) ${ }^{-1}$ and (length) ${ }^{-2}$, respectively, so that the dimensionless arguments of which the local surface-energy coefficient $\gamma_{10 c} c a n$ be a function are $b k, b^{2} \Gamma$ (and higher-order inflection invariants that we need not consider). For a gently curved surface, for which $b$ is much smaller than the radil of curvature, we may expand as follows:

$$
\gamma_{1 o c}\left(b k, b^{2} \Gamma\right)=\gamma_{0}+\gamma_{K} k+\gamma_{\Gamma} \Gamma+\frac{1}{2} \gamma_{K K} k^{2}+\cdots,
$$

where $\gamma_{0}$ is the surface-energy coefficient for a flat surface and $\gamma_{K}, \gamma_{\Gamma}, \gamma_{K K}$ are the derivatives of this quantity with respect to $K, \Gamma$, evaluated for a flat surface. The quantities $\gamma_{0}, \gamma_{K}, \gamma_{\Gamma}, \gamma_{K K}$ are thus constants of relative order $1, b, b^{2}, b^{2}$ respectively.

Inserting $\mathrm{Eq}$. (9) Into Eq. (8) we obtain the Leptodermous Potential Energy Theorem. With respect to the leading volume-energy term the constants $c_{1} \ldots c_{4}^{1}$ are of the relative order $1, b_{b} b^{2}, b^{3}, b^{3}$, which Impiles energy contributions of order $A, A^{2 / 3}, A^{1 / 3}, A^{0}, A^{6}$.

Scope of the Theorem

Note the generality of this theorem, expected to apply to all kinds of thinskinned systems. Note that the crucial assumption is the locality of $\gamma$ (and not that the system is classical or that the mean free paths are short, as in a drop of water). But how good is the locality assumption for a system like a nucleus where the particles have long mean free paths and are approximated by indefendent- 
particle wave functions in a potential well? How large are the deviations caused by non-local effects? This will be illustrated by the discussion of the b-nonlocal and global contributions, especially of the shell effects which follow from the latter. But first let us look at the b-nonlocal part as given by the

\section{Proximity Potential Theorem}

The theorem states that the interaction potential berween two curved diffuse surfaces is given by [1]:

$$
V_{p}(s)=4 \pi \bar{R} \gamma b \Phi(s / b)
$$

Here $s$ is the least separation between the two surfaces (say between the two half-density contours), $\gamma$ and $b$ are, as before, the surface energy and width of the diffuse surface. The quantity $\bar{R}$ is a measure of the curvature of the two surfaces, which for two spheres with radil $C_{1}, C_{2}$ is given by the quantity

$$
\bar{R}=c_{1} c_{2} /\left(c_{1}+c_{2}\right)
$$

This is the "reduced radius" of the system. The quantity $\Phi$ is a dimensionless universal function of the dimensionless separation $s / b$. In the case of nuclei $\Phi$ may be approximated by the cubic-exponential formula

$$
\begin{aligned}
& \Phi\left(\zeta<\zeta_{1}\right)=-\frac{1}{2}\left(\zeta-\zeta_{0}\right)^{2}-k\left(\zeta-\zeta_{0}\right)^{3} \\
& \Phi\left(\zeta>\zeta_{1}\right)=-3.437 \exp (-\zeta / 0.75)
\end{aligned}
$$

where $\zeta_{1}=1.2511 \approx 5 / 4, \zeta_{0}=2.54 \approx$ inch $/ \mathrm{cm}, k=0.0852 \approx 1 / 12 \mathrm{a}$ Inch/foot. The function $\Phi$ is shown in Fig. 6 .

The derivation of the Proximity Potential is very simple and is based on replacing the interaction energy of two curved surfaces by a sum of contributions from parallel juxtaposed surface elements

$$
v_{\mathrm{P}} \approx \iint_{\text {gap }} \mathrm{e}(\mathrm{D}) \mathrm{d} \sigma
$$

The integral is over the transverse dinensions $x, y$ of the narrow gap between the surfaces and each contribution is approximated by $e(D) d \sigma$, the value $1 t$ would have if the surfaces were flat, parallel, and at the separation $D$ in question. (Thus each contribution to the potential energy depends on two finitely separated elements of the surface - hence the b-nonlocality.) The gap width between curved surfaces is a function of $x, y$. Expand $D(x, y)$ about the point of least gap width:

$$
D(x, y)=D(0,0)+\frac{1}{2} D_{x x} x^{2}+\frac{1}{2} D_{y y} y^{2}+\ldots \approx s+\rho^{2},
$$

where $D_{x x}, D_{y y}$ are the second derivatives of $D$ evaluated at $x=y=0$, and $\rho^{2}$ stands for $\left(\sqrt{D_{x x} / 2} x\right)^{2}+\left(\sqrt{D_{y y} / 2} y\right)^{2}$. Then we may re-write $V_{p}$ as

$$
v_{P}=\frac{2}{D_{x x y y} D_{y y}} \int_{0}^{\infty} 2 \pi \rho d \rho e(D)
$$

Slnce $2 \rho d \rho=d D$ from Eq. (14), we obtain, on changing variables from $\rho$ to $D$,

$$
v_{P}=2 \pi \bar{R} \int_{S}^{\infty} \mathrm{dDe}(D)=4 \pi \bar{R} \gamma \bar{D} \phi(s / b) \text {, }
$$


where $\bar{R}$ stands for $2 / D_{x x} D_{y y}$ and $\Phi$ is the dimensionless integral of $e(D)$ defined by

$$
\Phi(B / b)=\int_{B / b}^{\infty} d\left(\frac{D}{b}\right) \frac{e(D)}{2 \gamma}
$$

The function $e(D)$ (the interaction energy per untt area of two parallel surfaces at a separation $D$ ) is zero for $D \gg b$ and becomes equal to about $-2 \gamma$ at $D=0$, when the two juxtaposed diffuse densities have approximately added up to a uniform distribution at the standard equilibrium value of the matter in question and two units of surface area have been destroyed. On decreasing $D$ below zero the function $e(D)$ increases towards positive values as the two density distributions pile up. It follows that, at $D=0$, the function $e, " \gamma$ is about -1 and approximately stationary.

Extensions of the Proximity Theorem to other gap geometries and to crevices (formed by the non-overlapping portions of overlapping surfaces) are described in Ref. [1]. Thus, for a crevice formed by two slightly overlapping spheres with radii $R_{1}, R_{2}$, the proximity energy turns out to be (approximately) independent of the degree of overlap and (for small overlaps) to be given by

$$
\mathrm{V}_{\mathrm{P}} \approx 4 \pi \overline{\mathrm{R}} \gamma \mathrm{b} \Phi(0)
$$

It follows that for nuclei whose nuclear energy is estimated as the sum of their surface energies and a Proximity correction, the total potential for a sequence of configurations that includes a gap for $s>0$ and a crevice for $s<0$ is given by

$$
E_{\text {nuclear }} \approx 4 \pi \gamma\left(R_{1}^{2}+R_{2}^{2}\right)+ \begin{cases}4 \pi \bar{R} \gamma b \Phi(s / b) & \text { for } s>0 \\ 4 \pi \bar{R} \gamma b \Phi(0)+4 \pi \bar{R} \gamma s & \text { for } s<0 .\end{cases}
$$

The last term in Eq. (15c) represents the saving in the surface energy associated with the missing tips of the overlapping spheres. A simple straight-1ines approximation to Eqs. $(15 \mathrm{~b}, 15 \mathrm{c})$ wouid be

$$
E_{\text {nuclear }} \approx 4 \pi \gamma\left(R_{1}^{2}+R_{2}^{2}\right)+ \begin{cases}0 & \text { for } s>s_{1} \\ 4 \pi \bar{R} \gamma\left(s-s_{1}\right) & \text { for } s<s_{1},\end{cases}
$$

where

$$
s_{1}=-b \Phi(0)=1.78 \mathrm{~b} \approx \sqrt{3} \mathrm{~b}
$$

(The reason for writing $1.78 \approx \sqrt{3}$ will appear later.) A simple interpretation of Eqs. (15d,15e) is that the nuclear energy of the two spheres is to be estimated as proportional to the area of those parts of their surfaces which are separated by more than the distance $s_{1}$. (The last term in $\mathrm{Eq}$. (15e) represents the saving in the surface energy associated with the caps that are separated by less than $s_{1}$ and could be "Nitten as $-2 \pi r^{2}$, where $n$ is the radius of the caps.) The distance $s_{1} \approx \sqrt{3} b$ thus plays the role of an effective critical range of interaction such that, as a rough approximation, elements of the surfaces within this range may be assigned zero surface energy and elements outilde this range the full surface energy. We shall find this interpretation useful in Lecture III.

The Proximity Potential is being applied to many analyses of nuclear collisions and appears to give reasonable estimates for the interaction energy between nuclei. Really unambiguous tests of the Proximity expressions against experiment are not: easy because the Proximity theory is a theory of the interaction between nuclear surfaces as a function of their separation, but the precise locations of the 
relevant portions of the surfaces of two nuclel during a collision are, in genera1, not known. If the nuclel are assumed not to deform during the collision, then the surface separation can be deduced from the separation between the mass centers if the nuclear radil are sufficiently well known. There is mounting evidence that if such an undeformability assumption is made one gets the impression that the theoretical potential is of ten too weak by up to a factor of two. But it may well be that the calculated potential is in fact approximately correct but that tie surfaces have deformed, i.t., reached out towards each other by a few tenths of a fermi and thus increased the interaction at the given center separation of the two nuclei. Such polarization or deformation effects are to be expected and there is evidence ror them from other types of data. To follow up these hints quantitatively would be very instructive $[2]$.

Figure 3 gives a ruugh indication of the relation between estimated potential depths and the theoretical predictions. One might summarize by saying that a semiquantitative understanding of the b-nonlocal part of the nuclear potential energy has been achieved.

Let us now look at the third part of the energy, the global part that knows about the whole shape, namely, shell effects.

Shell Correction to the Potential Energy

Let us return to the question: how good should one expect the local approximation to be for a quantal system like a nucleus, consisting of long-path (dolichohodous) particles, approximated by quantized independent-particle wavefunctions in a potential well? There have been many studies of this problem [6,7]. I will use some examples from an unpublished thesis by Chin-Fu Tsang to illuminate the situation $[3,4]$.

Figure 7 shows a portion of a Nilsson level diagram of independent quantized nucleons in a modified oscillator potential [5]. As you know, such a Nilsson diagram has been at the heart of theories of nuclear structure for many years. In the last decade it has also been used in the Strutinsky method for estimating shell corrections [6]. In this method, one takes a sum over the eigenvalues displayed in Fig. 7 and subtracts a smooth background, representing the liquid drop or local part of the energy. The essence of the problem is not changed, but the aspects relevant to our discussion are much easier to disentangle if, instead of a modified oscillator potential well, one takes the much simpler Hill-wheeler potential well. This well is in the form of a box with sides $a, b, c$, the potential being zero inside and infintte outside the box. The eigensolutions are trivial products of sines and the elgenvalues are sums of squares of positive integers. A "Nilsson diagram" for this box looks like Fig. 8. Here the efgenvalues are again plotted as a function of deformation. The axis at $a=0$ corresponds to a cubical box with $a=b=c$ and on the right are prolate shapes with $a=b<c$ and on the left oblate shapes with $a=b>c$. Anyway, here is a simple type of Nilsson diagram for which we can easily calculate the sum over eigenvalues

$$
E=\sum_{i=1}^{A} E_{i}
$$

and compare this with a smooth power series expansion representing the local part of the energy. (In the case of the Hill-Wheeler box the potential well has zero diffuseness. Nevertheless, the density distribution of the particles filling such a well has a finite diffuseness, of order of the wavelength, $\lambda_{F}$, of a particle at the top of the Fermi sea. The small dimensionless parameter in the local series expansion is $\hbar_{F} / R \sim b / R$, as before.) 
Figure 9 compares the sum over eigenvalues for a cublc box (shown as a function of the number $N$ of filled elgenstates, with $N=A / 4$ ). The local part of the energy expression was calculated to different orders in $N$ (or $A$ ). The straight line labeled $E^{\prime}(N)$ is the volume energy proportional to $N$, the next curve up includes the surface energy proportional to $\mathrm{N}^{2 / 3}$ and the uppermost curve includes the term in $\mathrm{N}^{1 / 3}$. The exact sum over eigenvalues falls on top of this curve and cannot be distinguished from it on the scale of Fig. 9. (The units on the ordinate are roughly of the order of $\mathrm{MeV}$.)

Figure 10 shows the difference bitween the sum over eigenvalues and the local part taken to order $\mathrm{N}^{2} / 3$ (upper curve) and to order $\mathrm{N}^{1 / 3}$ (lower curve). The scalloped appearance of the sum over eigenvalues is due to shell effects (e.g., $\mathrm{N}=60$ is a closed shell for a cubic box). Most of the deviation associated with the upper curve is, indeed, removed by inciuding the $\mathrm{N}^{1 / 3}$ term in the local expansion. The remaining deviations a sear to be of the order of several $\mathrm{MeV}$ and to consist of an oscillating shell correction and a constant term (i.e., a term of order $\mathrm{N}^{\circ}$, as expected from the lozal power expansion). That this is really the case is illustrated by Fig. 11 where the lower curve from Fig. 10 is now extended to $N=1500$ (i.e., a system with $A=6000$ ). It would appear that in this case the global (shell-correction) part of the energy is of the order of a few $\mathrm{MeV}$ and its amplitude appears to be independent of $\mathrm{A}$.

Our understanding of shell effects, especially of their relation to the symetries of the potential weli and to properties of classical orbits in the sarue well, has advanced considerably in the past few years [see Refs. 6,7]. From such considerations the A-dependence of shell effects in a cubic box is expected to be $A^{\circ}$, but may be different when other svinmetries are present. The empirical shell effects (see Fig. 4) are not inconsistent with an $A^{\circ}$ dependence, but the range in $A$ is too limited for this to be more than a rough estimate.

Figure 12 shows the deviation of the sum over eigenvalues from the local expansion taken to order $\mathrm{N}^{2 / 3}$ (lower curve) or $\mathrm{N}^{1 / 3}$ (upper curve). This time the value of $\mathrm{N}$ is fixed $(\mathrm{N}=60)$ but the shape of the box varies from a cube (at $\alpha=0$ ) to a prolate box with $a / c=b / c=0.4724$ at $\omega=0.5$. Most of the deviation from the $\mathrm{N}^{1 / 3}$ curve could be accounted for by a small term of order $\mathrm{N}^{\circ}$.

These and similar but more extensive studies in Ref. [3], as well as other calculations in different potentials, confirm the usefulness and relative accuracy (down to the level of a $f(e w \mathrm{MeV}$ ) of the local power expansions in $b / R$, even for strictly quantal particles with long mean free paths.

This was not originally expected (and is even today not widely recognized) because the basic reason is quite subtle. It has to do with the fact that, although each eigenvalue and eigenfunction is totally "global" (i.e., it knows about the whole shape of the well) and the energy density associated with a single wave function is certainly not leptodermous, the energy density of an assembly of particles of ten acquires th? leptodermous character of a uniforw bulk energy density modifled by a contribution essentially confined to the surface region. This has to do with a randomization of the relative phases of the wavefunctions away from the surface region.

For a quantitative treatment of the MeV-rype deviations associated with shell effects we have avallable today the Strutinsky shell correction method, which I mentioned earlier. Its effectiveness is illustrated in the lower part of Fig. 4. There, the second line, labeled (b), displays a theoretical she1! correction obtained using this method. Line (c) shows the remaining deviations. The 'Ipper half of Fig. 4 is similar, but the theoretical shell correction was obtained using a schematic spectrum of eigenvalues rather than the one based on the Nilsson level scheme. The remaining deviations are again shown in the last ine. 
In this lecture we considered the problem of writing down the Pctential Energy of a nuclear system as a function of 1ts shape, PE[ $[\Sigma]$. From general arguments we anticipated that there might be three parts in the potential energy expression: a local part, a non-1ocal part with a range of the order of the range of nuclear forces, and a global part. We derived an approximate expression for the local part, in the form of the Leptodermous Expansion in powers of $b / R$, and for the $b$-nonlocal part in the form of the Proximity Energy. The local part may be many hundreds of $\mathrm{MeV}$ in magnitude, the Proximity potential may be a few tens of MeV. After adiirs calculated shell effects, which are generally a few MeV, we have available today a pretty good estimate of the nuclear potentlal energy and thus also of the conservative driving forces that will govern nuclear dynamical motions.

In the next lecture we shall consider the frictional resistance to these driving forces. A key ingredient in that analysis will again be the leptodermous and the local idealizations, according to which much of the Interesting physics is localized in the surface region.

\section{LECTURE II}

The first lecture was concerned with the macroscopic nuclear Potential Energy Function, from which the conservative driving forces can be derived. The present lecture will deal with the macroscoplc nuclear Energy Dissipation Function from which the dissipative frictional forces opposing these driving forces can be deduced.

The treatment I will describe goes by the name of the "One-Body Dissipation Theory" (since the one-body, Independent-particle aspect of nuclear structure is an essential assumption). I am very fond of this theory, principally because it is so utterly simple, but it is fair to point out at once that there has been and still is a lot of skepticism as to the validity and scope of this approach. I hope our discussions at this School may throw some more 1ight on this problem.

\section{Background}

What led to the One-Body Dissipation theory was the simple question: is nuclear dynamics dominated by dissipation or not? Are nuclel more like honey or like water? It is strange that after half a century of studying nuclei we don't really know for certaln the answer to such a basic question.

The One-Bcdy Dissipation theory attempts to answer this question by making use of the approximate validity of the long-mean-free path, mean-fleld aspect of nuclear structure. This is, of course, also the starting point of the sheli model, the Time-Dependent Hartree-Fock theory, and of nuclear linear-response theorles.

The physics of the one-body dissipation is the irreversible flow of energy from the collective to the particle degrees of freedom, resulting from the collisions of the lang-mean-free-path particles with the moving boundary of the nuclear potential well. (The flow is irreversible under a certaln Randomization Hypothesis.)

By contrast, in the case of the dissipative flow of ordinary fluids governed by ordinary viscosity, the irreversible flow of energy is caused by (two-body) collisions between the molecules, whose mean-free paths $\mathrm{L}$ are (almost always) short compared to the size $R$ of the system. Thus we have 


$\begin{array}{lll}\text { One-body dissipation } & \text { if } & L \\ \text { Two-body dissipation } & \text { if } & L \ll R\end{array}$

The Wall and the Window Formulae

Very simple formulae for the one-body dissipation follow in three situations:

a) For a freely communicating (1.e., not necked-in) shape w? shall find the Wall Formula.

b) For shapes in the form of two weakly communicating rigid pleces in relative motion we shall find the window Formula.

c) An obvious generalization of (b) to the case when the two pieces are deformable is the Wall-plus-Window Formula.

Let me write down the wall and window formulae and sketch their derivations.

The Wal1 Formula

Imagine a container filled with a dolichohodous gas, as in Fig. 13. The bulk of the gas is assumed to be at rest (i.e., there are no overall translations or rotations present). Let the mass density of the gas be $\rho$, the mean speed of the particles be $\bar{v}$, and imagine that the container is undergoing a deformation (at fixed volume) specified by normal velocities $\dot{n}$ of its surface elevents do. Then the Wall Formula states that, if the Randomization Hypothesis is satisfied, the irreversible flow of energy from the wall degrees of freedom into the particle degrees of freedom is given by

$$
\dot{\mathrm{Q}} \equiv \frac{\mathrm{dE}}{\mathrm{dt}}=\rho \overline{\mathrm{v}} \oint \dot{\mathrm{n}}^{2} \mathrm{~d} \sigma \quad,
$$

where the integration is over the surface of the vessel.

The physics of the wall formula is illustrated by considering the case of a piston moving with speed $\dot{n}$ away from a long-mean-free-path gas in an (infinitely) long cylinder. A trivial application of the kinetic theory of gases shows that the pressure on the piston is given by

$$
p=\frac{1}{3} \rho \overline{v^{2}}-\rho \bar{v} \dot{n}+\text { higher powers of } \dot{n} / \bar{v} \text {. }
$$

The first term is the standard result which leads to the ideal gas law ( $\mathrm{pV}=\mathrm{NRT}$ ). The next term is a correction for the motion of the piston. Ii expresses the reasonable expectation that for a piston receding from the gas the pressure is a little lower, and for a piston moving into the gas the pressure is a little higher than the standard value $(1 / 3) \rho v^{2}$. I will not go through the trivial derivation of this correction, but merely noce that the only physics involved is the kinematics of the elastic collision of a particle with a moving wall. On integrating the pressure in Eq. (17) (nultiplied by the displacement $\delta$ of a surface element) over the surface of the deforming vessel, one obtains the work done by the vessel on the gas. On dividing the result by the time element $\delta t$, one finds, for volumepreserving deformations, the rate of doing work given by Eq. (16).

The Randomization Hypothesis comes in at the stage when one applies the pressure expression, Eq. (17), derived for a piston moving with respect to an undisturbed (infinitely) long cylinder filled with a gas, to the individual surface elements of a finite container. Only if one is allowed to assume that, at each instant of time, the surface elements continue to be bombarded by the gas molecules as If these originated in the bulk of a gas at rest is one justiffed in using $p-\dot{n}^{2} \mathrm{~d} \sigma$ for the rate of dissipation. If the vessel has an irregular surface and is deforming in an irregular way, the Randomization Hypothesis may, indeed, be 
satisfied. But it is very easy to demonstrate that in many simple cases - in fact in cases that are most familiar and come to mind most readily - the Randomization Hypothesis is obviously not valid. These are cases where the vessel is characterized by symetries or regularities (e.g., a spherical or a cubic potential we11) or, even when the vessel is irregular, If the motion of the surface elements is characterized by regularities. The obvious case in the latter category is a uniform rotation or uniform translation of the vessel. Formula (16) would 1mply the absurd result that even for such steady motions there would be a continued transfer of energy from the walls to the particles.

The reason for this absurdity is clear: when the vessel is set into steady rotation or translation the gas is quickly made to co-translate and co-rotate. The surface elements are then bombarded by a gas that is not at rest but has a drift motion. When this motion has caught up with that of the vessel the pressure returns to the standard value $(1 / 3) \rho \overline{v^{2}}$ and there is no dissipation.

In order then to treat the case of a deforming vessel that may, in addition, be translating and rotating, one needs a generalized wall formula that takes into account the drifts set up in the gas by the translation and rotation. Such a formula is derived, under certain assumptions, in section 7 of Ref. [8]. Here I shall just state the result. The generalized wall formula reads as follows:

$$
\dot{\mathrm{Q}}=\rho \overline{\mathrm{v}} \oint(\dot{\mathrm{n}}-\mathrm{D})^{2} \mathrm{~d} \sigma \quad \text {, }
$$

where $D$ is the normal drift component of those gas particles about to strike the surface element do. This drift $D$ varies from point to point and is a function of the state of motion of the container. Specifically, it is a function of an effective translation $\vec{v}$ and an effective rotation $\vec{\Omega}$ about the center of mass of the container, given by

$$
D=(\vec{V}+\vec{\Omega} \times \vec{R}) \cdot \breve{n},
$$

where $\breve{n}$ is the normal to the surface at the point in question and $\vec{v}, \vec{\Omega}$ are the solutions of the following pair of linear vector equations:

$$
\begin{aligned}
& \{[\dot{n}-(\vec{V}+\vec{\Omega} \times \vec{R}) \cdot \ddot{n}] \breve{n} d \sigma=0 \\
& \oint(\vec{R} \times \grave{n})[\dot{n}-(\vec{V}+\vec{\Omega} \times \vec{R}) \cdot \breve{n}] d \sigma=0 .
\end{aligned}
$$

Here $\vec{R}$ is the radius vector from the center of mass of the container to the surface element $d \sigma$. Equations $(20,21)$ define $\vec{V}, \vec{\Omega}$ in terms of the configuration of the vesse1 (given by $\vec{R}$ ) and its state of motion and deformation (given by $\dot{n}$ ). (The vectors $\vec{\nabla}, \vec{\Omega}$ are the "Instantaneous tracking parameters" of the container's motion.) Equations $(20,21)$ express the conservation of the total linear and angular momenta of the particles in the container and ensure that the generalized wall formula, Eq. (18), does not come into conflict with these conservation laws in the way that Eq. (16) did in the case of steady translations or rotations.

\section{The Window Formula}

Figure 14 illustrates two rigid containers in relative motion communicating through a small window of area $\Delta \sigma$. The relutive velocity between the two containers has radial and tangential components $u_{r}$ and $u_{t}$. The window formula states that the rate of dissipation of the energy of relative motion is given by

$$
\dot{Q}=\frac{1}{4} \bar{v} \Delta \sigma\left(u_{t}^{2}+2 u_{r}^{2}\right) \text {. }
$$


The quantity $\frac{1}{4} \rho \bar{v} \Delta \sigma$ is the one-sided flux of particles through the window and this mass flux flowing both ways between two objects whose velocities are mismatched results in a drag and an associated dissipation of energy. The physics of this dissipation is illustrated by the example of two coal trains coasting with different speeds along parallel tracks. If a crew of madmen furiously shoveled coal back and forth, the relative speed of the trains would be reduced by the drag between them, caused by the fluxes of coal. Similarly, if the brakes failed on two trains headed towards a collision, such shoveling, if vigorous enough, would avold a serious crash.

In the case of the Window Formula, the Randomization Hypothesis is hidden in the tacit assumption that particles which have crossed the window do not re-cross before they have acquired the drift characteristic of the receptor vessel. A completely analogous assumption is made in the derivation of the expression for the ordinary viscosity coefficient according to the kinetic theory of gases. There, particles leaving one layer of fluld are imagined to be deposited in another layer, at a distance of the order of the mean free path, whose velocity is mismatched. They are not supposed to come back to the original layer before they have acquired the drift of the receptor layer. (Incidentally, pseudo-profound questions of how reversible equations of motion can lead to irreversibility can be referred to this familiar and down-to-earth result of the kinetic theory of gases.)

\section{The Wall-Plus-Window Formula:}

This is a falrly obvious combination of the (generalized) wall and window formulae, relevant in the case when the weakly communicating vessels are deformable rather than $\mathbf{r} f$ idd. The energy dissipation may then be approximated by writing

$$
\dot{Q}=\frac{1}{4} \rho \bar{v} \Delta \sigma\left(u_{t}^{2}+2 u_{r}^{2}\right)+\rho \bar{v} \oint_{1}\left(\dot{n}-D_{1}\right)^{2} d \sigma+\rho \bar{v} \int_{2}\left(\dot{\mathrm{n}}-D_{2}\right)^{2} d \sigma,
$$

where the wall diseipation consists of two pieces, one for each fragment, in the form of $\mathrm{Eq}$. (18). The drifts $D_{1}$ and $D_{2}$ are to be related to the configurations and states of motion of the two pieces by expressions of the type of Eq5. (19-21). A simple application of $\mathrm{Eq}$. (23) will be presented in Lecture III.

\section{Obvious Questions}

Here is a partial list of questions that come to mind when one is tempted to apply the simple formulae derived above to nuclear systems. First, what about quantization?

1. How will the wave-mechanical nature of the particles affect the wall and window formulae?

2. What effect will the discreteness of the eigenvalues have on the results?

3. Will the pauli exclusion principle change the results, perhaps drastica1ly?

Second, even for a classical assembly of particles, what about the

4. Randomization Hypothesis? How good is it in various situations?

5. What about self-consistency, 1.e., the fact that a nuclear potential well is not an external vessel filled with particles but an object generated by and tied to the particles?

6. How good is the generalized wall formula with drifts? In a case like fission, how does one make a gradual transition from the wall formula 
to the wa11-plus-window formula as the communication between the two pieces becomes impeded by the decreasing neck?

These questions have been discussed to a varying extent in several papers in the past few years [see, for example, Refs. 8,9]. Some of them will be iliumlnated by the leptodermous theorem for nuclear dissipation that I am about to describe. To others I hope to come back at the end.

\section{Leptodermous Theorem for Nuclear Dissipation}

This theorem is analogous to the Leptodermous Potential Energy Theorem in Lecture I. It states that, under certain assumptions, the local part of the nuclear One-Body Dissipation Function can be written in the following form:

$$
\begin{aligned}
& \dot{Q}=k_{1} \text { (volume integral) } \\
& +k_{2} \oint \dot{n}^{2} d \sigma \quad \text { Wall formula } \quad \text { i } A^{2 / 3} \\
& +k_{3} \oint \dot{n}^{2} k d \sigma \quad \text { Curvature correction } b / R \quad A^{1 / 3}
\end{aligned}
$$

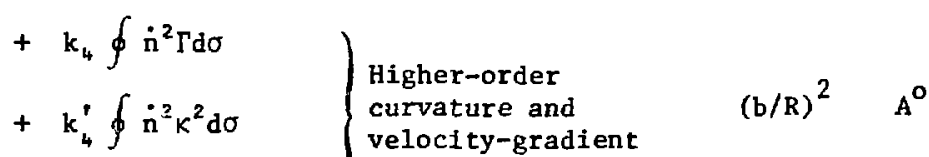

$$
\begin{aligned}
& \left.+k_{4}^{\prime \prime} \oint(\operatorname{grad} \dot{\mathrm{n}})^{2} \mathrm{do}\right) \text { corrections } \\
& + \text { corrections that go to zero as } A+\infty \text {. } A^{-n}
\end{aligned}
$$

The coefficients $k_{1} \ldots k_{4}^{\prime \prime}$ are again constants (independent of shape and size, but dependent, in general, on the bulk density). The quantity (grad $\dot{n}$ ) stands for the two-dimensional gradient of the velocity field $\dot{n}$ considered as a function of position on the surface $\Sigma$.

\section{Sketch of Proof}

The derivation goes like this: We are again given a diffuse leptodermous blob whose shape is defined by a sharp figure $\Sigma$, but this time the shape is a function of time, which I will denote by $\Sigma(t)$. The diffuse surface has a widtk $b$ as before, and the blob is regarded as made up of long-mean-free-path particles. The general question is: what is the appearance of the One-Body Dissipation Functional $\dot{Q}[\Sigma(t)]$ ? The theorem I have written down gives the answer under the assumption of locality, as in the case of the Potential Energy. The essence of the derfvation is that since the system 1 s supposed to be leptodermous and the mean free paths long, the particles travel in straight lines (and become approximately plane waves if quantized) except when they enter the diffuse surface region. It follows that even when the system is in a state of deformation the energy of eack particle is a constant of the motion except when it enters the diffuse surface. As a result, any interchange of energy between the shape degrees of freedom and the particle degrees of freedom can take place only in the surface region. This simple observation has far-reaching consequences.

Thus it should be possible to write the rate of one-body dissipation in the following form: 


$$
\dot{\mathbf{Q}}=\iiint \dot{q} \quad
$$

where the integrand $\dot{q}$ is confined to the surface region. Because this "dissipation bump function" $\dot{q}$ is formally similar to the "surface energy bump function" $(n-a p)$ from lecture $I$, it suddenly dawns on one that it is possible to make exactly the same leptodermous expansion $i n b / R$ as for the potential energy.

Thus we re-write Eq. (25) as

$$
\begin{aligned}
\dot{Q} & =\oint_{\Sigma} \int_{\mathrm{n}} \dot{q} \\
& =\oint_{\Sigma} \mathrm{Y} d \sigma,
\end{aligned}
$$

where $Y$ stands for the result of integrating over $\dot{q}$ along the normal $\mathfrak{n}$. This quantity $Y$ is a "specific rate of dissipation per unit area of a moving surface," analogous to $\gamma$, the specific surface energy per unit area. For a perfectly flat surface of a semi-infinite system, $i . e .$, for $b / R \rightarrow 0, Y$ reduces to $Y_{O}$, the result given by the wall formula:

$$
\mathrm{Y}_{\mathrm{o}}=\rho \overrightarrow{\mathrm{v}}^{2} \quad \text {. }
$$

In the case of a finite system, $Y$ may, in general, be a functional $Y[\Sigma(t)]$ of the whole shape $\Sigma(t)$, 1ncluding its time history. We now imagine $Y$ split up into a local part, $Y(\Sigma, \Sigma)$, which is a function of the local properties of $\Sigma$ and its rate of deformation $\Sigma$ (evaluated at a point on the surface and at an instant of time) and the remainder, which may be non-local.

By the same arguments as before, Ylocal can be a function of the local invariants $\kappa, \Gamma, \ldots$ at a point (more precisely of the dimensionless arguments $\left.\mathrm{bK}, \mathrm{b}^{2} \Gamma, \ldots\right)$. In addition, $\mathrm{Y}_{\text {local }}$ can be a function of the local kinematic invariants of the velocity field $\dot{n}$, consldered as a function of position on the surface $\Sigma$. The lowest-order kinematic invariant turns out to be $\left(\mathrm{grad}^{\mathrm{n}}\right)^{2}$, the square of the two-dimensional gradient of this function $\dot{n}$. The relevant dimensionless quantity of which $Y$ can be a function is then $b^{2}(\operatorname{grad} \dot{n} / \bar{v})^{2}$. The function that we have to consider is thus

$$
\left.Y_{\text {local }}=Y_{i b k}, b^{2} \Gamma, \ldots ; b^{2}(\operatorname{grad} \dot{n} / \bar{v})^{2}, \ldots\right) .
$$

Expanding $y$ in powers of $1 t s$ arguments and retaining terms up to $b^{2}$, one finds (after making use of sywmetry arguments and some transformations described in Ref. 9) that the surface Integral over Y local [Eq. (26)] has terms in $K, K^{2}, \Gamma$, ( $g \mathrm{rad} \dot{\mathrm{n}})^{2}$, which can be arranged according to powers of $b$ in the form of the Leptodermous Dissipation Theorem that I wrote down earlier [Eq. (24)].

The scheme is similar to the PE expansion except that there is no volune term. I did include a symbolic term $k_{1}$ (Volume Integral) as a reminder that in a less idealized theory (where the mean free paths are not infinitely long and the nuclear potential in the bulk is not dead flat and independent of time) one would expect a (sma11) contribution from the bulk. The pattern of terms in Eq. (24) follows the pattern in Eq. (1), except for the extra factors $\dot{n}^{2}$, and for the new term $\mathrm{k}_{4}(\mathrm{grad} \dot{\mathrm{n}})^{2}$, the "velocity-gradient correction." 
After decades of nuclear physics the first two coefficients in the Potential Energy Expansion, Eq. (1), are well known and the third is known approximacely [10]:

$$
\begin{aligned}
& c_{1}=2.32 \mathrm{MeV} \mathrm{fm}^{-3} \\
& c_{2}=1.2 \mathrm{MeV} \mathrm{fm}^{-2} \\
& c_{3} \approx 0 \\
& c_{4} \text { and higher unknown }
\end{aligned}
$$

In the case of the Dissipation Expansion we have $k_{1} \approx 0$, and $k_{2}=p \bar{v}[E q$. (27)]. Expressing the mass density and mean speed of a degenerate Fermi gas in terms of the radius constant $r_{0}$ one finds

$$
\begin{aligned}
\rho \bar{v} & =\frac{27}{32 \pi}\left(\frac{\pi}{3}\right)^{1 / 3} \frac{\hbar}{r_{0}^{4}} \\
& =0.272735 \pi / r_{0}^{4} \\
& =1.026 \times 10^{-22} \mathrm{MeV} \mathrm{sec} \mathrm{fm}^{-4},
\end{aligned}
$$

if an 1llustrative value $r_{0}=1.15 \mathrm{fm}$ is used. Estimates of the curvature-correction coefficient $k_{9}$ have recently become avallable [9]. Thus using a semi-classical method applied to independent particles in a potential well with a linear potentlal profile in the surface one finds:

$$
\mathrm{k}_{3} / \mathrm{k}_{2}=\sqrt{28 / 27} \mathrm{~b} \approx 1.02 \mathrm{fm} .
$$

For a parabolic potential profile one finds:

$$
\mathrm{k}_{3} / \mathrm{k}_{2}=\frac{19}{45}\left[\frac{2}{5}\left(\frac{16}{3 \pi}\right)^{2}-1\right]^{-\frac{1}{2}} \mathrm{~b} \approx 1.08 \mathrm{fm}_{\mathrm{m}}
$$

A quantal calculation for a gas of independent particles, bounded by an infinitely steep potential well (not a realistic model of a nuclear surface) one finds

$$
\mathrm{k}_{3} / \mathrm{k}_{2}=\frac{3 \pi}{8 \mathrm{k}_{\mathrm{F}}} \approx 0.89 \mathrm{fm} .
$$

(Here $\mathrm{k}_{\mathrm{F}}$ is the wave-number of the particles at the top of the Fermi sean)

Estimates of the higher coefficients are not avallable.

We may sumbarize the estimates as follows

$$
\begin{aligned}
& k_{1} \approx 0 \\
& k_{2} \approx 1.0 \times 10^{-22} \mathrm{MeV} \sec \mathrm{fm}^{-4} \\
& k_{3} \approx 1.0 \times 10^{-22} \mathrm{MeV} \sec \mathrm{fm}^{-3} \\
& k_{4} \text { and higher unknown }
\end{aligned}
$$

Even though these estimates are to b. regarded as only rough (because of the Iimited accuracy of the approximations that have been made) it is worth noting that In discussing the dissipation function $i$ we are actually better of than in the 
curresponding discussion of the Potential Energy. There, the mere realization of the approximate validity of the independent-particle model does not enable one to make an estimate from first principles of the binding energy per particle of nuclear matter or of the surface and curvature-correction coefficients. In the case of the dissipation the approximate validity of the dolichohodous and leptodermous idealizations is sufficiently far-reaching to suggest not only the functional form of the local part of the dissipation function but also the approximate values of the relevant expansion coefficients.

In addition to the local part of $\dot{Q}[\Sigma(t)]$ one expects b-nonlocal and global terms, as in the Potential Energy. Limited studies of such effects are described in Refs. $[8,9]$, but much remains to be done, relatively more than in the case of the PE.

Taking the local part at face value, the most striking prediction of the theory is that the one-body ilssipation is so large in absolute magnitude that it might often dominate nuclear dynamics. By this I mean that (if and when the onebody dissipation is applicable) the dissipative forces nay be in fact more important than the inertial forces. In those cases a nucleus would be more 1ike a drop of honey than a drop of water.

The way to demonstrate this in an order-of-magnitude way is to compare the inertial and dissipative forces in a typical case or, what comes to the same thing, to estimate the time it would take a certain amount of collective Kinetic Energy $E$ to be dissipated by the one-body dissipation $\dot{Q}=-\mathrm{dE} / \mathrm{dt}$. Thus consider some (preferably irregular) nuclear shape, whose surface is started off in a ripple of multipole order $\ell$ and with a typical surface speed $\dot{n}$. Let us estimate the initial kinetic energy as one-half times a typical mass times the square of a typical velocity. For the typical mass let us take the mass of the nucleus, $(4 / 3) \pi R^{3} \rho$, divided by $\ell$ (since a multipole ripple divides the nucleus into about $\ell$ cells). The typical velocity will be of order $\dot{n}$. Hence, after dropping numerical factors of order one, we have

$$
E \approx R^{3} \rho \dot{n}^{2} / \ell .
$$

According to the Wall Formula the rate of energy dissipation is $\rho \bar{v}$ times the area of the nucleus times a rypical value of $\dot{i}^{2}$ :

$$
\begin{aligned}
-\frac{d E}{d t} & \approx \rho v\left(4 \pi R^{2}\right) \dot{n}^{2} \\
& \approx R^{2} \rho \bar{v} \dot{n}^{2}
\end{aligned}
$$

Hence the e-folding time to dissipate the kinetic energy is

$$
\mathrm{t}_{\text {diss }} \approx-\mathrm{E} / \dot{\mathrm{E}} \approx \frac{1}{\ell}\left(\frac{\mathrm{R}}{\overline{\mathrm{v}}}\right)
$$

The first factor is a geometrical quantity, which varies with the order $c$ f the ripple. The second factor is the characteristic time unit of the one-body dissipation theory. It is of the order of the single-particle transit time

$$
\mathrm{R} / \overline{\mathrm{v}} \approx 0.7-1.3 \times 10^{-22} \mathrm{sec}, \text { for } \mathrm{A}=50-250 \text {, }
$$

or

$$
\mathrm{h}(\overrightarrow{\mathrm{v}} / \mathrm{R}) \Rightarrow 5-9 \mathrm{MeV} \text {. }
$$

This sfinple result could have been anticipated on dimensional grounds since, With the mean free path assumed long, there is no other length than $R$ and no other speed than $\bar{v}$ in the underlying physics to make up a unit of time. 
Since this damping time is so short the implication is that very soon (after a time typically measured in a unit of $10^{-22} \mathrm{Bec}$ ) a large fraction of any kinetic energy originaliy present would be dissipated and the further time evolution of the system would proceed approxtmately according to a balance of conservative and dissipative forces, the inertisi forces becoming relatively small. (This is the familiar situation for overdemped systems, such as a drop of honey.)

In Lecture III we shall investigate further this type of dynamics. This is possible since we have now derived simple formilae for describing approximately the two principal ingredients: the Conservative Driving forces in Lecture 1 and the Dissipative forces in Lecture II. By putting them together we shall derive a prototype equation of motion for the dynamics dominated by one-body dissipation (the "New Dynamics"). Then we shall go through a number of applications that have been made in the nuclear context.

\section{LECTURE III}

In the first lecture we discussed the macroscopic Potential Energy, in the sacond the macroscopic One-Body Dissipation Function. In this lecture I will put the two components together to derive a very approximate but very simple equation of motion supposed to govern the gross features of the time evolution of a nuclear shape. Then I will discuss some qualitative and semi-quantitative applications to nuclear fission and nucleus-nucleus collisions.

Since this represents an early attemp to write down such an equation of motion I will treat the problem at the simplest possible level. The most drastic simplification will be the neglect of all shell effects. This may be a fair approximation to reality in some cases but is certainly grossly inaccurate in others. The less drastic approximation will be the truncation of the leptodermous expansions at the leading terms (both for the PE and the Dissipation Function).

In this bare-bones approximation the mathematical problem may be stated as follows: we are given an Idealized nucleus spexified by a shape $\Sigma$. The nucleus is incompressible, is electrically charged with a uniform charge density $\rho_{e}$ and is endowed with a surface energy per unit area $Y$. if the shape $\Sigma$ is in a state of defortacion, the rate of energy dissipation is assumed to be given by the wall formula [Eq. (16), Lecture II]. The use of Eq. (16) implies that we are further restricting the discussion to the case when there are no drifts and no constrictions in the shape $\Sigma$. The question is now: what is the equation of motion governing the time evolution of the shape $\Sigma$ ?

Wo answer this by considering an Infinitesimal deformation of $\Sigma$, specified by normal displacements $\delta$ of the surface elements do. We write down the change in the potential energy associated with this deformation as well as the dissipated energy. Equating the total energy change to zerc leads to the desired equation of motion under the assumption that the Kinetic Energy is negligible, 1.e., that inertfal forces are negligible. (This assumption will be tested presently and relaxed in some of the applications.)

The relevant potential energy is the sum of Coulomb and surface energies

$$
P E=\frac{1}{2} P_{e} \iiint \phi d^{3} x+\gamma \oint d \sigma \quad
$$

where $\phi$ is the electric potential. The change in energy associated with a deformation described by normal displacements on is (by elementary el srostatics and geometry, e.g. Ref. [11]): 


$$
\delta(P E)=\rho_{e} \oint \phi \delta n d \sigma+\gamma \oint k \delta n d \sigma
$$

where $\phi$ is the electric potential at a point on the surface and $k$ is the curvature of the surface at that point.

The energy dissipated during the deformation in question may be written as

$$
\delta E=\dot{\mathrm{Q}} \delta \mathrm{t}=\rho \overline{\mathrm{v}} \oint \dot{\mathrm{n}} \delta \mathrm{ndo} .
$$

If the Kinetic Energy is neglected, energy conservation demands that

$$
\oint\left(\rho \bar{v} \dot{n}+\rho_{e} \phi+\gamma K\right) \delta n d \sigma=0 .
$$

If Eq. (36) is to be satisfied for volume-preserving deformations we must have

$$
\rho \vec{v} \dot{n}+\rho_{e} \phi+\gamma k=\text { constant. }
$$

(This constant. may then be taken outside the integral sign in Eq. (36), and the remaining integral $\oint \delta$ ndo is the change in volume, which ic zero by hypothesis.)

The constant in Eq. (37) may be determined by subtracting from this equation its surface average. Since the surface average of $\dot{n}$ is zero, this gives

$$
\rho \ddot{v} \dot{n}-P=0,
$$

where

$$
P=\rho_{\mathrm{e}}(\bar{\phi}-\phi)+\gamma(\bar{x}-\kappa),
$$

with $\bar{\phi}$ the surface average of the surface potential and $\bar{k}$ the surface average of the curvature. Hence

$$
\frac{d n}{d t}=P / \rho \bar{v}
$$

This is the desired equation of mc:ion, specifying the displacements on of the surface in a time $\delta t$ in terms of quantities defined by the configuration in question. Equation (40) states that the rate of displacement of a surface element is equal to the driving force per unit area, i.e. the pressure $P$ resulting from all the conservative forces present, divided by the One-Body Dissipation coefficient $\rho \bar{v}$.

Applications:

a) The Wall Formula

The first illustration of Eq. (40) will be rather formal, since it will deal with shapes close to a sphere where the shell effects, neglected in this treatment, are expected to be especially strong. Nevertheless, this application brings out some interesting features of the theory.

Consider then an idealized nucleus whose shape deviations from the spherical configuration are described by the usual expansion in spherical harmonics

$$
R(\theta, \phi)=R\left[1+\sum_{\ell=1}^{\infty} \sum_{m=-\ell}^{\ell} a_{\ell m} Y_{\ell m}(\theta, \phi)\right] \text {. }
$$

The result of applying Eq. (40) to the motion of this slightly deformed 
surface can be shown to result in the following equation for the expansion coefficients $a_{\ell m}[8]$ :

$$
\dot{D} \dot{a}_{\ell m}+c_{\ell} a_{\ell m}=0,
$$

where

$$
D=R^{4} \rho \bar{v} \quad,
$$

and $c_{\ell}$ are the usual stiffness coefficients against distorting a charged drop by a multipole ripple of order $\ell[12]$ :

$$
C_{\ell}=\mathbf{R}^{2} \gamma\left[(\ell-1)(\ell+2)-\frac{20 \mathrm{X}(\ell-1)}{2 \ell+1}\right],
$$

Were $x$ ts the fissility parameter defined by $x=3 z^{2} e^{2} / 40 \pi R^{3} \gamma$.

If there had been no dissipation but instead the fluid flow had been assumed to follow irrotational hydrodynamics, the standard harmonic oscillator equation would have been found:

$$
M_{\ell} \ddot{a}_{\ell m}+C_{\ell} a_{\ell m}=0 \text {, }
$$

where $M_{\ell}=\rho R^{5} / \ell$ [12]. If both inertial and dissipative effects were present simultaneously, the equation of motion would be that of a damped harmonic oscillator:

$$
\mathrm{M}_{\ell} \ddot{\mathrm{a}}_{\ell \mathrm{m}}+\dot{\mathrm{D}}_{\ell \mathrm{m}}+\mathrm{C}_{\ell} \mathrm{a}_{\ell \mathrm{m}}=0 \text {. }
$$

The motion is overdamped or underdamped depending on the sign of the discriminant :

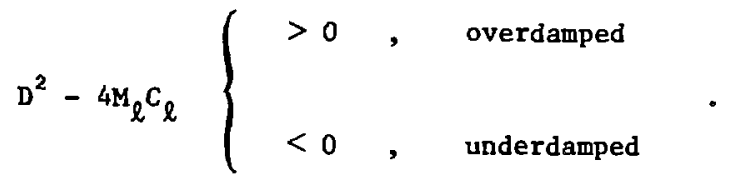

In Ref. [8] the "overdamping coefficients" $b_{/}^{\prime} 2 \sqrt{M_{\ell} C_{\ell}}$ are listed for a number of nuclei and multipole modes. For example, for $l=2$ and $20 \mathrm{Ne},{ }^{120} \mathrm{Sn}$, $238 \mathrm{~J}$, these coefficients are $1.51,2.62$, and 4.73 respectively. This would indicate moderate to strong overdamping (if shell effects could be disregarded).

The general motion of a strongly overdamped system can usually be divided intn two stages. Thus a steel ball dropped into a bucket of oil will first "thud", losing most of its kinetic energy during a short stage of violent (negative) acceleration. This is followed by a long "creep", with little kinetic energy and practically zero acceleration. In the thud stage the driving force is negligible, in the creep stage the inertial force Is negligible. In the case of Eq. (46) the thud stage would be governed by the equation

$$
M_{\ell} \ddot{a}_{\ell m}+D \dot{a}_{\ell m}=0
$$

and the creep stage by $\mathrm{Eq} .(42)$. The characteristic e-folding time for the thud is 


$$
\begin{aligned}
T_{\text {thud }}=\frac{M_{\ell}}{D} & =\frac{1}{\ell}(R / \bar{v}) \\
& =\frac{1}{2}(R / \bar{v}) \quad \text { for } \ell=2 .
\end{aligned}
$$

The e-folding time for the creep is

$$
\begin{aligned}
T_{\text {creep }} & =\frac{D}{C_{\ell}}=\left(R^{2} \rho \bar{v} / \gamma\right)\left[(\ell-1)(\ell+2)-\frac{20 \times(\ell-1)}{2 \ell+1}\right]^{-1} \\
& =\frac{\left(R^{2} \rho \bar{v} / \gamma\right)}{4(1-x)} \quad \text { for } \ell=2 .
\end{aligned}
$$

Equation (49) is a more quantitative statement of the order-oi-magnitude relation obtained in Lecture II, Eq. (31).

For a heavy nucleus $(R \approx 7 \mathrm{fm}, x \approx 0.75$ ) we find, approximately

$$
\begin{aligned}
& \mathrm{T}_{\text {thud }} \approx 0.5 \times 10^{-22} \mathrm{sec} \\
& \mathrm{T}_{\text {creep }} \approx 50 \times 10^{-22} \mathrm{sec}
\end{aligned}
$$

suggesting two fairly well separated time scales. (The overdamping coefficient is $\mathrm{D} / 2 \sqrt{\mathrm{M}_{\ell} \mathrm{C}_{\ell}}=\frac{1}{2} \sqrt{\mathrm{T}_{\text {creep }} / \mathrm{T}_{\text {thud }}} \approx 5$ in this case.) For lighter systems or higher multipoles the time scales become less distinct.

When a similar order-of-magnitude estimaie is made for nucleus-nucleus collisions with the aid of the window formula, there appears a geometrical factor which modifies the characteristic times for the thud and the creep. This factor is the ratio of the neck area $\pi a^{2}$, to a typical nuclear dinension $\pi R^{2}, i_{. e}(a / R)^{2}$. The thud time is lengthened by the factor $(R / a)^{2}$ and the creep time is shortened by the factor $(a / R)^{2}$, so that for small windows the distinction between the thud and the creep would be washed out.

These order-of-magnitude illustrations of the consequences of one-body damping were followed in Ref. [8] by an approximate solution of the equation of motion for a fissioning idealized nucleus in the presence of one-body damping, as described by the wall formula. Figure 15 compares the calculated kinetic energies of fission fragments at Infinity with measured values (for approximately symmetric division). The plot is for quclei from medium to heavy, as a function of the conventional parameter $z^{2} / A^{1 / 3}$. The dot-dashed curve is the result of a calculation for a non-viscous charged drop, and shows a tendency to overshoot the measured kinetic energies for heavy systems. The dashed curve shows how the kinetic energies can be lowered drastically by adding a larga ordinary viscosicy. (By adjusting the value of the viscosity coefficient one could, in fact, reproduce the measured values.) The solid curve shows the values predicted by the one-body dissipation theory in the approximation that uses the wall formula all the way to scission. The dynamics that results from this treatment is strongly damped (creepy) but, in contrast to the creepy division with a large ordinary viscosity, the predicted kinetic energies are not in violent disagreement with experiment. This is because the nature of the new dynamics (with one-body dissipation) is such that the scission shapes are relatively compact. The result is that there is enough electrostatic repulsion after sclssion to accelerate the fragments to about the correct final energy. This compactness of the fission shapes is illustrated in Fig. 16. Without viscosity the scission shapes are compact for light nuclei 
and moderately elongated for heavy nuclei. Large ordinary viscosity leads to more elongated shapes, especially for heavy systems. One-body dissipation, as approximated by the wall formula, leads to compact shapes for all systers.

Figure 17 shows the time evolution of the fissioning shapes (for ${ }^{236} U$ ) in a configuration space consisting of two dimensions, corresponding to distance between fragment centers (on the abscissa) and fragment elongation (on the ordinate).

These early comparisons suggested that there was no disaster when the onebody theory was confronted with experiment, even though the descent :rom saddle to scission was slow and creepy (which, if achieved by means of ordinazy viscosity, would have led to very much too low fragment kinetic energies). A serious reservation limiting the force of this comparison was the use of the wall formula also for necked-in shapes, where the formula is grossly in error. The result of switching to the more approprlate wall-plus-window formula will be discussed later.

\section{b) The Window Formula}

The window formula has been incorporated in several simplified treatments of nucleus-nucleus collistons. An early example is the study of the $197 \mathrm{Au}+86 \mathrm{Kr}$ reaction [8]. The two nuclei are approximated by rigid spheres interacting by the Proximity Potential and a generalization of the Window Formula that takes into account the diffuseness of the nuclear surfaces ("Proximity Friction"). There are no deformation or neck degrees of freedom, so it is obvious from the start that such a calculation is bound to be inadequate for the more intimate types of collisions, especially in the later stages of the process ("extt channel").

Figure 18 shows the time evolution of such an idealized $A u+K r$ colliston at Lab energies of 600,800 and $1000 \mathrm{MeV}$ and angular momentum $220 \mathrm{~h}$. (The case of $1000 \mathrm{MeV}$ and $280 \mathrm{~h}$ is also shown.) The relative CM energy is plotted vs. separation. The dots are spaced at intervals of $10^{-22} \mathrm{sec}$ and the latels are. current values of the angular momentum. The duration of the collision is of the order of 10 to $16 \times 10^{-22} \mathrm{sec}$ and a large fraction of the energy available above the interaction barrier is dissipated during the interaction. Figure 19 shows the scattering angle vs. final energy for the three Initial energies of 600, $800,1000 \mathrm{MeV}$ (Wilczyński plots). Figure 20 shows the final orbital angular momentum vs. the initial angular momentum. The value $l_{i \text { inal }}=(5 / 7) l_{\text {initial }}$ corresponds to the rolling condition which, under the restriction of the model, represents complete relaxation (i.e., the conversion of the maxtmum possible fraction of the relative $\pi$ tion into rotational degrees of freedom and heat). Frsm Fig. 20 one can see that a large fraction of the total reaction cross section would go into such completely relaxed collisions. $[13,14]$.

More extensive calculations along these lines have been carried out in Refs.

Because of the neglect of deformation and neck degree: of freedom one should not attempt a direct comparison of such calculations with experiment. What the calculations do illustrate is that the one-body dissipacion, as described by the window formula, is a powerful mechanism for dissipating the energy of relative motion in nucleus-nucleus collisions.

\section{c) The Wal1-Plus-W1ndow Formula: Fission}

Recently [15] the fission calculations which I described earlier have been generalized to correct the unrealistic use of the wall formula near scission. In the new calculations the wall formula is used until the neck radius has reached a prescribed value, after which a switch is made to the wall-plus-window formuia, appropriate to small necks. (In the calculations of Ref. [15] a simplified verston 
of the wall-plus-window formula was used, in which the wall formula was applied for each fragment using the center-of-mass motion to represent the fragments' drifts $D_{1}, D_{2}$ in Eq. (23), Lecture II.) The effect of this procedure on the fission-fragment kinetic energies is shown in Fig. 21. The curve labeled "transition neck radius $=0$ " corresponds to using the wall formula all the way to scission, as in Fig. 15. (The slight difference between the two curves is due to a slightly different method used in calculating the potential energy.) The curve labeled "cylindrical neck" corresponds to making the transition to the wall-pluswindow formula when the neck is about to appear (i.e., when the conic used to represent the neck region is a portion of a cylinder). An optimal calculation would presumably correspond to making the transition between these two extremes. The curves labeled $2.5 \mathrm{fm}$ and $3.0 \mathrm{fm}$ make the transition when the radius of the neck has the value indicated. Figure 22 shows how, in the case of ${ }^{236} \mathrm{U}$, the kinetic energy varies with Increasing transition neck radius. After an initial inzrease of a couple of $\mathrm{MeV}$ the kinetic energy decreases and levels out some 13 $\mathrm{MeV}$ below the value of $170 \mathrm{MeV}$, corresponding to making the transition at scission. This decrease was expected from the qualitative argument that switching to the wall-plus-window form was we ellew us making the dynaws wure like the dynamics with ordinary viscos'ty, leading to scmewhat less compact shapes. This is 1llustrated in Fig. 23 (similar to Fig. 17).

The result of these studies is that for a switch from the wall to the wallplus-window formula at a point intermediate between the two extremes considered, the one-body dissipation theory suggests: (a) scission shapes somewhat more compact than in the reference calculation without viscosity, (b) scission energies of some 10 to $15 \mathrm{MeV}$ for heavy nuclei, (c) final fragment kinetic energies that are not in violent disagreement with measured values throughout the periodic table.

\section{d) The Wall-Plus-Window Formula: Nucleus-Nucleus Coll1sions}

The last application I would like to describe is a simplified treatment of the dynamics of nucleus-nucleus collisions using the wall-plus-window formula, which should be valid when the neck between the two nuclei is not too large. To make the problem even semi-analytic some drastic approximations are necessary, in addition to the idealization that goes into the one-bndy dissipation theory. Thus we parameterize the shapes of fusing or re-separating nuclei, as described by the half-density contour, by two spheres connected by a cylindrical neck (see Fig. 24). The radil of the spheres will be written as $R_{1}, R_{2}$ or $C_{1}, C_{2}$. (We shall disregard in what follows the difference, of order $b^{2}$, betwaen the effective sharp radius $R$ and the half-density radius $C$.) The surfaces of the spheres are separated by $s$ (so their centers are $R_{1}+R_{2}+s \approx C_{1}+C_{2}+s \equiv z$ apart). The neck radius is $n$. In: what foilows we shall assume that the neck, in particular its volume, is small. For a small neck its length $\ell$ may be shown to ke given by

$$
\ell \approx \varepsilon+n^{2} / 2 \bar{R},
$$

where $\bar{R}=C_{1} C_{2} /\left(C_{1}+C_{2}\right) \approx R_{1} R_{2} /\left(R_{1}+R_{2}\right)$.

Introducing the untt of length $u_{\ell} \equiv 2 \overline{\mathrm{R}}$ and using the definitions

$$
\begin{aligned}
& \sigma \equiv \mathbf{s} / 2 \overline{\mathrm{R}} \\
& \nu \equiv \mathrm{n} / 2 \overline{\mathrm{R}} \\
& \lambda \equiv \ell / 2 \overline{\mathrm{R}}
\end{aligned}
$$

te may re-write Eq. (51) as

$$
\lambda \approx \sigma+v^{2}
$$


For a given abyumetry $\left(R_{1}: R_{2}\right)$ we shall take $\sigma, v$ as the degrees of freedom describing the system. Since he neck radius and neck length cannot be negative, the configuration space $\sigma, v$ is limited by the boundaries corresponding to

$$
\begin{aligned}
& v>0, \\
& \sigma>-v^{2},
\end{aligned}
$$

In the case when the nuclel have diffuse surfaces, Eq. (53) has to be modified because the half-density contour of a system consisting of two diffuse spheres develops a neck before the half-density radi1 $c_{1}, c_{2}$ have touched. Taking the case of a trapezoidal density profile as an example (1.e., a linear fall-off of the density from its bulk value to zero in a total distance $B$, say), a neck in the half-density contour of the total system w111 form when the separate quarterdensity points have touched at $s=s_{1}=\frac{1}{2} B$. It may be verified that, as the surfaces apprcach fur ther, the half-density contour develops a neck which is, at first, exactly cylindrical (In the case of the linear diffuseness that we are constdering). The radius of this "geometrical" neck is given by

$$
\mathrm{n}_{\mathrm{g}}=\sqrt{2 \overline{\mathrm{R}}\left(\mathrm{s}_{1}-\mathrm{s}\right)}+\text { higher orders of } s / 2 \overline{\mathrm{R}} \text {. }
$$

Since, for a linear surface profile, the Süssmann width $b$ is related to the total width $B$ by $B=2 \sqrt{3} b$, the critical distance at which the geometrical neck first appears is given by $s_{1}=\sqrt{3} b \approx 1.73 \mathrm{fm}$. It follows from Eq. (54) that the effect of surface diffuseness is to move the boundary of the configuration space from $\sigma=-v^{2}$ [Eq. (53)] to

where

$$
\sigma=\sigma_{1}-v^{2},
$$

$$
\sigma_{1}=s_{1} / 2 \bar{R} \text {. }
$$

The configuration space of the degrees of freedom $s, n$ is shown in Fig. 25 . When plotted with $2 \bar{R}$ as a unit of length the resulting configuration space of $\sigma, v$ is independent of asymetry. The three-dimensional configuration space of $s, n$ and $R_{1}: R_{2}$ could be constructed by imagining appropriately scaled sections like the upper part of Fig. 25 stacked one behind the other. In the dynamical situations that we shall consider, for which the neck between the two fragments is assumed to be not too large, the flow of matter between the fragments will be 1mpeded by the neck constriction and the asymmetry degree of freedom w111 be characterized by a relatively longer time scale. There will then be some measure of validity in the assumption that the asymetry $R_{1}: R_{2}$ remains approximately frozen and can be treated as a fixed parameter rather than a dynamical variable. There remain, therefore, in the present approximation, two dynamical degrees of freedom, $\sigma$ and $v$.

The potentiai energy associated with a shape specified by $\sigma, \nu$ is estimated as

$$
\mathrm{PE}=\mathrm{E}_{\text {coulomb }}+\mathrm{E}_{\text {nuclear }} \text {, }
$$

where we shall take

$$
\begin{aligned}
E_{\text {coulomb }} & \approx \frac{3}{5} \frac{\left(z_{1} e\right)^{2}}{R_{1}}+\frac{3}{5} \frac{\left(z_{2} e\right)^{2}}{R_{2}}+\frac{z_{1} z_{2} e^{2}}{R_{1}+R_{2}+s}, \quad \text { for } s>0 \\
& \approx \frac{3}{5} \frac{\left(z_{1} e\right)^{2}}{R_{1}}+\frac{3}{5} \frac{\left(z_{2} e\right)^{2}}{R_{2}}+\frac{z_{1} z_{2} e^{2}}{R_{1}+R_{2}}-\frac{z_{1} z_{2} e^{2}}{\left(R_{1}+R_{2}\right)^{2}}, \text { for } s<0 .
\end{aligned}
$$


and

where

$$
E_{\text {nuclear }} \approx 4 \pi Y\left(R_{1}^{2}+R_{2}^{2}\right)+2 \pi Y\left[n\left(\ell-\ell_{f}\right)-n^{2}\right],
$$

$$
l_{f}=s_{1}\left(1-\frac{n}{2 \bar{R}}\right)
$$

Equation (56) is the surface energy (in the small-neck approximation) of the dumb-bell in Fig. 24, with a proximity correction that assigns zero surface energy to a certain length $l_{f}$ of the cylindrical part of the neck. This is in the spirit of the discussion following Eq. (15e), according to which surface elements within an effective interaction range $s_{1}$ of the spherical surfaces should be assigned zero surface energy. This effective interaction range $s_{1}$ is actually modified somewhat in Eq. (57) by the factor $(1-n / 2 \vec{R})$. This factor makes the proximity correction decrease as the neck fills in, so that by the time the indentation in the nuclear configuration has disappeared, the Proximity correction is gone. (For equal spineres with radil $R_{1}$ this occurs at $n=2 \bar{R}=R_{1}$. For very unequal spheres, of which the smaller has radius $R_{1}$, we have $\bar{R} \approx R_{1}$, so that when $n=R_{1}$ the factor $1-n / 2 \bar{R}$ is equal to $\frac{1}{2}$. This is reasonable since in this case only one of the two indentations in the shape has disappeared.)

Note that the total potential energy, taken with respect to the energy $\mathrm{PE}_{\infty}$ of infinitely separated fragments, and written in units of $8 \pi \gamma \bar{R}^{2}$, may be put in the following compact form:

$$
\begin{aligned}
E(\sigma, v) \equiv\left(P E-P E_{\infty}\right) / 8 \pi \gamma \overline{\mathrm{K}}^{2}= & v\left(\sigma-\sigma_{1}\right)-v^{2}\left(1-\sigma_{1}\right)+v^{3} \\
& +X \begin{cases}\frac{1}{\Lambda+\sigma} & \text { for } \sigma>0 \\
\frac{1}{\Lambda}-\frac{\sigma}{\Lambda^{2}} & \text { for } \sigma<0,\end{cases}
\end{aligned}
$$

where $X$ stands for the "Coulomb Parameter" $z_{1} z_{2} \mathrm{e}^{2} / i 6 \pi \gamma \overline{\mathrm{R}}^{3}$.

A typical potential-energy landscape represented by Eq. (57a) is shown in Fig. 26. There are two regions of low energy, separated by a saddle point pass. Explicit expressions $\sigma(v)$ for the equipotential lines are obtained by setting $\varepsilon$ equal to a constant and solving $\mathrm{Eq}$. (57a) for $\sigma$ :

$$
\begin{array}{ll}
\sigma=\left[\frac{x}{\Lambda}-\varepsilon-v \sigma_{1}-v^{2}\left(1-\sigma_{1}\right)+v^{3}\right] /\left(\frac{x}{\Lambda^{2}}-v\right) & \text { for } \sigma<0 \\
\sigma=\frac{1}{2}\left[-B \pm \sqrt{B^{2}-4 C}\right], & \text { for } \sigma>0
\end{array}
$$

where

$$
\begin{aligned}
& B=\Lambda-\frac{\varepsilon}{v}-\sigma_{2}-\left(1-\sigma_{1}\right) v+v^{2} \\
& C=\frac{x}{v}+\Lambda(B-\Lambda) .
\end{aligned}
$$

The location of the saddle point is found by solving the pair of equations

$$
\frac{\partial \varepsilon}{\partial \sigma}=0, \quad \frac{\partial \varepsilon}{\partial \nu}=0,
$$

which make the energy stationary. Since, in general, the energy is not stationary 
wth respect to the asymetry degree of freedom, these are "conditional" saddle points with the physical meaning of a mountain pass only if the asymmetry is effectively held fixed. For a reflection symetric system the energy is stationary with respect to asymmetry and the conditlonal saddle becomes a true, unconditional saddle. This saddle separates the configuration space into two regions of low potential energy, one corresponding to a compound nucleus and the other corresponding to a disintegrated configuration of fragments at infinity. It is also well known that for not too light systems the energy may be made stationary with respect to asymmetry if the asymmetry is chosen large enough [16]. These "Businaro-Gallone" shapes of unstable equilibrium divide the range of asymetric conditional saddle configurations into two classes: those (with asymetrles less than a critical value) that are driven towards symetry, and those (with asymmetries greater than a critical value) that are driven towards even greater asymetries. Near the critical value itself there is little driving force to change the asymetry. Some properties of the unconditional saddle point configurations that result from setting the diffuseness parameter $\sigma_{1}$ to zero in Eq. (57a) (and taking $\Lambda=2$, corresponding to symmetry), are illustrated in Figs. 27 and 28 . Figure 27 shows the neck radius $n$ and the separation between fragment centers $z$, both in units of $2 \bar{R}$, as a function of the fissility parameter $x$ [related, for symetric systems, to our Coulomb parameter $X$ by $x=(3 / 10) X]$. Figure 28 shows the energies of these saddle points plotted conventionally with respect to the energy of a single sphere of equal volume and in units of the surface energy $4 \pi R^{2} \gamma$ of that sphere. This enables one to compare the saddle point energies calculated with our potentlal energy expression (based on a restricted dumb-bell parameterization and on using a small-neck approximation) with exact results for unrestricted sharp-surface drops. We note from Figs. 27 and 28 that the approximate calculations reproduce very roughly the familiar rapid transition from necked-in to cylinder-like saddle shapes and that the trend of saddle energies is also roughly correct even for $x$-values approaching unity and saddle shapes approaching a sphere, where our approximations are not meant tu hold and should not be trusted. In practical applications the correspondence between the approximate and exact results could be further enhanced by a slight re-scaling of the fissility parameter $x$ (for example, by the choice of a surface energy coeffictent $\gamma$ reduced by some $15 \%$ ).

So much for the Potential Energy.

As an approximation to the Kinetic Energy of the configuration in Fig. 24, when this configuration is in a state of deformation, we shall use

$$
\mathrm{KE} \approx l_{2} \mathrm{M}_{\mathrm{r}} \dot{\mathrm{s}}^{2} \quad \text {, }
$$

where $M_{r}$ is the reduced mass of the system:

$$
\begin{aligned}
M_{r} & =M_{1} M_{2} /\left(M_{1}+M_{2}\right) \\
& =m A_{1} A_{2} /\left(A_{1}+A_{2}\right) .
\end{aligned}
$$

Here m is a nuclear mass ur.it $\left(\mathrm{mc}^{2} \approx 931 \mathrm{MeV}\right.$ ) and $A_{1}, A_{2}$ are the mass numbers of the two fragments. In Eq. (58) there is no term in $\vec{n}$, which corresponds to the assumption that the motion in the neck degree of freedom is strongly overdamped.

For the Rayleigh Dissipation Function, DF, we shall use the Wa1l-plus-Window formula, which leads to

$$
\begin{aligned}
\mathrm{DF} & \equiv \frac{2}{2} \dot{Q} \\
& =\frac{1}{4} \pi \bar{v}^{2} \dot{\mathrm{s}}^{2}+\pi \rho \bar{v} \ln \dot{\mathrm{n}}^{2}
\end{aligned}
$$


The equations of motion follow from the canonical equations

$$
\begin{aligned}
& \frac{d}{d t} \frac{\partial(K E)}{\partial s}+\frac{\partial(D F)}{\partial \dot{s}}+\frac{\partial(P E)}{\partial s}=0 \\
& \frac{d}{d t} \frac{\partial(K E)}{\partial \dot{n}}+\frac{\partial(D F)}{\partial \dot{n}}+\frac{\partial(P E)}{\partial n}=0 .
\end{aligned}
$$

It may be verified that if we adopt the time unit $u_{t}$ defined by

$$
u_{t}=\rho \bar{v} \bar{R}^{2} / \gamma
$$

and write $t / u_{t} \equiv \tau$, the equations of motion reduce to the relatively clean expressions

$$
\begin{aligned}
& \mu \ddot{\sigma}+\nu^{2} \dot{\sigma}= \begin{cases}\frac{x}{(\Lambda+\sigma)^{2}} & \text { for } \sigma>0 \\
\frac{x}{\Lambda^{2}}-\nu & \text { for } \sigma<0\end{cases} \\
& 4 \nu\left(\sigma+\nu^{2}\right) \dot{\nu}=\left(\sigma_{1}-\sigma\right)+2\left(1-\sigma_{1}\right) \nu-3 \nu^{2} .
\end{aligned}
$$

In these equations there are four dimensionless parameters: $\chi, \mu, \sigma_{1}$, and $\Lambda$ given by

$$
\begin{aligned}
X & =\text { Coulomb parameter } \\
& \equiv \mathrm{Z}_{1} \mathrm{Z}_{2} \mathrm{e}^{2} / 16 \pi \gamma \overline{\mathrm{R}}^{3} \\
\mu & =\text { Inertia parameter } \\
& \equiv \mathrm{M}_{\mathrm{r}} \gamma / 2 \pi(\rho \overline{\mathrm{v}})^{2} \overline{\mathrm{R}}^{4} \\
\sigma_{1} & =\text { Diffuseness parameter } \\
& \equiv \sqrt{3} \mathrm{~b} / 2 \overline{\mathrm{R}} \\
\Lambda & =\text { Asymetry parameter } \\
& \equiv\left(\mathrm{R}_{1}+\mathrm{R}_{2}\right) / 2 \overline{\mathrm{R}}
\end{aligned}
$$

The values of these parameters are 1isted in Table II for symmetric systems $\left(A_{1}=A_{2}=\frac{1}{2} A\right)$ in the range $A=20$ to $A=300$ and for $Z$ va ises along a nominal valley of beta-stability, given by $Z=\frac{1}{2} A(I-I)$, where $I=(N-Z) / A$ and

$$
I=\frac{0.4 \mathrm{~A}}{\mathrm{~A}+200} \text {. }
$$

[We have used $\gamma=0.9517\left(1-1.7826 \mathrm{I}^{2}\right), I_{0}=1.18 \mathrm{fm}$. These are rough estimates, convenient for illustrative purposes.] 
TABLE II. Saddle-point properties for nuclei with mass numbers $A=20-300$ and atomic numbers $Z=\frac{1}{2} A(1-I)$, where $I=0.4 \mathrm{~A} /(200+\mathrm{A})$. The saddles are reflection symmetric $(\Lambda=2)$. The fissility parameter $x$ is $(3 / 10)$ times the Coulomb parameter $X$ given by $E q .(66)$, the diffuseness parameter $\sigma_{1}$ is given by Eq. (68) and the Inertia parameter $\mu$ by Eq. (67). The saddle-point locations $\nu_{s p}, \sigma_{s p}$ were obtained by solving Eqs. (57b).

\begin{tabular}{|c|c|c|c|c|c|c|}
\hline A & 2 & $x$ & $\sigma_{1}$ & $\mu$ & $v_{\text {sp }}$ & $\sigma_{\text {sp }}$ \\
\hline 20 & 9.636 & 0.10230 & 0.68131 & 0.34970 & 0.04662 & 0.70450 \\
\hline 40 & 18.667 & 0.19302 & 0.54076 & 0.27601 & 0.09511 & 0.60098 \\
\hline 60 & 27.231 & 0.27586 & 0.47239 & 0.23935 & 0.14016 & 0.56136 \\
\hline 80 & 35.429 & 0.35311 & 0.42920 & 0.21568 & 0.18278 & 0.53764 \\
\hline 100 & 43.333 & 0.42628 & 0.39843 & 0.19849 & 0.22422 & 0.51737 \\
\hline 120 & 51 & 0.49637 & 0.37494 & 0.18517 & 0.26573 & 0.49530 \\
\hline 140 & 58.470 & 0.56408 & 0.35616 & 0.17438 & 0.30876 & 0.46775 \\
\hline 160 & 65.778 & 0.62993 & 0.34066 & 0.16539 & 0.35551 & 0.43030 \\
\hline 180 & 72.947 & 0.69428 & 0.32754 & 0.15773 & 0.41066 & 0.37392 \\
\hline 194 & 77.895 & 0.73860 & 0.31946 & 0.25300 & & \\
\hline 196 & 78.598 & 0.74489 & 0.31837 & 0.15236 & & \\
\hline 198 & 79.300 & 0.75116 & 0.31730 & 0.15172 & 0.48290 & 0.27708 \\
\hline 200 & 80 & 0.75743 & 0.31624 & 0.15110 & 0.49553 & 0.25723 \\
\hline 202 & 80.700 & 0.76369 & 0.31519 & 0.15048 & 0.51155 & 0.23077 \\
\hline 204 & 81.398 & 0.76994 & 0.31416 & 0.14988 & 0.54003 & 0.18000 \\
\hline 206 & 82.096 & 0.77618 & 0.31314 & 0.14928 & 0.64682 & -0.05344 \\
\hline 208 & 82.792 & 0.78240 & 0.31213 & 0.14868 & 0.65200 & -0.06620 \\
\hline 220 & 86.952 & 0.81959 & 0.30635 & 0.14528 & c 68299 & -0.09966 \\
\hline 240 & 93.818 & 0.88093 & 0.29759 & 0.14012 & 0.73411 & -0.17271 \\
\hline 260 & 100.609 & 0.94158 & 0.28975 & 0.13549 & 0.78465 & -0.26580 \\
\hline 280 & 107.333 & 1.00165 & 0.28268 & 0.13132 & 0.83471 & -0.37764 \\
\hline 300 & 114 & 1.06122 & 0.27629 & 0.12754 & & \\
\hline
\end{tabular}

One notes that, compared to $X$, the parameters $\mu$ and $\sigma_{1}$ vary relatively slowly in the above range of A-values. This is also true of the parameter $\Lambda$, which is approximately equal to 2 except for very asymmetric fragments. For a range of medium-weight nuclear systems one might, therefore, simplify Eqs. $(64,65)$ still further by putting $\mu \approx 1 / 6, \sigma_{1} \approx 1 / 3, \Lambda \approx 2$. This gives the "Pedagogical Equations":

$$
\frac{1}{6} \ddot{\sigma}+v^{2} \dot{\sigma} \approx\left\{\begin{array}{ll}
\frac{x}{(\Lambda+\sigma)^{2}}-\nu & \text { for } \sigma>0 \\
3 x-\nu & \text { for } \sigma<0
\end{array},\right.
$$




$$
4 v\left(\sigma+v^{2}\right) \dot{v} \approx\left(\frac{1}{3}-\sigma\right)+\frac{4}{3} v-3 v^{2}
$$

These equations stress the fact that there is one dominant dimensionless parameter $X$ in the dynamical theory as formulated above. For symmetric systems this parameter reduces in fact to $10 / 3$ times the conventional fissility parameter $x$. We may thus define a generalized fissility or Coulomb parameter $\mathrm{X}$ by

$$
\begin{aligned}
\mathrm{X} \equiv \frac{3}{10} x & =3 z_{1} z_{2} \mathrm{e}^{2} / 1 \in 0 \pi \gamma \overline{\mathrm{R}}^{3} . \\
& =\left(\frac{\mathrm{Z}^{2}}{\mathrm{~A}}\right)_{\text {eff }} /\left(\frac{\mathrm{Z}^{2}}{\mathrm{~A}}\right)_{\text {crit }},
\end{aligned}
$$

where $\left(\mathrm{Z}^{2} / \mathrm{A}\right)_{\text {crit }} \equiv 40 \pi \gamma \mathrm{r}_{0}^{3} / 3 \mathrm{e}^{2} \approx 50$, as in the case of fission, and

$$
\left(\frac{Z^{2}}{A}\right)_{\text {eff }} \equiv \frac{Z_{1} Z_{2}\left(A_{1}^{1 / 3}+A_{2}^{1 / 3}\right)^{3}}{4 A_{1} A_{2}}
$$

A consequence of the dominance of the problem by the single parameter $X$ is the prediction that the dynamics of two (possibly) quite different di-nuclear systems should be similar (in the sense of being approximately related to each other by a scaling with the appropriate length and time units $u_{\ell}=2 \bar{R}$ and $u_{t}=\rho \bar{v} \bar{R}^{2} / \gamma$ ) provided the values of $X$ (i.e, of $\left(Z^{2} / A\right)$ eff) are chosen to be equal for the two systems. It also follows that, as in fission, it should be useful to plot certain experimental results on collisions between different nuclear systems as a function of the generalized fissility parameter $X$ or the coulomb index $\left(Z^{2} / A\right)_{\text {eff }}$.

In the case when an angular momentum $L$ is present, the centrifugal forces increase the disruptive tendency of the Coulomb repulsion. A very rough way to take this into account would be to augment the Coulomb energy in Eq. (55) by a centrifugal energy

$$
\mathrm{E}_{\text {centr }}=\frac{\mathrm{L}^{2}}{2 \mathscr{J}} \text {, }
$$

where $\mathscr{V}$ is an appropriate moment of inertia. In the inftial stages of the collision $\mathcal{J}$ would be close to the "mass-point" value

$$
\mathscr{J} \approx \mathrm{M}_{\mathrm{r}} z^{2}
$$

If the collision is sufficiently intimate the "rigid-body" value might be more relevant in the later stages (but not after re-disintegration):

$$
\mathcal{J} \approx M_{r} z^{2}+\frac{2}{5} M_{1} R_{1}^{2}+\frac{2}{5} M_{2} R_{2}^{2}
$$

The centrifugal force to be added to Eq. (61) would be

$$
-\frac{\partial}{\partial z} \frac{L^{2}}{2 J}=L^{2} \frac{M_{r} z}{J^{2}}
$$

One might try to mock up the presence of the centrifugal force by an increase in the electric repulsion. By matching the centrifugal force at contact $\left(z=R_{1}+R_{2}\right)$ by an electric force $z_{1} z_{2} e^{2} /\left(R_{1}+R_{2}\right)^{2}$, this mocking up would mean that the Coulomb parameter $X$ in $\mathrm{Eq}$. (64) would be multiplied by the factor 


$$
1+\frac{L^{2} M_{r}\left(R_{1}+R_{2}\right)}{\mathscr{J}_{0}^{2}} / \frac{z_{1} z_{2} e^{2}}{\left(R_{1}+R_{2}\right)^{2}},
$$

where $\mathscr{V}_{0}$ stands for Eqs. (77) or (78) evaluated at $z=R_{1}+R_{2}$. Thus in the equations of motion 64,65 (or 71,72 ) the parameter $X$ now stands for a

$$
\text { Disruption Parameter } \chi=\chi_{\text {Coulomb }}+\chi_{\text {centrifugal }} \text {, }
$$

where

$$
\begin{aligned}
& X_{\text {Coulomb }}=z_{1} z_{2} e^{2} / 16 \pi \gamma \bar{R}^{3}, \\
& X_{\text {centrifugal }}=L^{2} M_{r}\left(R_{1}+R_{2}\right)^{3} / 16 \pi \gamma \bar{R}^{3} \mathscr{O}_{0}^{2} .
\end{aligned}
$$

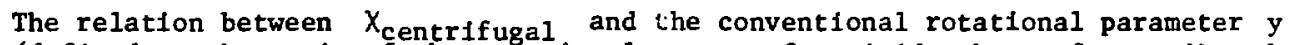
(defined as the ratio of the rotational energy of a rigid sphere of mass $M$ and radius $R$ to its surface energy, see Ref. [17]) is analogous to the relation between $X_{\text {Coulomb }}$ and the fissility parameter $x$. Thus if we define a generalized rotational parameter $\mathrm{Y}$ by

$$
Y \equiv\left(\frac{49}{160.2^{1 / 3}}\right) X_{\text {centrifugal }}
$$

we find that for a symmetric system $\left(M_{1}=M_{2}=\frac{1}{2} M, R_{1}=R_{2}=R / 2^{1 / 3}\right)$ and with the rigid-body value for $\mathscr{J}_{0}$ :

$$
\begin{aligned}
\mathcal{V}_{0}^{\mathrm{rlg} I \mathrm{~d}} & =M_{r}\left(\mathrm{R}_{1}+\mathrm{R}_{2}\right)^{2}+\frac{2}{5} M_{1} \mathrm{R}_{1}^{2}+\frac{2}{5} \mathrm{M}_{2} \mathrm{R}_{2}^{2} \\
& =\frac{7}{5} \mathrm{MR}^{2} / 2^{2 / 3},
\end{aligned}
$$

the expression for $Y$ reduces to

$$
Y \rightarrow \frac{5}{16 \pi} \frac{L^{2}}{M R^{4} \gamma}=\frac{L^{2}}{2\left(\frac{2}{5} M^{2}\right)} / 4 \pi R^{2} \gamma,
$$

which is the definition of $y$. The disruption parameter $X$ in Eqs. $(71,72)$ can now be written as

$$
\begin{aligned}
X & =\frac{10}{3} X+\frac{160.2^{1 / 3}}{49} Y \\
& =\frac{10}{3}\left(X+\frac{48}{49} 2^{1 / 3} Y\right)
\end{aligned}
$$

We thus arrive at a rough generalized scaling rule: "For two di-nuclear systems with possibly quite different sizes, asymetrles and angular momenta, the dynamical time evolutions in the configuration space of neck and separation can be approximately scaled Into each other provided the disruption parameters $\chi$ for the two systems are about equal." Equation (86), In particular, tells how a certain amount of electric repulaion can be traded of $f$ for an approximately equivalent amount of centrifugal repulsion. Thus Eq. (86) says that systems specifled by 


$$
\mathrm{X}+(48 / 49) 2^{1 / 3} \mathrm{Y}=\text { constant }
$$

should be similar. In a parameter space of $Y$ vs. $X$ these are lines with a slope

$$
\frac{d Y}{d X}=-\frac{49}{48.2^{1 / 3}}=-0.8102 \approx-4 / 5 \text {, }
$$

so that a given amount of angular momentum specified by $Y$ is, for di-nuclear systems, about five-fourths more efficient in its disruptive effects thin an electrostatic energy spectfled by the same value of $x$. These scaling ruies are only applicable (roughly) for di-nuclear systems with not too large necks. They should not be applied to situations where shapes relevant to the problem at hand are not necked-ir (and the rigid-body moment of inertia must not be used before contact or after re-disintegration).

For grazing collisions, where the "mass-point" value of $\mathcal{J}_{0}$ would be more appropriate, the generalized rotational parameter Y, given by Eq. (83), would have (for the same system and angular momentum) a numerical value greater by the factor $\left(\mathcal{J}_{0}^{\mathrm{rig} i d} / \mathcal{J}_{0} \text { points }\right)^{2}$. This factor is equal to $49 / 25$ for a symetric system and becomes much larger for asymetric systems. Thus a given amount of angular momentum is a factor of at least two more efficient in its disruptive tendency in grazing collisions (or in the early stages of any collision) than in more intimate collisions, when a rigid-body type of rotation may have established itself.

With the equations of motion $(64,65)$ or $(71,72)$ written down, we are now ready to look at some dynamical trajectories in $\sigma-v$ space representing nucleus-nucleus collisions (see Fig. 29). The approa-h of two nuclei is represented by a point moving from right to left along the $\sigma$-axis. At the point $\sigma=\sigma_{1}$ the neck begins to grow along the geometrical locus

$$
v=\sqrt{\sigma_{1}-\sigma}
$$

merely by virtue of the superposition of the diffuse density distributions of the two nuclei. For a given speed of approach $\dot{\sigma}$ the rate of neck growth $\dot{v}$ is

$$
v=\frac{-\dot{\sigma}}{2 \sqrt{\sigma_{1}-\sigma}}=\frac{-\dot{\sigma}}{2 \nu},
$$

which tends to infinity for small values of $\nu$, when $\sigma$ is close to $\sigma_{1}$. It follows that the neck-growth speed, as given by the geometrical Eq. (90), is certain to exceed initially the speed given by the dynamical Eq. (65). The initial stage of the collision would proceed, therefore, along the geometrical locus, with $v$ related to $\sigma$ by Eq. (89). Consequently the dynamical problem continues for a while to be one-dimensional even after contact at $\sigma=\sigma_{1}$. The potential energy for this problem is obtained by substituting Eq. (89) into Eq. (57a):

$$
\begin{aligned}
& E(\sigma)=\left(1-\sigma_{1}\right)\left(\sigma-\sigma_{1}\right) \\
& +x \begin{cases}\frac{1}{\Lambda+\sigma} & \text { for } \sigma>0 . \\
\frac{1}{\Lambda}-\frac{\sigma}{\Lambda^{2}} & \text { for } \sigma<0 .\end{cases}
\end{aligned}
$$


The negative derlvative with respect to $\sigma$ gives the driving force, and balancing this against the inertial and disslpative forces leads to the following single equation of motion for $\sigma$ (1n place of the coupled equations $(64,65)$ :

$$
\mu \ddot{\sigma}+\left(\sigma_{1}-\sigma\right) \dot{\sigma}=\sigma_{1}-1+ \begin{cases}\frac{x}{(\Lambda+\sigma)^{2}} & \text { for } \sigma>0, \\ \frac{x}{\Lambda^{2}} & \text { for } \sigma<0 .\end{cases}
$$

Equation (92a) can be solved analytically if the right-hand side is approximated by a constant (as 15 already the case for Eq. (92b)). For example, putting $\sigma=\sigma_{1}$ on the right of Eq. (92a), we find

$$
\ddot{\sigma}+\frac{\sigma_{1}-\sigma}{\mu} \dot{\sigma}=-G
$$

where $G$, a constant, stands for $\left(i-\sigma_{1}-\frac{X}{\left(\Lambda+\sigma_{1}\right)^{2}}\right) / \mu$.

The solution of Eq. (93), whIch at $T=0$ starts off with $\sigma=\sigma_{1}$ and $\dot{\sigma}=\dot{\sigma}_{0}$, Is found to be given by

$$
\sigma-\sigma_{1}=-\left(4 \mu^{2} G\right)^{1 / 3} \frac{F^{\prime}(z)}{F(z)}
$$

where

$$
\begin{aligned}
& z=z_{1}+\left(\frac{G}{2 \mu}\right)^{1 / 3} \tau, \\
& z_{1}=-\frac{\dot{o}_{O}}{G}\left(\frac{G}{2 \mu}\right)^{1 / 3}
\end{aligned}
$$

and $F(z)$ satisfies the differential equation

$$
F^{\prime \prime}=2 F \quad \text {. }
$$

Th1s means that $F(z)$ is a superposition of the Airy functions $A(2)$ and $B 1(z)$ [18]

$$
F(z)=A i(z)+k B i(z) \text {. }
$$

It may be verified that, In order to satisfy the initial condition, the constant $k$ must have the value

$$
k=-\frac{A 1^{\prime}\left(z_{1}\right)}{B 1^{\prime}\left(z_{1}\right)}
$$

The above geometrical, one-dimensional evolution continues until the velocity given by Eq. (65) has attained equality with the geometrlcal velocity given by Eq. (90), at which stage the trajectory in $\sigma-V$ space peels off tangentially from the geometrical locus. The condition for peeling off is obtained by equating Eqs. (65) and (90). Using Eq. (89) this condition may be written as the following simple relation between the velocity $\dot{\sigma}$ and location $\sigma, v$ at the moment of peeling off: 


$$
\begin{aligned}
& \dot{\sigma}=-v\left(1-\sigma_{1}-v\right) / \sigma_{i}, \\
& \sigma=\sigma_{1}-v^{2},
\end{aligned}
$$

Equations (100) and (101), which do not require the solution of the geometrical one-dimensional stage, may be used to specify the injection conditions into the two-dimensional dynamical equations of motion, Eqs. $(64,65)$.

Figure 29 shows seven dynamical trajectories for a system with a total mass number $A=160$ and $Z=65.778$ (see Table $I I$ ). This could represent the head-on collision of two equal nuclei with $A_{1}=A_{2}=80, z_{1}=z_{2}=32.89$ or, approximately, the collisions of unequal nuclei (perhaps with angular momentum) for which the disruption parameter $X$ has the same value [about $(10 / 3)(0.63) \approx 2.1$ ]. The trajectories are labeled with the value of $v$ for which peeling oft from the geometrical locus has taken place. The trajectory labeled $v=0$ corresponds to starting off at the top of the interaction barrier. A rapid growth of the neck radius is followed by a neck elongation and a final abrupt collapse of the neck. The trajectorles with $v=0.10, v=0.11$ are similar except that the time taken to re-separate becomes progressively longer as the trajectories come into the neighborhood of the saddle-point configuration, where the potential energy is stationary. (The dots along the trajectories correspond to time intervals of one-tenth of the natural time unit $\left.u_{t}=\rho v R^{2} / \gamma_{.}\right)$The trajectory labeled $v=0.13$ actually passes on the inside of the saddle point but nevertheless leads to re-separation. The trajectories with $v=0.14,0.15,0.20$ lead to capture on the inside of the saddle point. Figure 26 illustrates the topography of the potential energy surface on which the above dynamical evolutions are taking place. The peeling off configurations with $v=0,0.1, \ldots 0.2$ correspond to points located on the southwest ridge overlooking the saddle point. The higher values of $v$ correspond to points more nearly "inside" the saddle configuration and this, combined with the greater injection velocity $-\dot{\sigma}$ (see Eq. (100)), leads to capture. The critical trajectory that corresponds to the dividing line between capture and noncapture is also shown in Fig. 26. It has a pee1-off value close to $v=0.1363$ and would come to rest exactly at the unstable equilibrium configuration corresponding to the saddle point. The other trajectory shown in Fig. 26 is a fission path, corresponding to starting off a very small distance away from the saddle, along the unstable normal mode leading to fission.

Figure 30 is similar to $\mathrm{Fig} .29$ but corresponds to a super-heavy system with $A=300, Z=114$. In attempting to make such a nucleus by the head-on collision of two equal pleces, one is faced with a potential-energy landscape sloping monotonfcally outwards toward disintegration. For all peel-off conditions shown in the figure the trajectories lead to re-separation. Even so, except for the lowest injection velocities, the contact between the two nuclei has been quite intimate and a considerable amount of energy has been dissipated during the dynamical evolution after peel-off. This is illustrated in Table III, which shows that after scission the system has up to about $80 \mathrm{MeV}$ less energy than it had available at the moment of peeling off. The underlying mechanism for energy loss is the one-body wall-plus-window formula, but one should note the role of the collective deformation, represented by the neck growth and collapse, which mediates the conversion of part of the energy of relative motion into internal excitation.

The dynamical trajectorles shown In Figs. 29 and 30 were obtained by a numerical Integration of Eqs. $(64,65)$ using a programable desk calculator. Analytic solutions of these equations may be obtained in the neighborhood of the saddle polnt by linearizing them in the small deviations of $\sigma, v$ from the saddlepoint values $\sigma_{\mathrm{sp}}, \nu_{\mathrm{sp}}$. 
TABLE III. The propertles of the six trajectories in Fig. 30 are listed at the Inttial 1nstant of peeling off from the geometrical boundary and at the last numerically calculated point, near the final collapse of the neck. The last column gives the energy dissipated in the dynamical evolution.

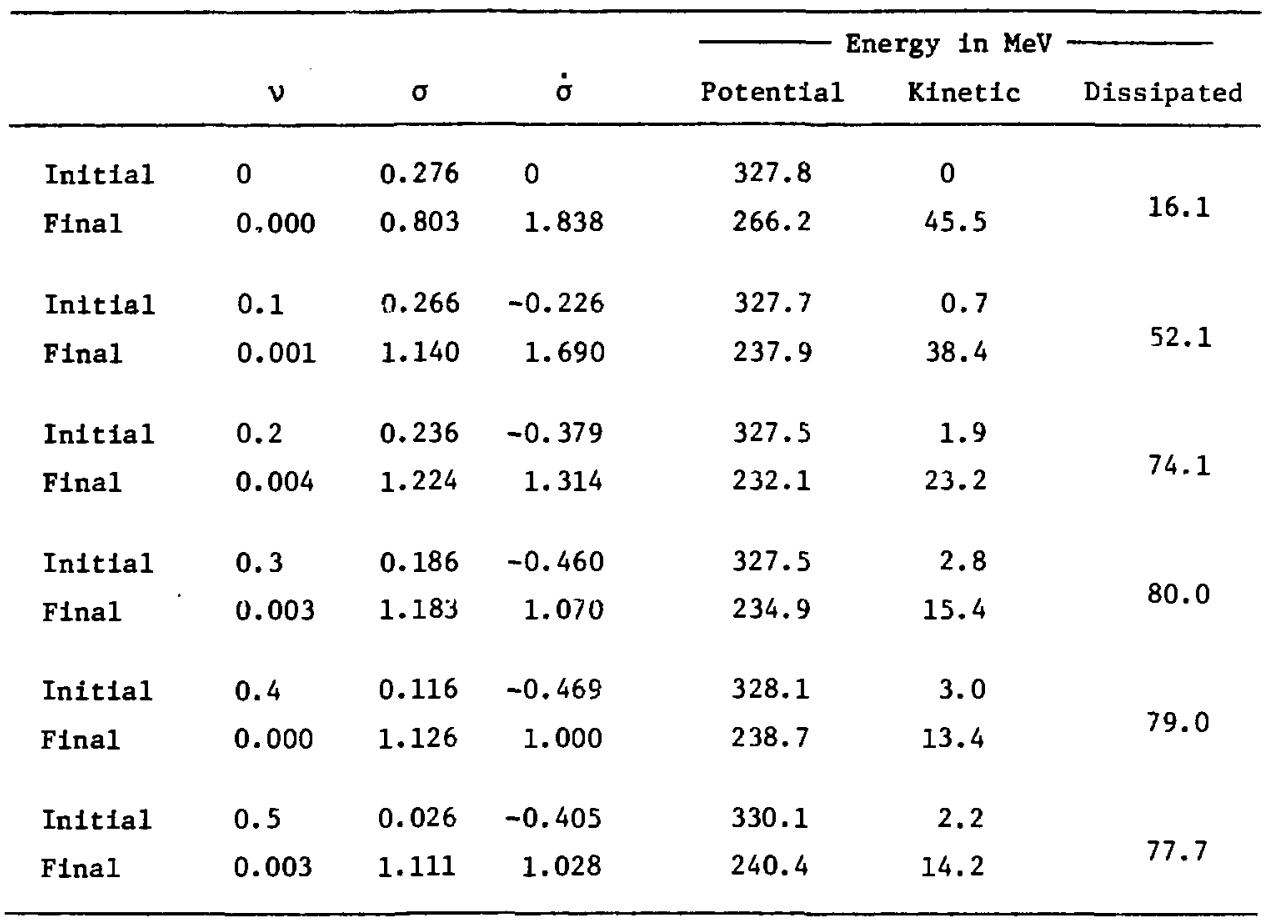

Introducing small varlables

$$
\begin{aligned}
& x \equiv \sigma-\sigma_{s p} \\
& y \equiv v-v_{s p}
\end{aligned}
$$

(not to be confused with the flssility and rotation parameters) the linearized equations of motion take the form

$$
\begin{aligned}
& \ddot{x}+c_{0} \dot{x}=-c_{3} y-c_{4} x \\
& \dot{y}=-c_{2} y-c_{2} x
\end{aligned}
$$

where

$$
\begin{array}{ll}
c_{c}=\nu_{s p}^{2} / \mu \\
c_{1}=\left(3 \nu_{s p}-1+\sigma_{1}\right) / 2 \nu_{s p} \lambda_{s p} \\
c_{2}=1 / 4 \nu_{s p} \lambda_{s p} & \\
c_{3}=\mu^{-1} & \text { for } \sigma_{s p}>0 \\
c_{4}= \begin{cases}2 x / \mu\left(\Lambda+\sigma_{s p}\right)^{3} & \text { for } \sigma_{s p}<0 \\
0 & \end{cases}
\end{array}
$$


Eliminating $y$ between Egs. (101,102) one finds for $x$ a linear, third order differential equation with constant coefficients

$$
\ddot{x}+a_{2} \ddot{x}+a_{1} \dot{x}+a_{0}=0,
$$

where

$$
\begin{aligned}
& a_{0}=-c_{0}-c_{1} \\
& a_{1}=c_{0} c_{1}+c_{4} \\
& a_{2}=c_{0}+c_{1} .
\end{aligned}
$$

The general solution may be written as

$$
x \equiv \sigma-\sigma_{s p}=C e^{-K \tau} \cos (\omega \tau+\delta)+B e^{m \tau},
$$

where $B, C$, and $\delta$ are three arbitrary constants and $K, \omega$, and $m$ are three characteristic frequencies of the motion, given by the following formulae:

$$
\begin{aligned}
& K=\frac{1}{2}\left(s_{1}+s_{2}\right)+\frac{1}{3} a_{2}, \\
& \omega=\frac{\sqrt{3}}{2}\left(s_{1}-s_{2}\right), \\
& m=\left(s_{1}+s_{2}\right)-\frac{1}{3} a_{2},
\end{aligned}
$$

where

$$
\begin{aligned}
& s_{1}=\left[r+\left(q^{3}+r^{2}\right)^{1 / 2}\right]^{1 / 3} \\
& s_{2}=\left[r-\left(q^{3}+r^{2}\right)^{1 / 2}\right]^{1 / 3}
\end{aligned}
$$

with

$$
\begin{aligned}
& r=\frac{1}{6}\left(a_{1} a_{2}-3 a_{0}\right)-\frac{1}{27} a_{2}^{3}, \\
& q=\frac{1}{3} a_{1}-\frac{1}{9} a_{2}^{2},
\end{aligned}
$$

The associated solution for $y$ is given by

$$
\begin{aligned}
y & \equiv v-\nu_{s p}=-\frac{1}{c_{3}}\left(c_{4} x+c_{0} \dot{x}+\ddot{x}\right) \\
& =-\frac{1}{c_{3}}\left\{c e ^ { - K \tau } \left[\left(c_{4}-c_{0} k+k^{2}-\omega^{2}\right) \cos (\omega \tau+\delta)\right.\right. \\
& \left.\left.+\left(-c_{0} \omega+2 K \omega\right) \sin (\omega \tau+\delta)\right]+B e^{m \tau}\left(c_{4}+c_{o} m+m^{2}\right)\right\} .
\end{aligned}
$$


As can be seen from Eqs. $(105,106)$ the general motion near the saddle is a superposition of a damped harmonic oscillation with frequency $w$ and sharacteristic decay time $\mathrm{K}^{-1}$, and an exponential motion away from the saddle with a characteristic growth time $\mathrm{m}^{-1}$. These characteristic times, or rather frequencles, are plotted in Fig. 31 after (multiplication by hut in order to convert them into energies). From the appearance of $\mathrm{hm} / \mathrm{u}_{\mathrm{t}}$, which would be the quantum energy for an oscillator obtained by inverting the fission barrier at the saddle point, we deduce that this barrier is very thin for 11ght nuclei, decreases rapidly with Increasing $A$, and becomes very broad near $A \approx 200$. The quantum frequency $h \omega / u_{t}$ of the elgenmode orthogonal to the fission direction goes down from several MeV for light nuclei to $\sim 1 \frac{1}{2}$ near $A=200$. The damping width $h K / u_{t}$ is more nearly constant and of the order of $2 \frac{1}{2}$ to $1 \frac{1}{2} \mathrm{MeV}$. This Implies that for light nuclei the w-vibration might lead to a definite resonance behavior, less likely for heavier systems where the width becomes comparable to the resonance energy. (It should be borne in mind that all these numerical estimates are at best semi-quantitative, with considerable inaccuracies expected because of the simplified physics and drastically parameterized shape geometry.)

The above are a few examples of current applications of the macroscopic one-lody dissipation dynamics to nucleus-nucleus collisions. This is only the beginning of a systematic investigation but the following qualitative features appear to emerge from such studies.

In the early stages of a collision the asymmetry degree of freedom is inhiblted and the essential degrees of freedom should be the separation and the neck size (or, equivalently, a fragment deformation variable). If the asymetry is frozen completely one finds in configuration space a "conditional saddle point," and dynamical trajectorles divide into two classes, depending on whether they are captured or not on the inside of the saddle. Trajectories that are not captured would include very peripheral (quasi-elastic) collisions but the present dynamical calculations indicate that, (especially for heavier systems), they would also include processes where a considerable fraction of the originally available kinetic energy was dissipated (by an interplay of collectiva deformations and one-bady dissipation). These processes may thus be identified with deep inelastic reactions.

The captured trajectories would be expected to lead to a qualitatively different type of reaction. For nearly symmetric systems, when the conditional saddle is essentially also the true (unconditional) saddle defining the region of configuration space corresponding to a compound nucleus, capture would be synonymous with compound nucleus formation. As the asymmetry grows, however, the conditional saddle deviates more and more from the true saddle. (In addition to being asymmetric it has also a thinner neck because the electric repulsion between the two pleces is less.) The captured trajectorles will then begin to divide into two streams: those that continue to be captured on the inside of the true saddle and lead to compound nucleus formation and those that lead to re-disintegration. The latter fate is possible (even after sapture inside the conditional saddle) but only by virtue of a change in the asymmetry degree of freedom in the direction toward symmetry. Thus a change toward symmetry increases the electric repulsion between the two pieces and a system that, at the original asymmetry, was subjected (after capture inside the conditional saddle) to a compacting driving force, may find this force change sign and become a disintegrating tendency as the system creeps toward symetry. Since a (generally finite) approach toward symmetry is mandatory for the re-disintegration of the system, one expects such reactions to be characterized by a relatively longer time scale than deep inelastic reactions and to be in other respects (e.g., asymetry) qualitatively different. Since, however, capture into a compound nucleus is not involved, these "composite nucleus" reactions should be characterized by times much shorter than compound nucleus reactions. 
Thus a new type of reaction is suggested on theoretical grounds by the notion of conditional saddle point shapes at $f$ ixed asymetry and by the fact that with growing asymetry a large region of configuration space opens up (for heavy systems) between the conditional saddle and the true saddle. These composite nucleus reactions, which are captured inside the conditional saddle but not inside the true saddle, should be characterized by time scales intermediate between deep inelastic and compound nucleus times and should have mass and angular distributtons approximately, but only approximately, similar to compound nucleus reactions. They may be tentatively identified with the "quasi-fission" reactions observed experimentally.

When the asymmetry of the two colliding nuclei is chosen sufficiently large the composite nucleus reactions will often lisappear, because a new feature comes into evidence. Thus, as mentioned earlier, the driving force in the asymetry degree of freedom will (for not too light systems) change sign if the asymmetry is sufficiently large. Such systems will, after capture inside the conditional saddle, experience a driving force both towards smaller separations and larger asymmetries. The configuration will, on both accounts, tend to become more compact and a compound nucleus, if it exists at all, is certain to be formed. (Sufficient1y asymetric reactions, if they could be arranged in practice, would thus provide ideal paths toward exploring the existence of super-heavy nuclei.) If a compound nucleus does not exist then even the very asymmetric reactions would continue to exhibit the properties of composite nucleus reactions with intermediate time scales of the order of the characteristic creep times mentioned in Lecture II.

Summary

In these lectures I presented an attempt to set up a theory of the dynamics of nuclear shape changes, for small (sub-sonic) collective velocitles.

To set up the equations of motion one needs three forces: conservative, dissipative, and inertial.

The first lecture dealt with conservative forces and stressed the division of the underlying potential energy into I ocal, Proximity, and Global cerms.

The second lecture suggested a similar classification for the dissipative forces and went on to consider the Local part. The underlying physirs was the approximate validity of the Independent-Particle model. This, combined with the Randomization Hypothesis, led to simple dissipation formulae which suggested that dissipative forces may often overshadow the inertial forces.

The third lecture set up particularly simple equations of motion and explored some of the consequences. It is still too early to say how useful the very simple versions of the One-Body Dissipation theory will turn out to be. One should continue testing and refining the theory on three levels: from first principles, by comparisons with nunerfcal studies (e.g., Time-Dependent Hartree-Fock calculations) and by comparisons with (crucial) experiments.

\section{References}

[1] J.BLOCKI et al., Ann. Phys. 105, 427 (1977).

[2] H.DELAGRANGE, L.C.VAZ and J. M.ALEXANDER, "Optical Model Analysis for ${ }^{16} 0+$ ${ }^{208} \mathrm{~Pb}$ : Evidence for Dynamic Shape or Density Changes," Stonybrook preprint, (April 1979, submitted for publication); G.DELIC et al., Phys. Letters 69B, 20 (1977).

[3] CHIN-FU TSANG, "On the Macroscopic Aspects of Nuclear Structure with Applications to Super-Heavy Nucle1," Ph.D. Thesis, University of California, Lawrence Radiation Laboratory Report UCRL-18899 (May 1969). 
[4] W.D. rYERS in "Dynamic Structure of Nuclear States," Proc. of the 1971 Mont Tremblant Int. Summer School (Univ. of Toronto Press, 1972) and Lawrence Berkeley Laboratory preprint LBL-209 (July 1971).

[5] A.BOHR and B.R.MOTTELSON, "Nuclear Structure" (W.A.Benjamin Inc., 1975) Vol. II, P. 222.

[6] M. BRACK et al., Rev. Mod. Phys. 44, 320 (1972).

[7] Ref. 5, Vol. II, p.578.

[8] J.BLOCKI et al., Ann. Phys. 113, 330 (1978).

[9] J.RANDRUP and W.J.SWIATECKI, "One-Body Dissipation and Nuclear Dynamics," NORDITA preprint NORDITA-78/38 (July 1978, submitted to Annals of Physics).

[10] W.D.MYERS, "Droplet Model of Atomic Nuclef," (IFI/Plenum, 1977).

[11] W.J.SWIATECKI, Phys. Rev. 104, 993 (1956).

[12] Ref. 5, Vol. II, p.654.

[13] J.R.BIRKELIND et al., Phys. Rev. Lett. 40, 1123 (1978).

[14] J.R.BIRKELUND et al., "Heavy Ion Fusion: Comparison of Experimental Data with Classical Trajectory Models," Univ. of Rochester preprint UR-NSRL-193 (May 1979, submitted to Physics Reports).

[15] J.R.NIX and A.J.SIERK, private communication 1979, to be published.

[16] S.COHEN and W.J.SWIATECKI, Ann. Physics 19, 67 (1962); 22, 406 (1963). W.J.SWIATECKI, Jour. de Physique, Supp1. $\overline{8}-9$, Vol. 33 (1972), p.C5.

[17] C.COHEN, F.PLASIL, and W.J.SWIATECKI, Ann. Physics $\overline{82}, 557$ (1974), esp. p.562.

[18] M.ABRAMOWITZ and I.A.STEGUN, "Handbook of Mathematical Functions," (U.S. Dept. of Commerce, 1964), p.446. 

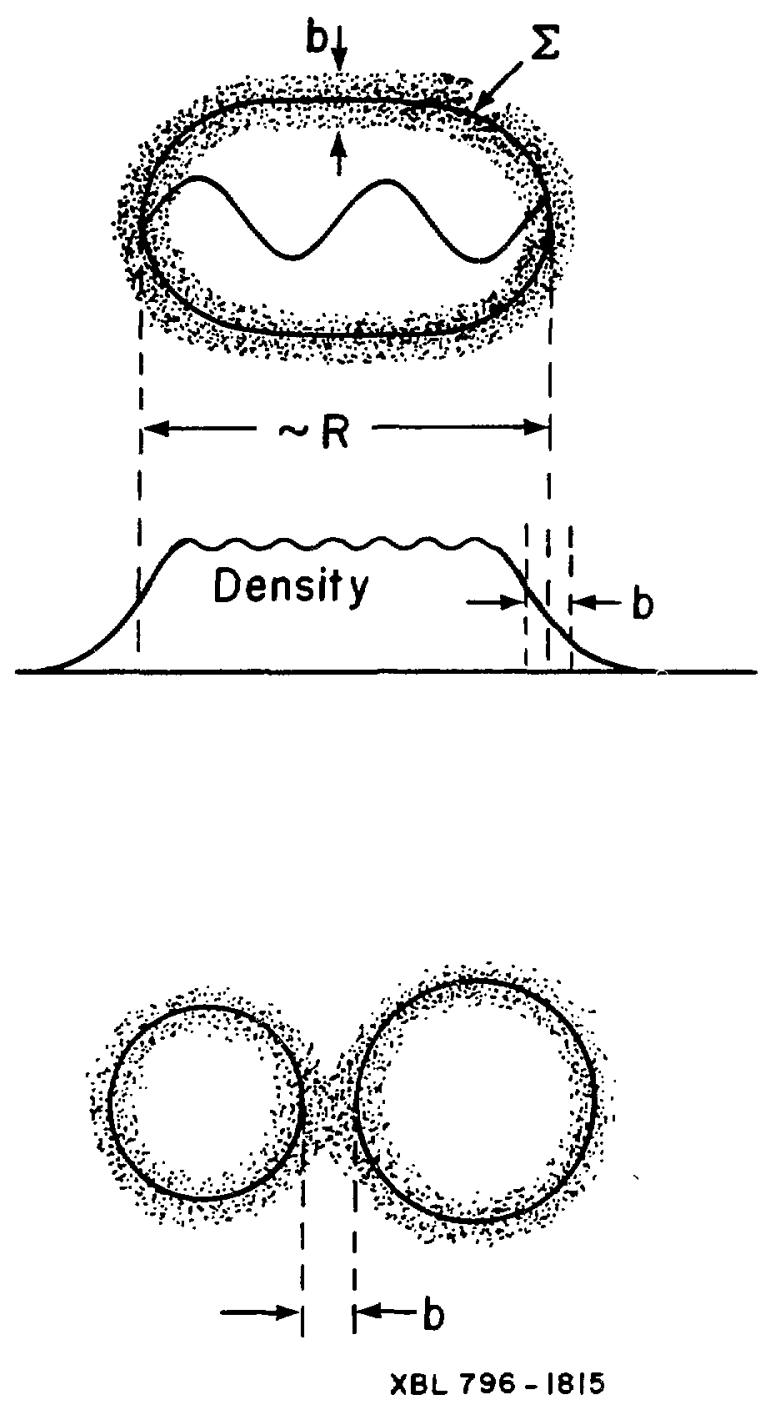

Fig. 1. A leptodermous blob $\Sigma$, whose elements interact by forces of range $\sim b$, and filled with wavefunctions that feel out distances of order $\sim R$. 


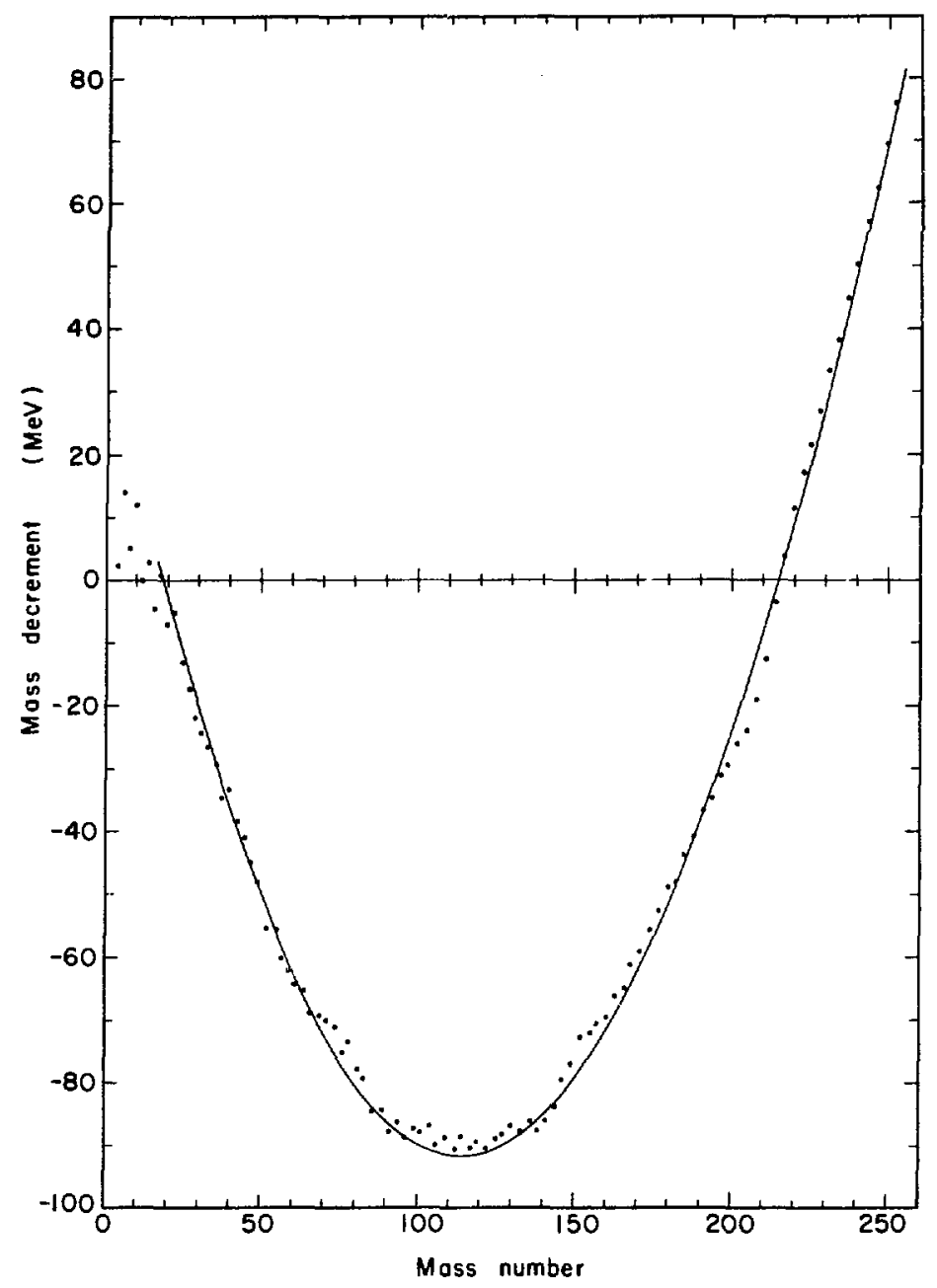

UUB 6567

Figure 2. The mass decrements (closely related to nuclear binding energles) are plotted for 97 beta-stable nuclet. The curve Is a liquid-drop fit based on the "local" part of the potential-energy expression. The deviations are due mostly to shell effects. 


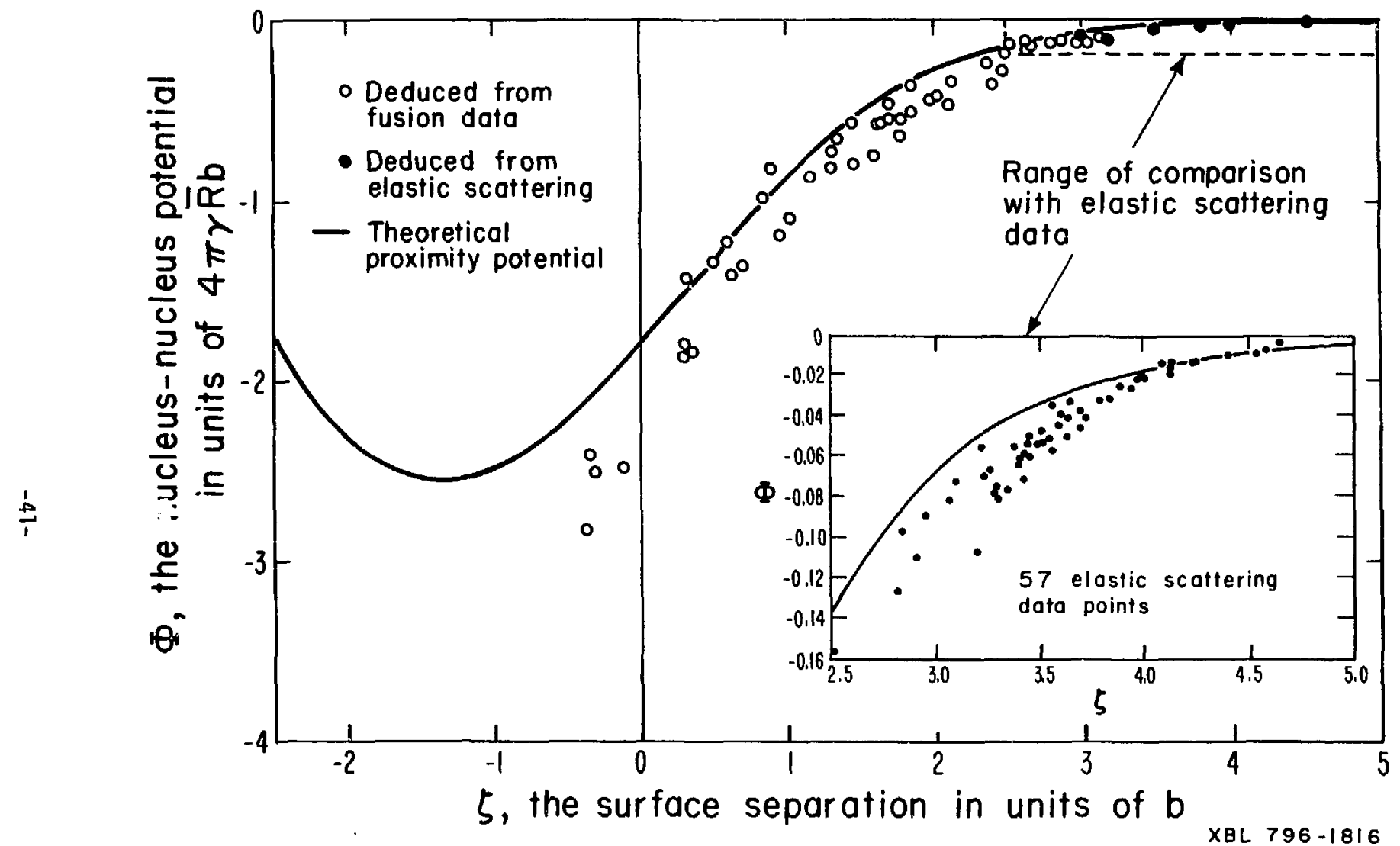

Fig. 3. The Theoretical Proximity interaction potential between nuclei is shown as a function of surface separation. The experimental depths are deduced from elastic scattering and heavy-ion fusion data. The unit on the abscissa corresponds to about $1 \mathrm{fm}$. The range of potential depths displayed goes from a fraction of an Mev to several tens of $\mathrm{Mev}$. 

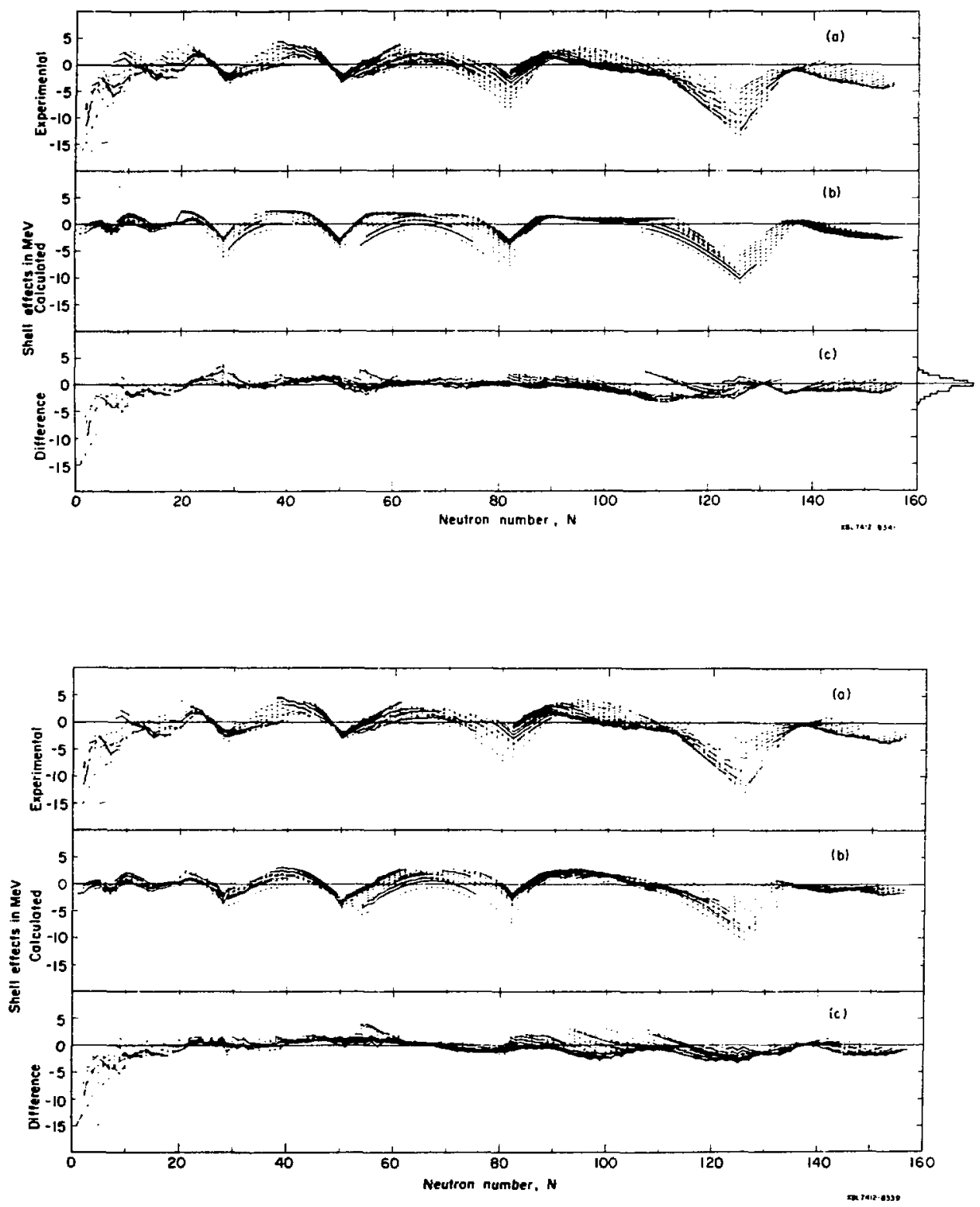

Figure 4. The shell correction to nuclear binding energies (1.e., the experimental mass minus a droplet model $\mathrm{fit}$ ) is displayed as a function of neutron number [1ine (a)]. Line (b) is a theoretIcally calculated ohell correction, using the Strutinsky shel1correction method in the lower part and a schematic model of bunched levels in the upper part. Iine (c) is the remaining deviation. See Ref. 10 . 
(a)

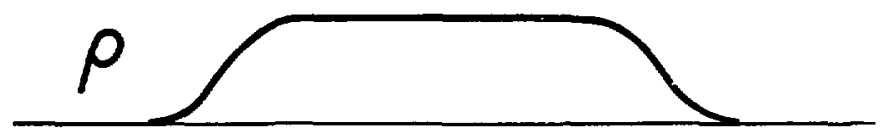

(b)
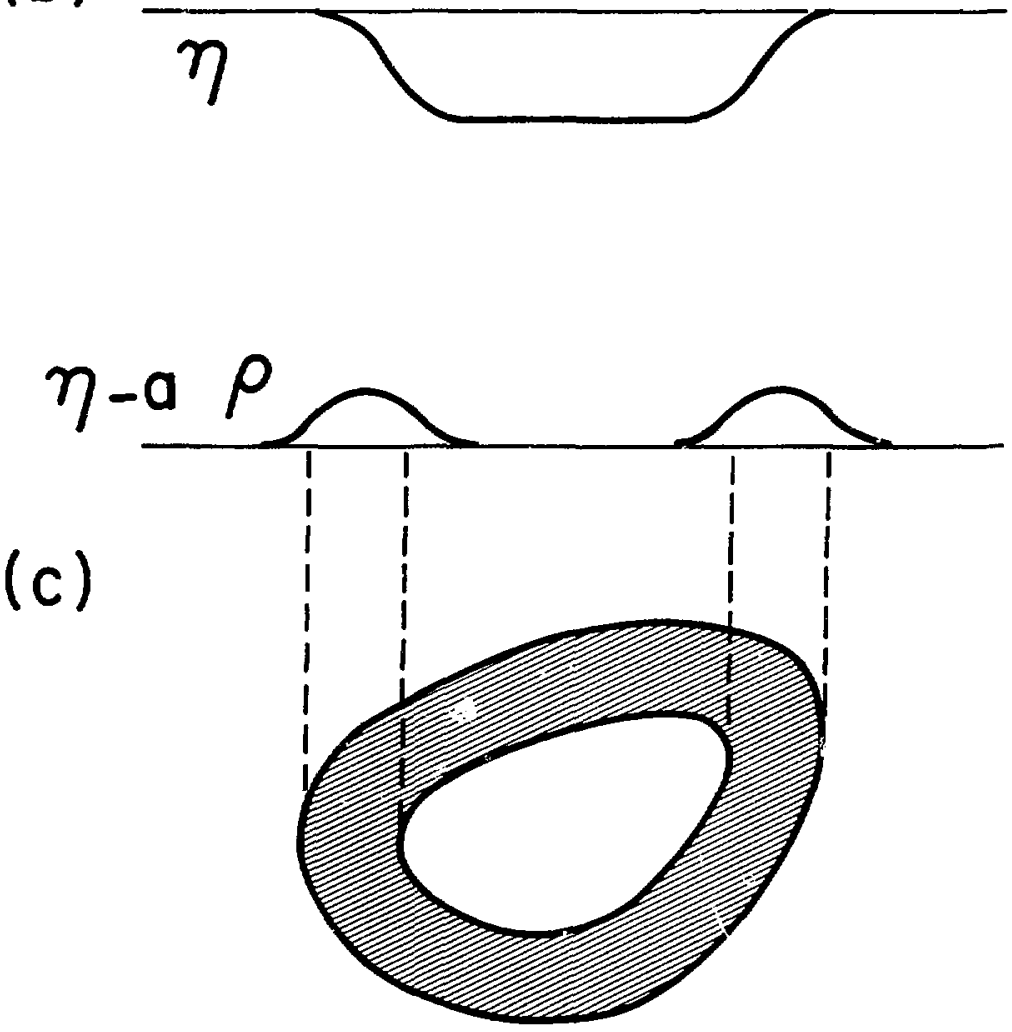

XBL 763-2575

Figure 5. A schematic 1llustration of the particle density $p$, energy density $\eta$, and the surface-energy bump function $n$-a $\rho$ for a leptodermous system. 


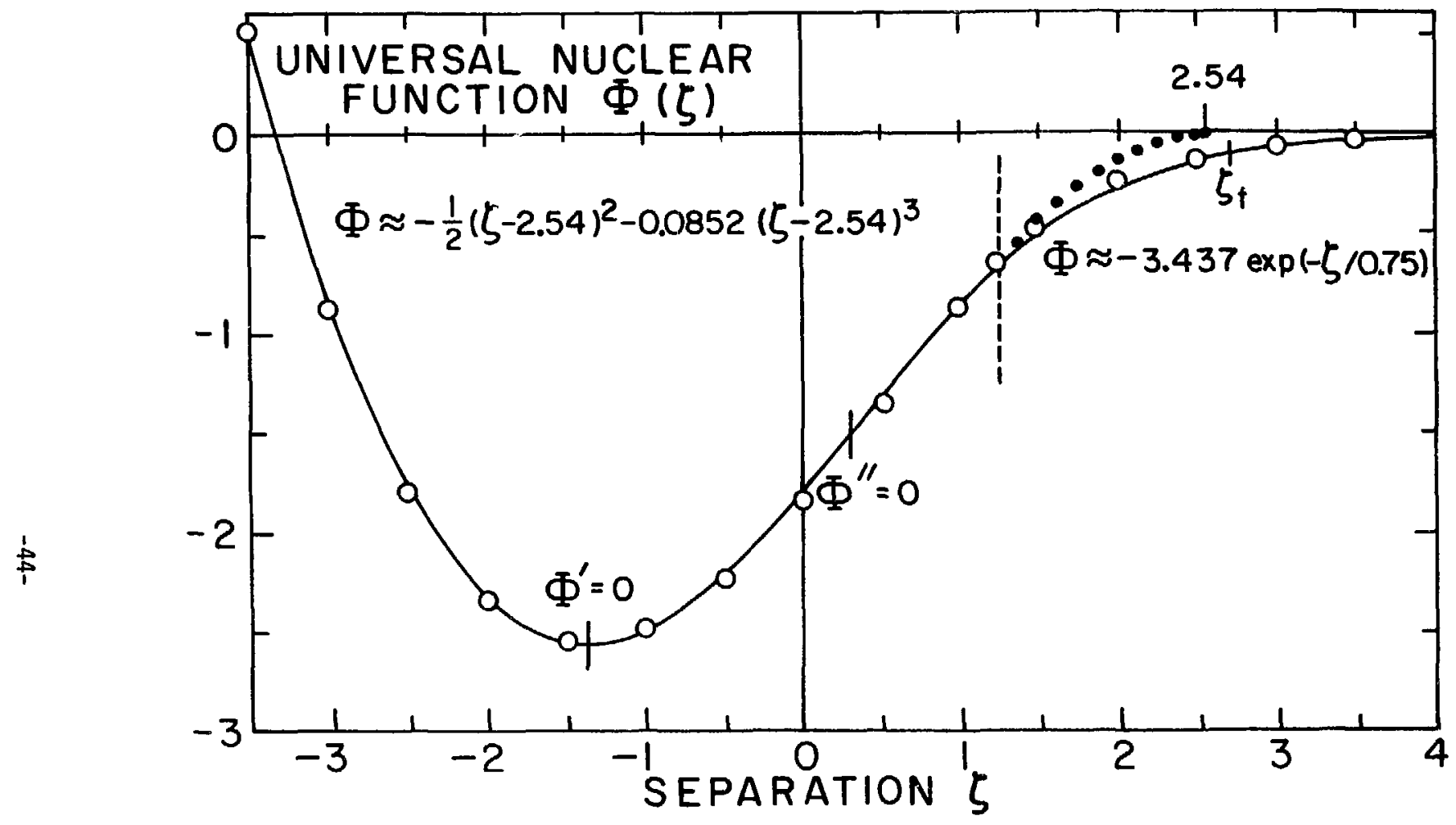

XBL759- 4073

Figure 6. The universal nuclear Proximity Potential Function, $\Phi(\zeta)$, and the cubic-exponential approximation (circles). The dots continue the cubic part to zero at $\zeta=2.54$. The frozen Thomas-Fermi density distributions on which this calculation of $\Phi$ is based touch at the separation $\zeta_{t}{ }^{\circ}$ 


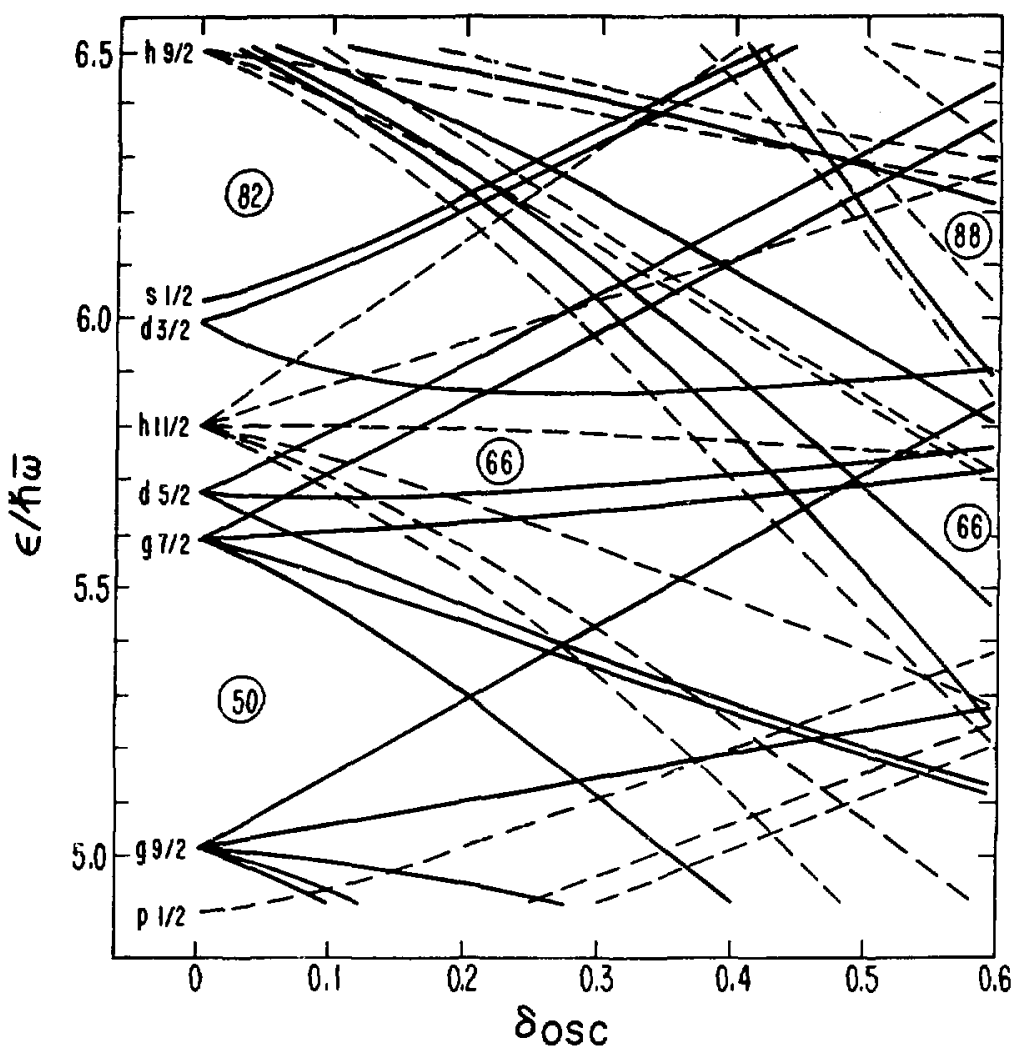

XBL $796-1817$

Fig. 7. A portion of a N1lsson level diagram for protons, showing the elgenvalues in a modified oscillator potential with spin-orbit coupling. The potential well is spherical on the left and prolate-spheroidal towards the right. The proton numbers are circled. See Ref. 5 . 


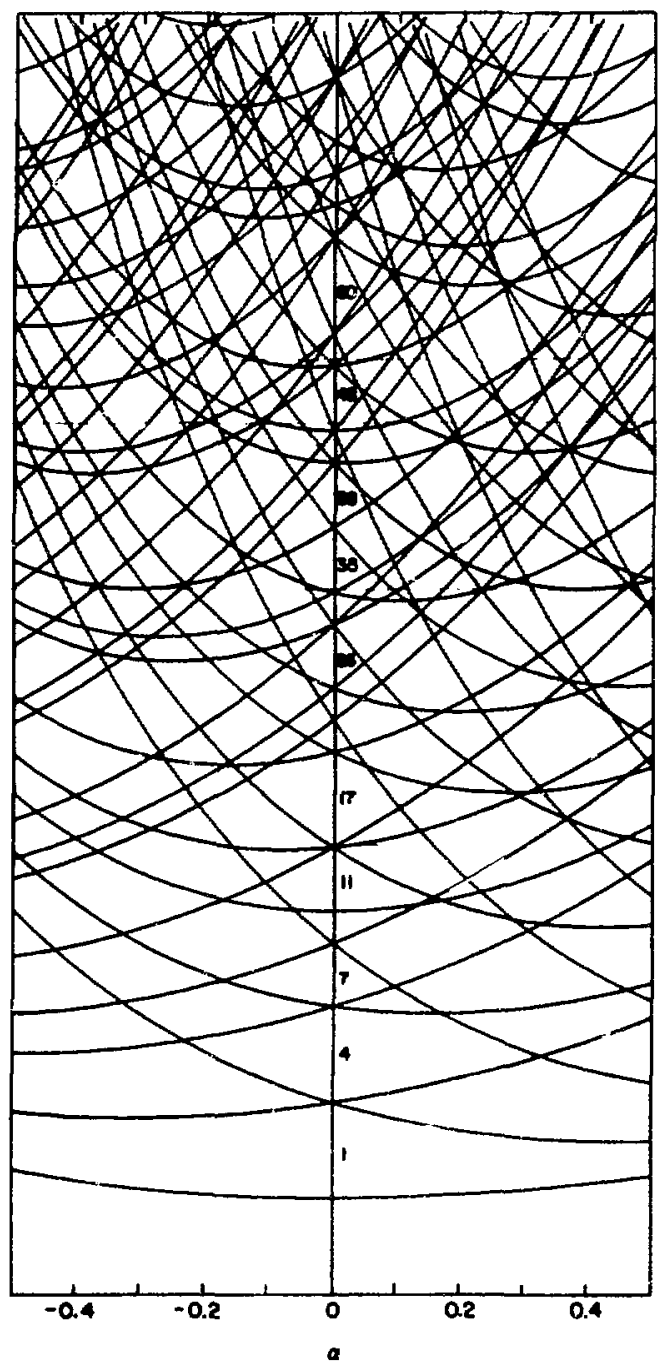

xesos- 28es

Figure 8. A Nilsson diagram for a Hill-Wheeler box. The box is a cube in the middle, oblate on the left, and prolate on the right (from Ref. 3). The numbers of eigenvalues (of ten degenerate) are indicated along the ordinate. With two neutrons and two protons per elgenvalte the number 60 would represent a system with $A=240$. 


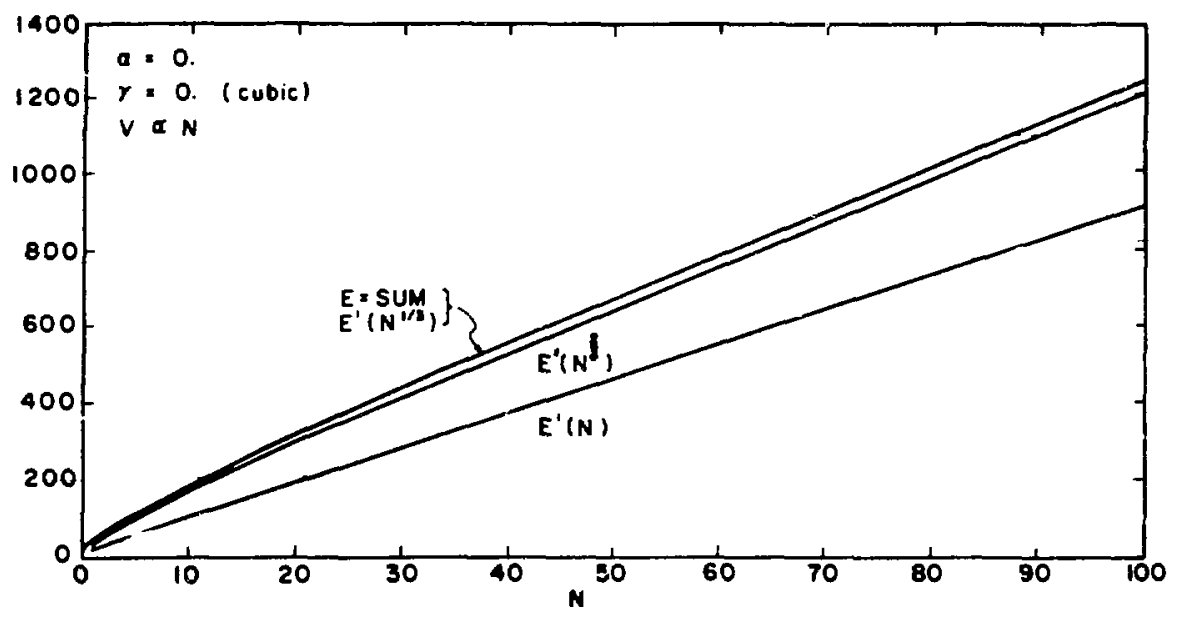

thes-2ise

Figure 9. Comparison of the sum over eigenvalues (labeled $E=S U M$ ) for a cubic H1ll-Wheeler box, with "local" series expansions taken to order $N, N^{2 / 3}$, and $N^{1 / 3}$ in the number of eigenstates $N$. The ordinate is in units that correspond roughly to MeV. 


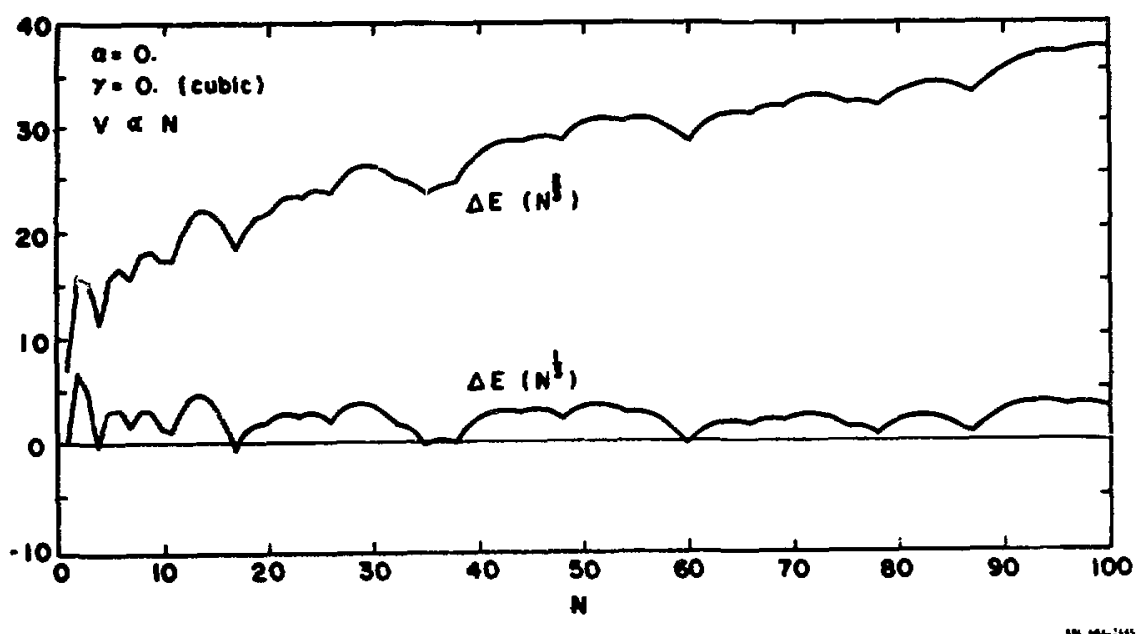

Figure 10. The differenca between the sum over eigenvalues and local expansions taken to order $\mathrm{N}^{2 / 3}$ (upper curve) and $\mathrm{N}^{1 / 3}$ (lower curve) are shown for the same box as in Fig. 9. The scale is vastly expanded and shell effects are discernible (e.g., a magic number at $\mathrm{N}=60$ ).
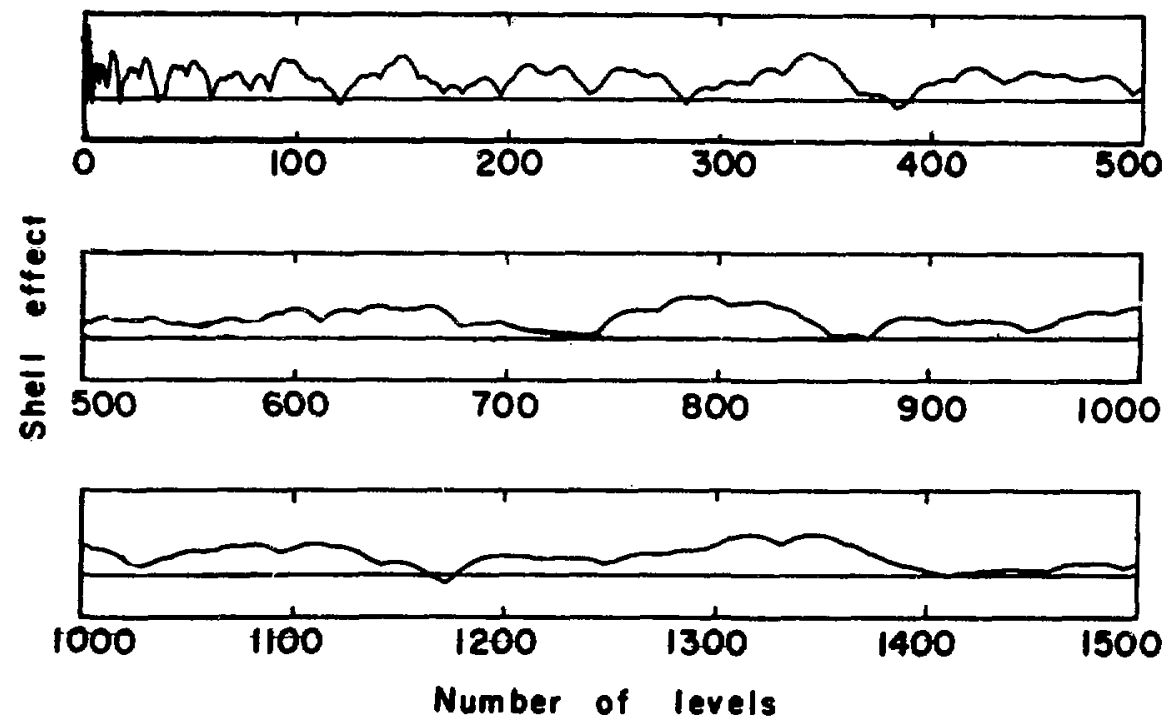

noเ

Figure 11. This is like the lower curve in Fig. 10 but with $\mathrm{N}$ extended to 1500 (i.e., $A=4 N=6000)$. The plot 11lustrates the correctness of the enalytic expansion to order $\mathrm{N}^{173}$. The remalning deviations eppear, Indeed, to be made up of a constant contribution (of order $\left.\mathrm{N}^{\circ}\right)$ and an oscillating shell correction whose amplitude does not seem to vary significantly with $\mathrm{N}$. 


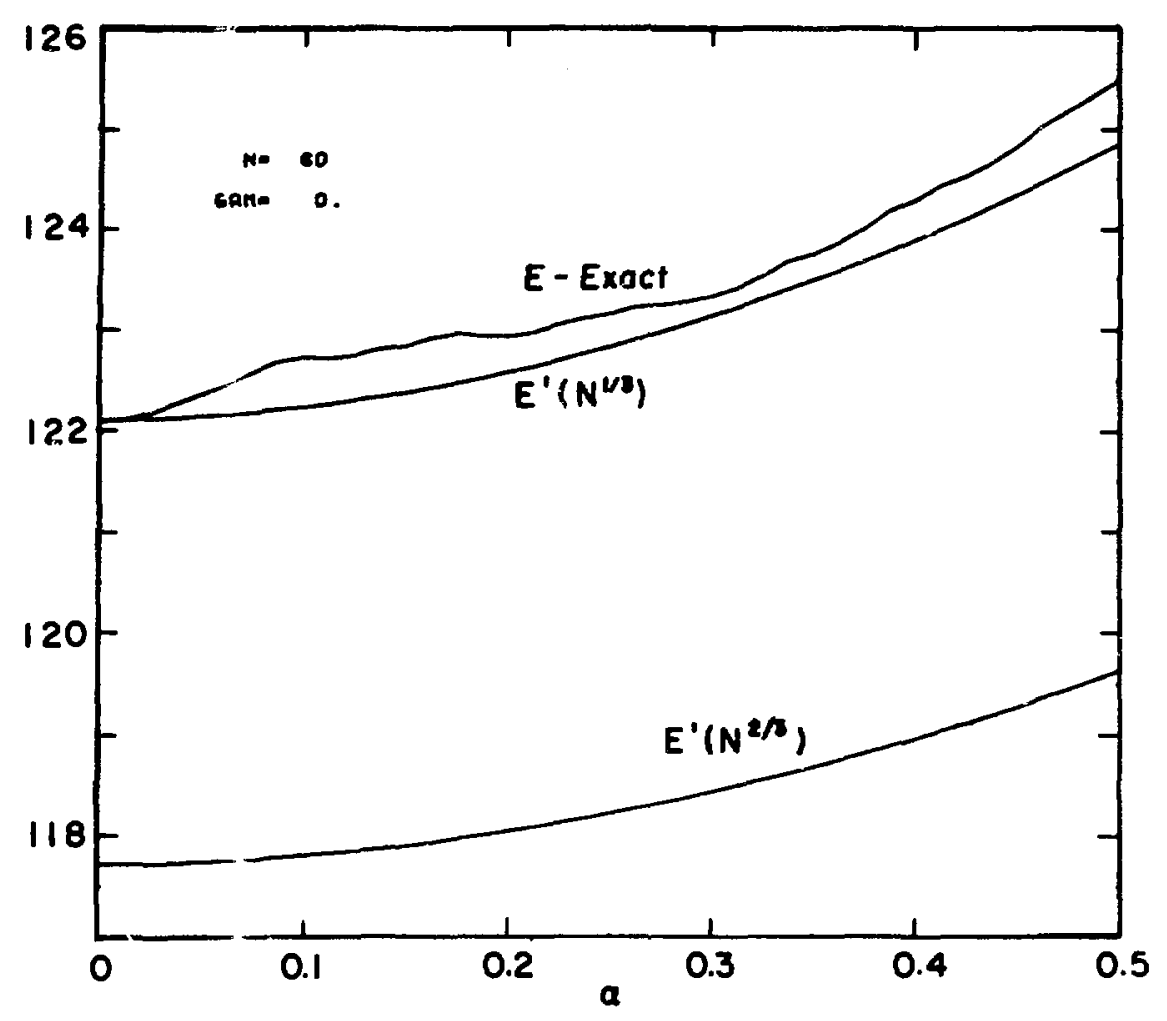

X8L65-2276

Figure 12. In this figure the Hill-Wheeler box with $\mathrm{N}=60$ was def rmed into a prolate box with sides $a, b, c$ in the ratios $a / c=b / c=e^{-3 \alpha / 2}$. (The untt on the ordinate is not the same as in Figs. 9-11.) The flgure shows that the deformability of a given box, as given by the sum over efgenvalues, is also reproduced to within the antictpated accuracy by the local expanstons (to order $N^{2 / 3}$ or $N^{1 / 3}$ ). 


\section{The Wall Formula}

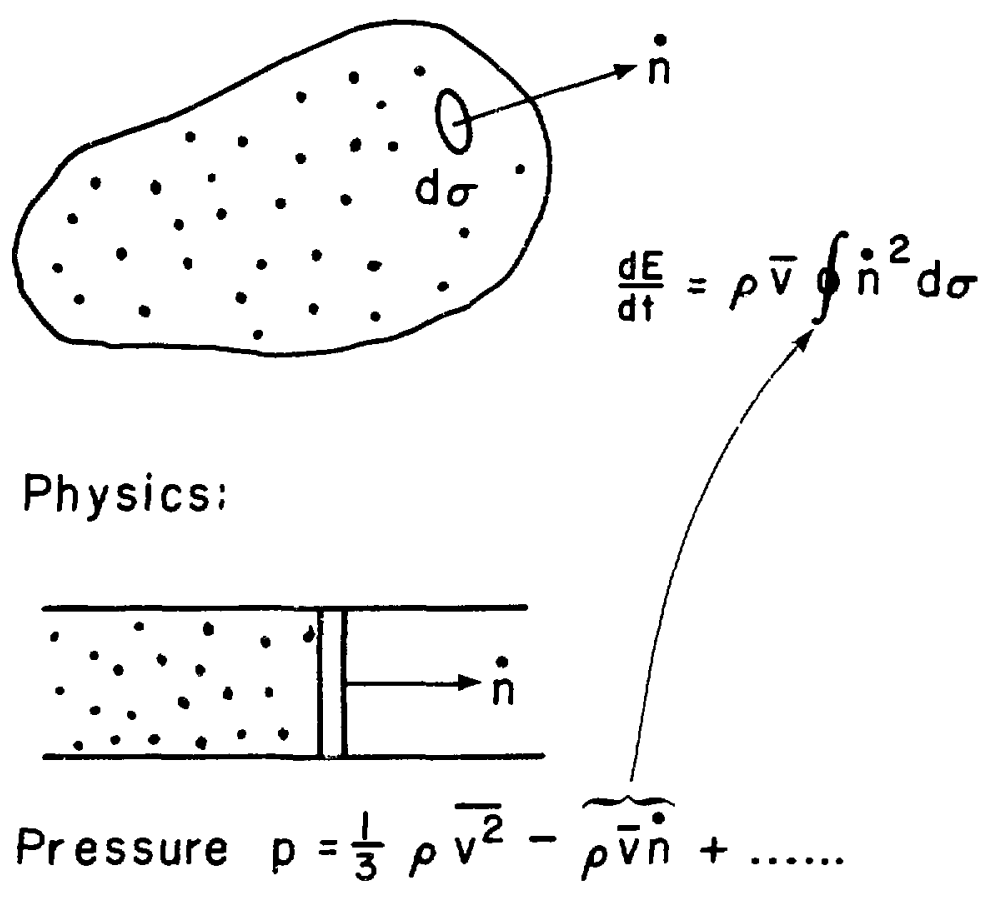

XBL $796-1819$

Fig. 13. The essence of the Wall Formula: a container is filled with a gas of long-mean-free-path particles and its surface elements are deforming with normal speeds specified by $\dot{r}$. The pressure on each element of surface is assumed to be that experienced by a piston moving with respect to an infinitely long cylinder filled with the gas. 


\section{The Window Formula}

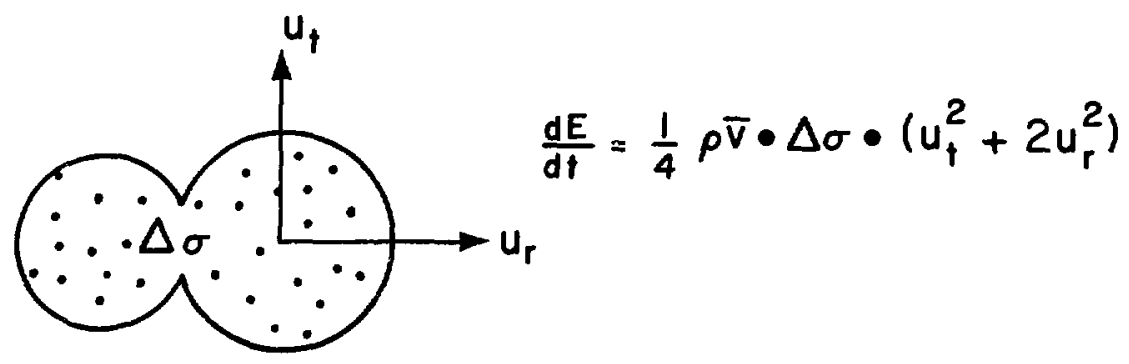

Physics:

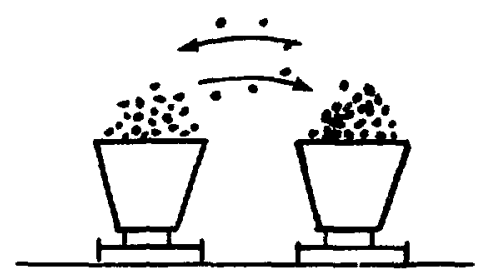

Flux of cool damps out relative motion of trains

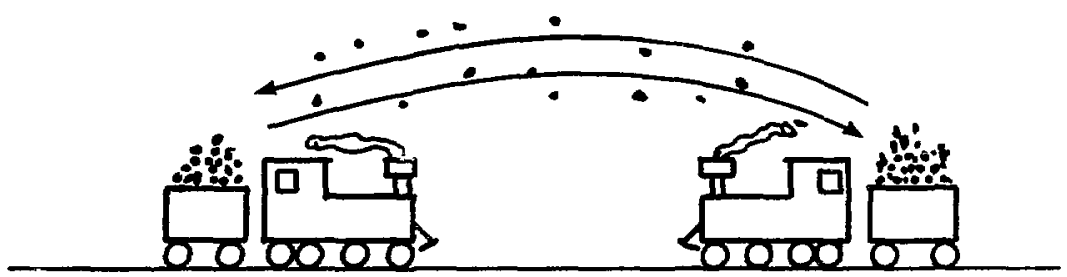

What to do if the brakes fail.

XBL $796-1818$

Fig. 14. The essence of the Window Formula: two vessels are in relative motion and comounicate through a window of area $\Delta \sigma$. The flux of particles between the vessels induces a drag between them. The same drag would tend to reduce the relative speed of two coal trains on parallel tracks if the coal were shoveled back and forth. Or it could be made to slow down two trains on a collision course. 


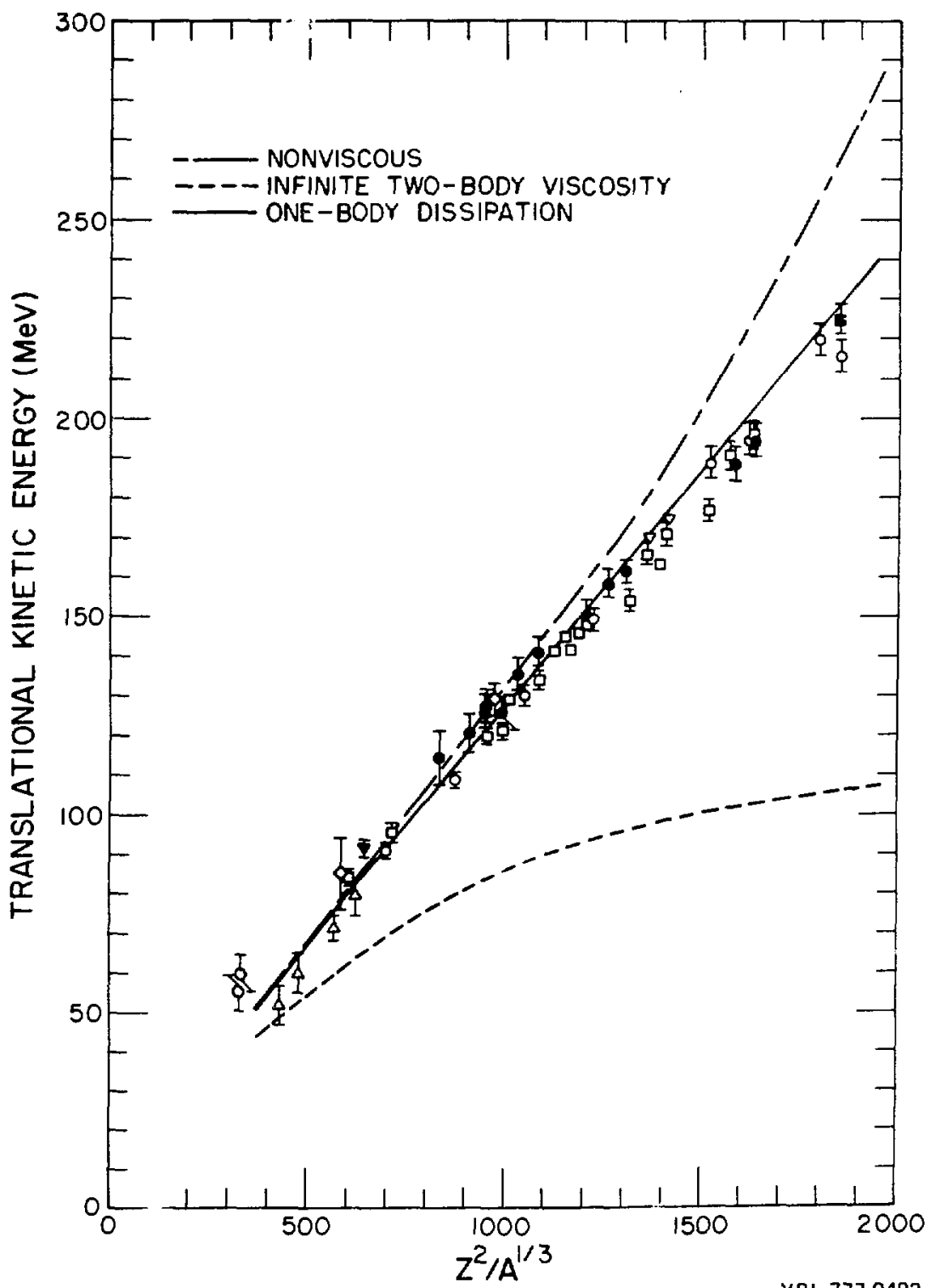

XBL 777.9492

Figure 15. Comparison of calculated and experimental most probable fission fragment kinetic energies as a function of $z^{2} / A^{1 / 3}$. The kinetic energies calculated for nonviscous flow are given by the dot-dashed curve. The dashed curve shows the results for very large two-body viscosity, and the solid curve shows the results for the one-body dissipation considered here. The experimental data are for cases in which the most probable mass division is into two equal fragments; the open symbols represent values for equal mass divisions only and the solid symbols represent values averaged over all mass divistons. 


\section{INFINITE}

TWO-BODY

VISCOSITY

${ }^{130} \mathrm{Cs}, Z^{2} / A=23$
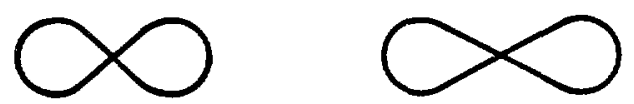

${ }^{184} W, \quad Z^{2} / A=30$
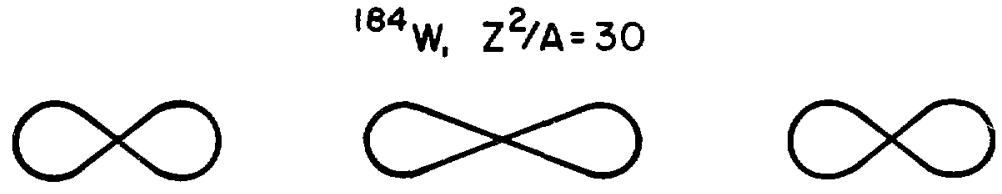

${ }^{236} U, Z^{2} / A=35.9$
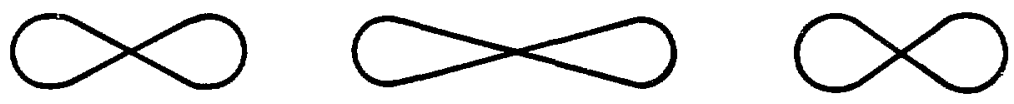

$$
{ }^{288} 110, Z^{2} / A=42
$$
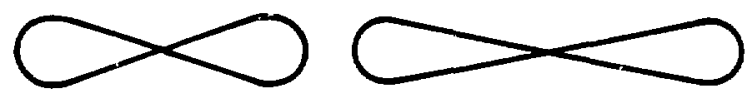

ONE-BODY

DISSIPATION

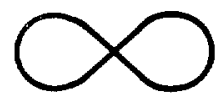

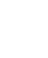




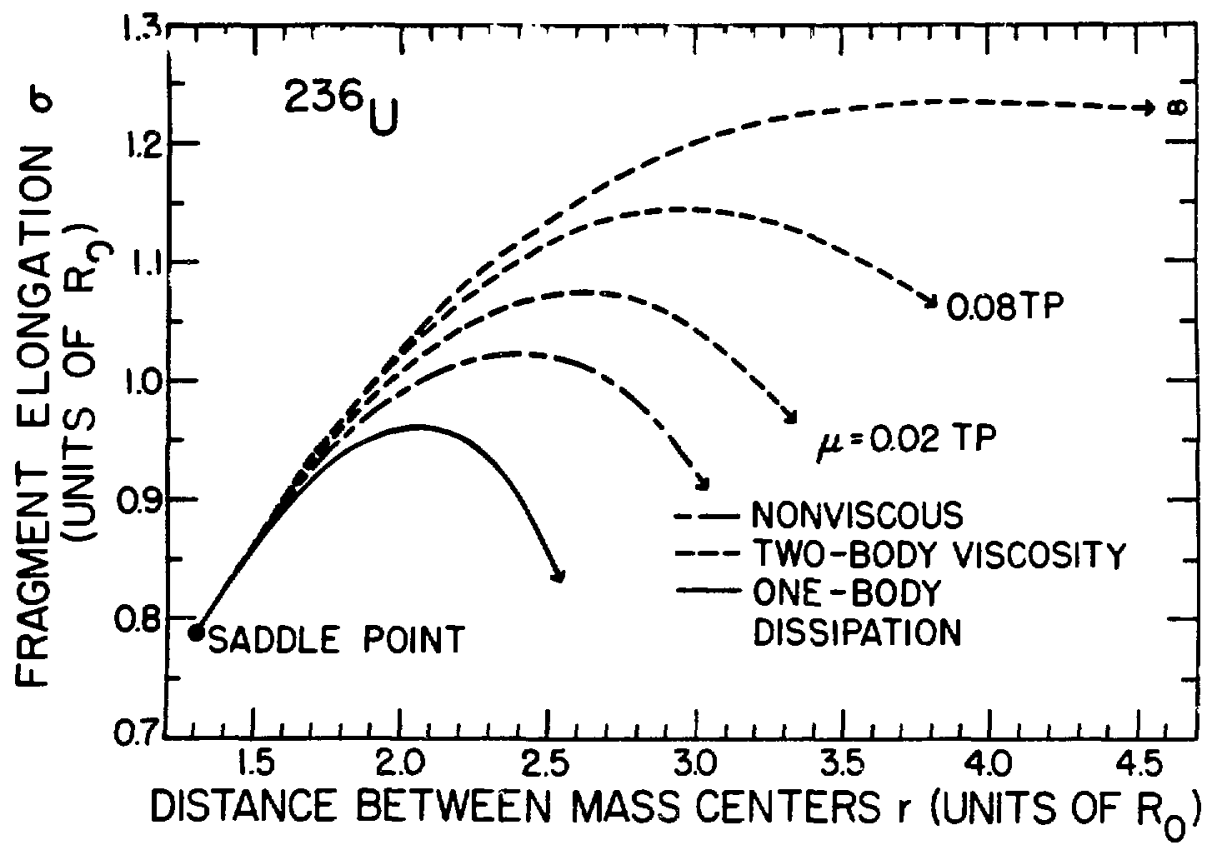

XBL 777.9490

Figure 17. Dynamical paths in $r-\sigma$ space of a ${ }^{236} U$ nucleus from its saddle point to scission. In this figure $r$ is the distance between the mass centers of the fragments, $\sigma$ is a measure of fragment elongation and $R_{o}$ is the radius of the original spherical nucleus (for detalls see Ref. 8 ). The reference path for nonviscous flow is given by the dot-dashed curve. The dashed curves show the paths calculated for various values of the two-body viscosity coefficient $\mu$, which is measured in units of terapoise ( 1 TP $\left.=10^{12} \mathrm{dyn} \mathrm{sec} / \mathrm{cm}^{2}=6.24 \times 10^{22} \mathrm{MeV} \mathrm{sec} / \mathrm{fm}^{9}\right)$. The solid curve shows the path for the one-body dissipation considered here. The scission points are indicated by the tips of the arrowheads. 


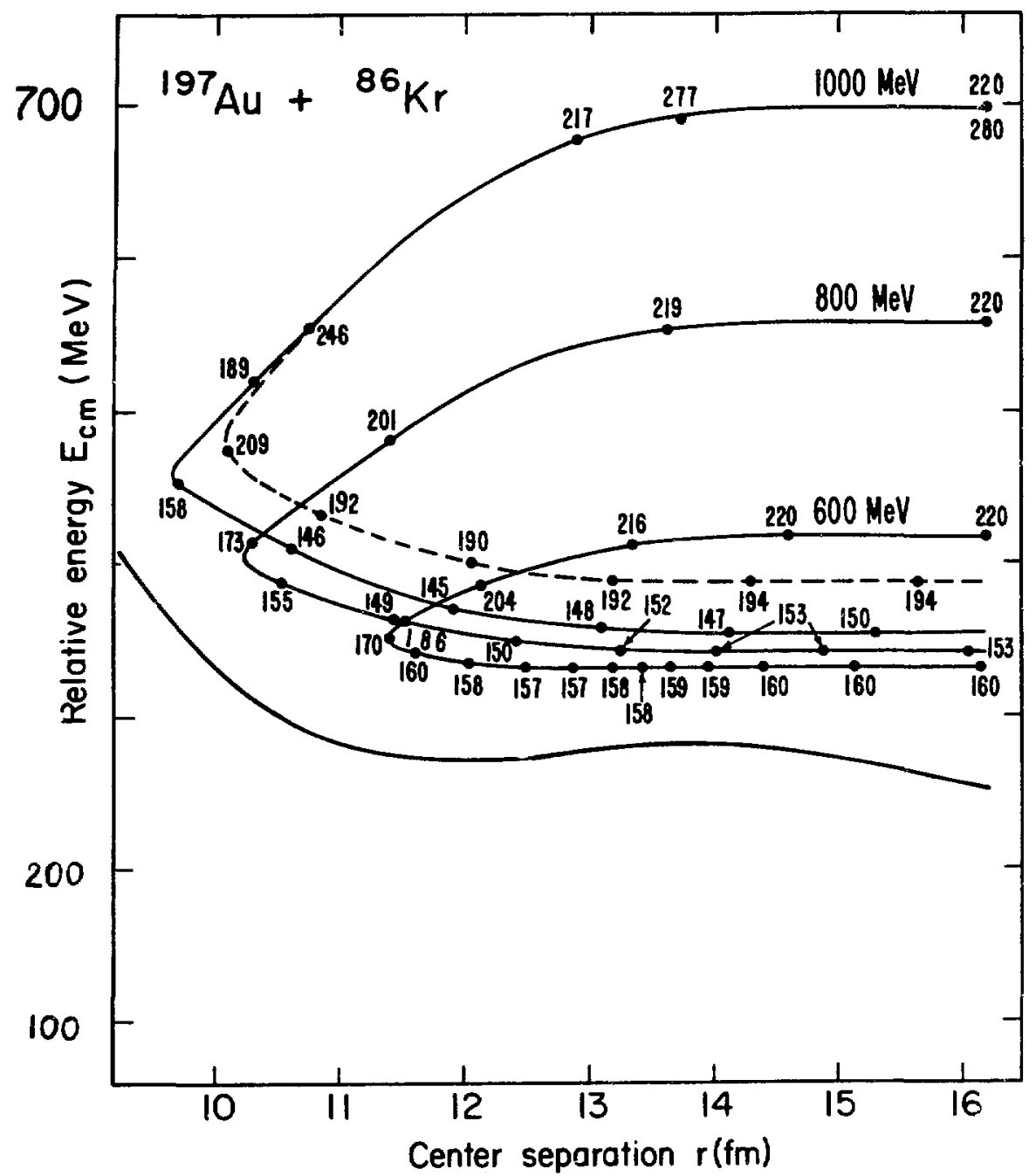

XBL777-1324

Figure 18. Relative center of mass energy $E_{C M}$ vs. center separation for four collisions of ${ }^{86} \mathrm{Kr}$ on ${ }^{197} \mathrm{Au}\left(\mathrm{E}_{1 \mathrm{lab}}=600 \mathrm{MeV}, \ell_{\perp}=220 \mathrm{~h}\right.$; $\mathrm{E}_{\mathrm{lab}}=800 \mathrm{MeV}, \ell_{1}=220 \mathrm{\hbar} ; \mathrm{E}_{1 \mathrm{ab}}=1000 \mathrm{MeV}, \ell_{1}=220$ and $\left.280 \mathrm{~h}.\right)$ The time evolution of the collisions is indicated by dots giving the position at intervals of $10^{-22} \mathrm{sec}$ and the labels on the dots refer to the current orbital angular momentum. The upper part of the curve for $E_{1 a b}=1000 \mathrm{MeV}$ has two sets of dots, one for the trajectory starting with $\ell_{1}=220 \mathrm{~h}$ and the other with $\ell_{1}=280 \mathrm{~h}$. (The trajectories are almost identical at first.) Note that the approach of the orbital angular momentum to its asymptotic value Is not quite monotonic. The lower curve is the interaction energy between the two nuclei. 


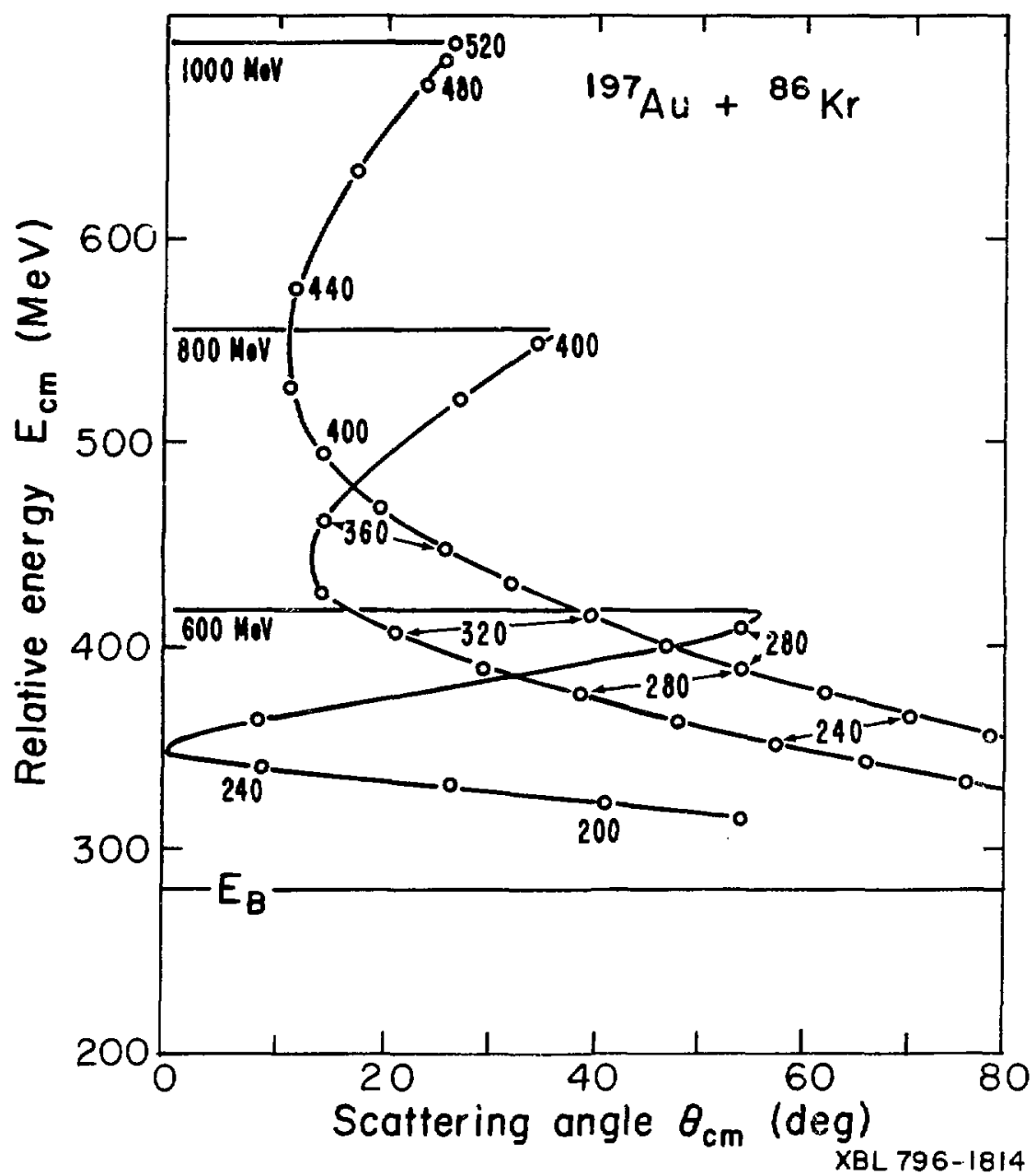

Fig. 19. Energy vs. angle plots (Wilczynski diagrams) for the 1dealized collision of $86 \mathrm{Kr}$ on $197_{\mathrm{Au}}$ at three (lab) energies. The labels on the circled points give the final orbital angular momentum appropriate to the angle and energy indicated. The interaction barrier $\mathrm{E}_{\mathrm{B}}$ (the same, in the model used, for the entrance and exit channels) is indicated. 


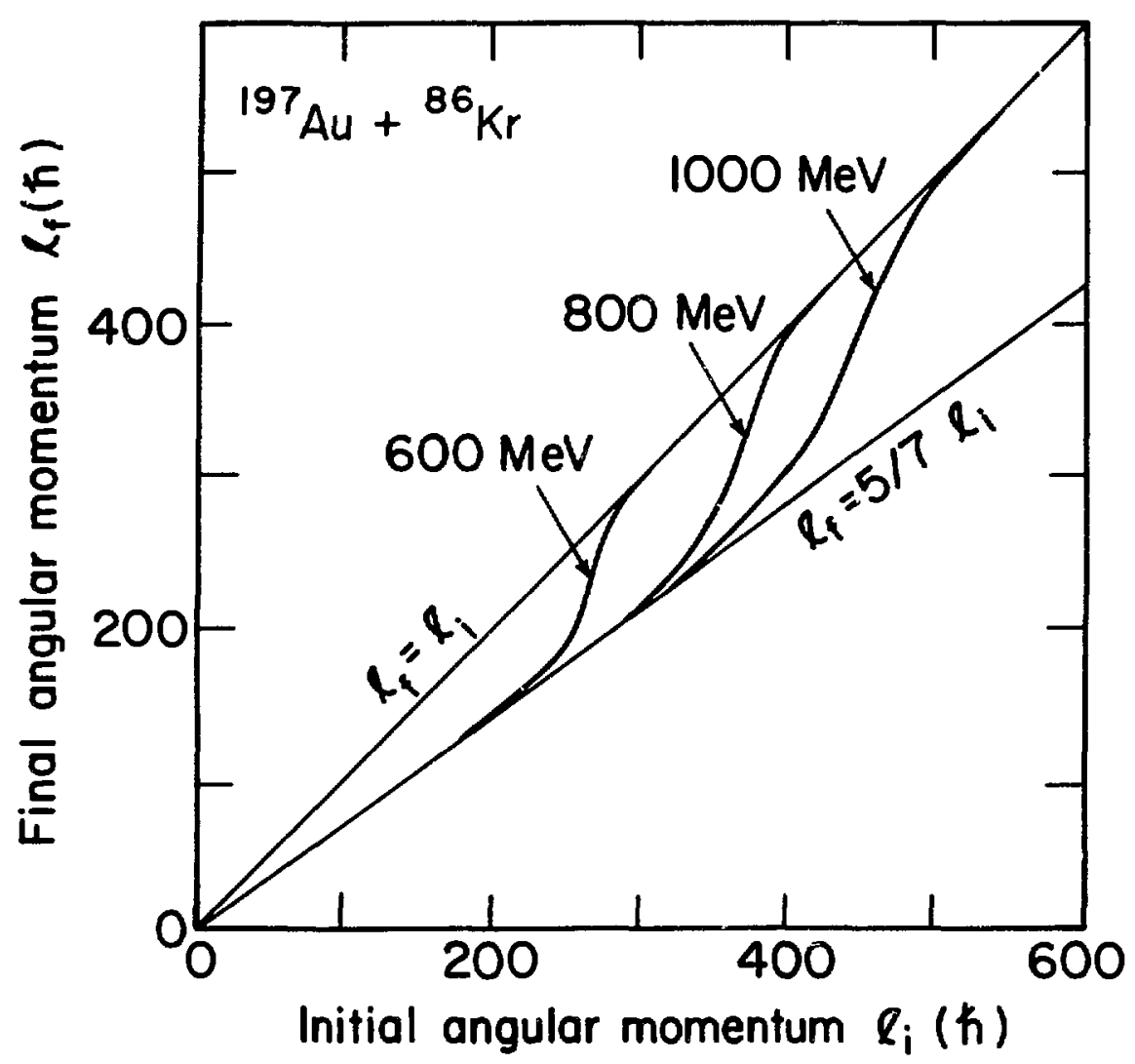

XBL 777-1322

Figure 20. The final orbital angular momentum as a function of the Initial angular momentum for an idealized $86_{\mathrm{Kr}}$ nucleus bombarding an Idealized $19 \mathrm{Au}$ nucleus at laboratory energies of 600,800 and $1000 \mathrm{MeV}$. The window formula, in the form of the proximity friction, was used to describe the dissipation of energy. The value $l_{f}=(5 / 7) l_{1}$ corresponds to the rolling condition which, within the limitations of the model, corresponds to total relaxation in the relative angular degree of freedom. A large part of the cross-section is seen to correspond to such a relaxed situation. 


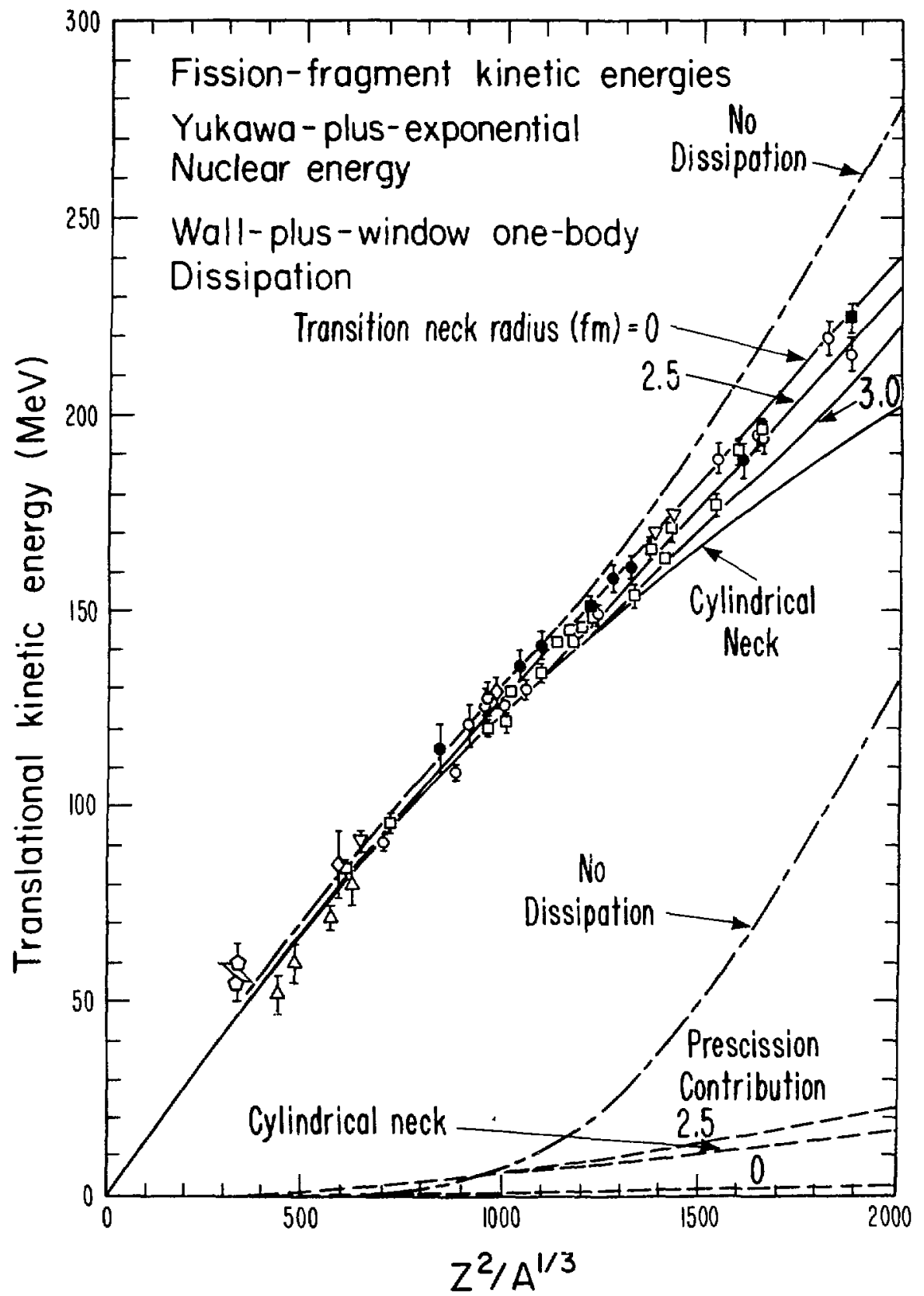

Fig. 21. This is like Fig. 15 but in the dynamical calculations a switch was made from the Wall Formula to the Wall-Plus-Window formula when the neck radius had reached the value indicated by the labels on the solid curves. "Cylindrical Neck" means the switch was made at the instant of first appearance of a constriction. The lower pari of the flgure shows the kinetic energy of the fragments at the instant of scission. Note the very large kinetic energles at scission when there is no dissipation, the very small values when the Wall Formula is used all the way to scission, and the moderate values when the switch is made before scission. After Ref. 15. 


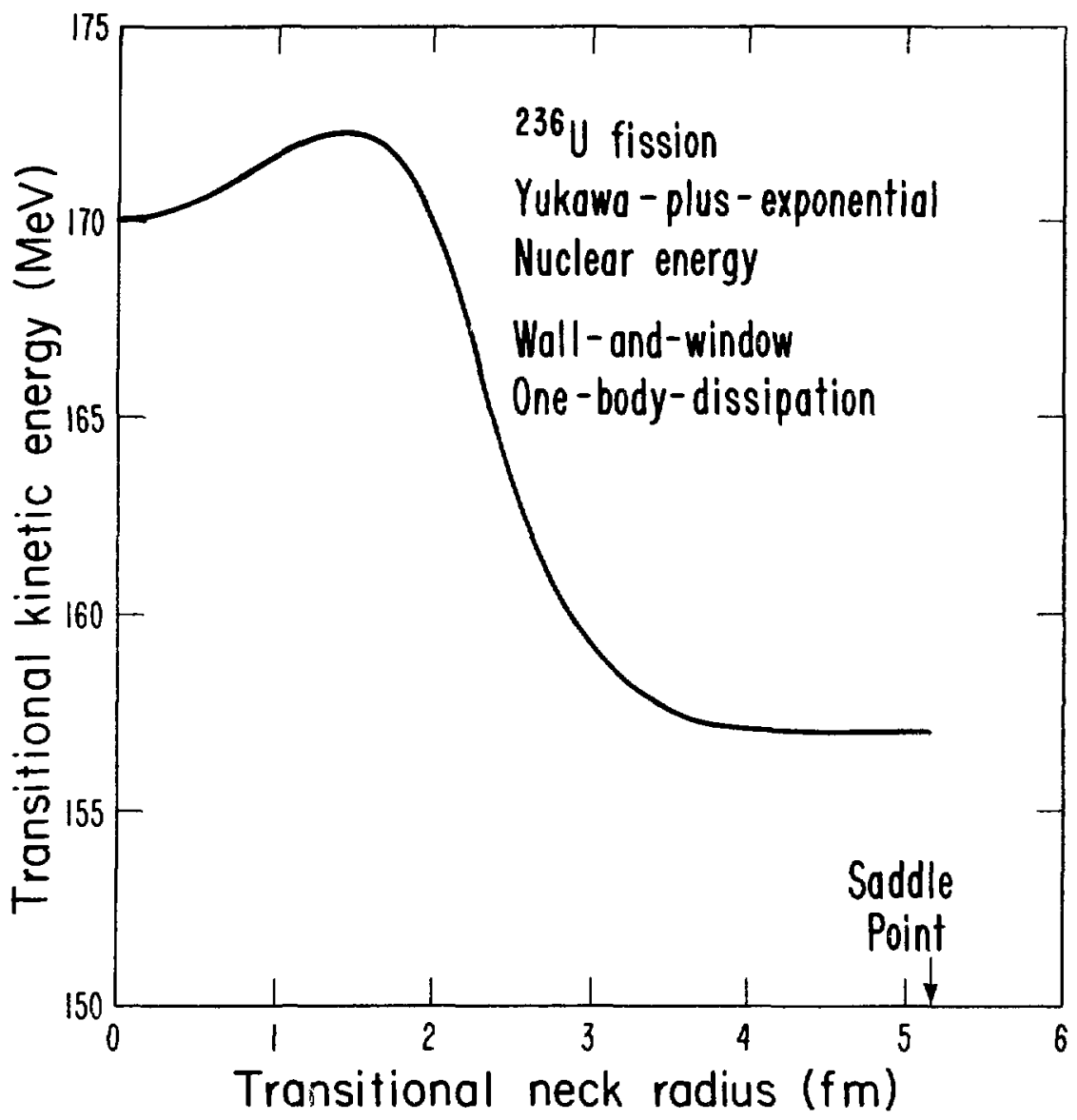

XBL $796-1829$

Fig. 22. The calculated kinetic energy of fragments from the fission of ${ }^{236} \mathrm{U}$ as a function of the neck radius at which a switch is made from the Wall Formula to the Wall-Plus-Window Formula. After Ref. 15. 


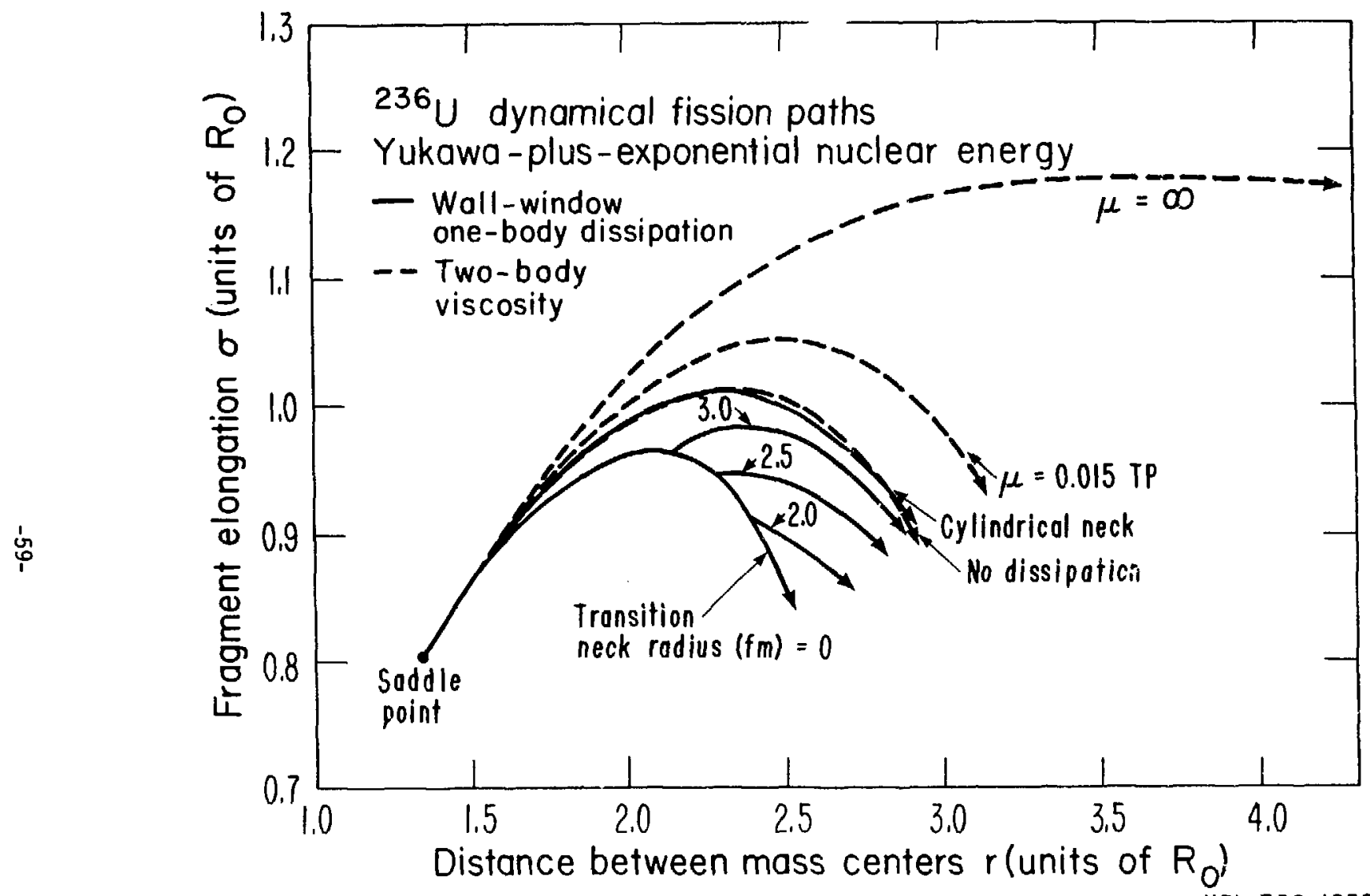

Fig. 23. This is like Fig. 17 and shows the effect on the fisston paths of switching from the Wall Formula to the Wa11-P1ns-Window Formula when the neck radius has reached the value Indicated by labels on the solid curves. For a reasonable transition neck radius the scission shapes (located by the arrowheads) are more compact than for the reference calculation without dissipation. After Ref. 15. 


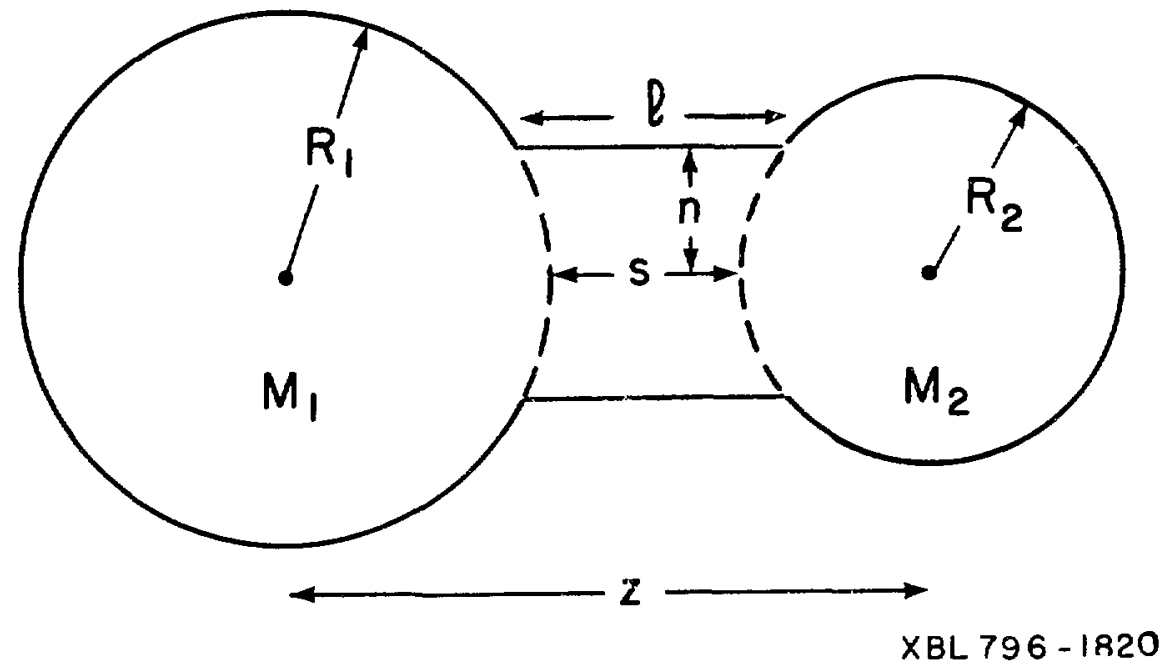

Fig. 24. The half-density contour of the nuclear configuration is parametrized as a dumb-bell consisting of two spheres with radii $R_{1}, R_{2}$ and a cylindrical neck of radius $n$. The distance between the surfaces of the spheres is $s$ and between their centers is $z$ (equal to $R_{1}+R_{2}+s$ ). The length of the rieck is $l \approx s+n^{2} / 2 \bar{R}$. 

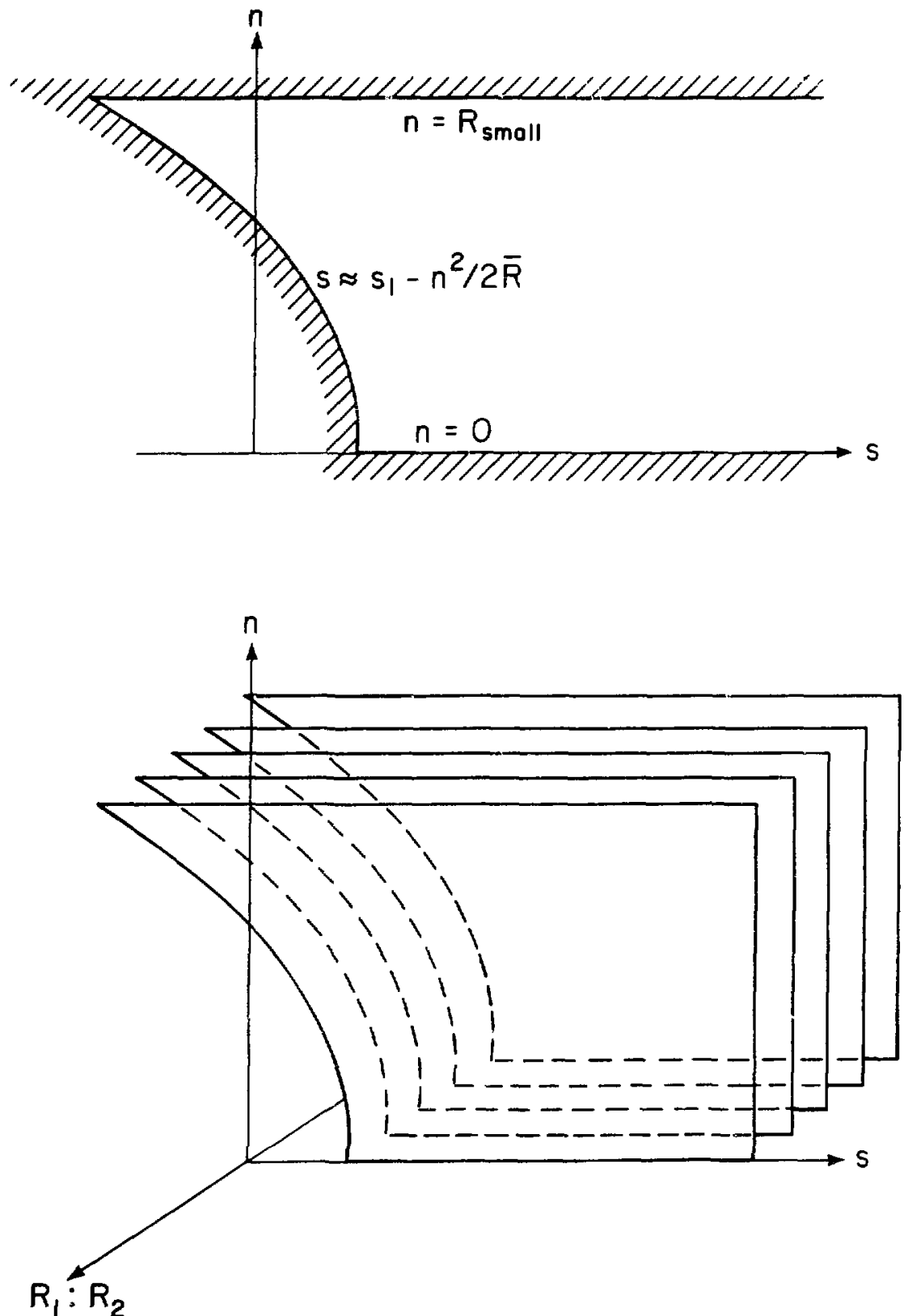

XBL $796-1822$

Fig. 25. A sketch of the configuration space of the separation (s) and neck radius $(n)$ degrees of freedom. The configuration space is bounded by the line $n=0$ (zero neck radius), the curve $s \approx s_{1}-n^{2} / 2 \bar{R}$ (the "geometrical" boundary) and a line $n=R_{\text {gmall }}$ (where the radius of the neck becomes equal to the smaller one of the two spheres). The lower part of the figure indicates how the asymmetry degree of freedom $\left(R_{1}: R_{2}\right)$ would be included. 


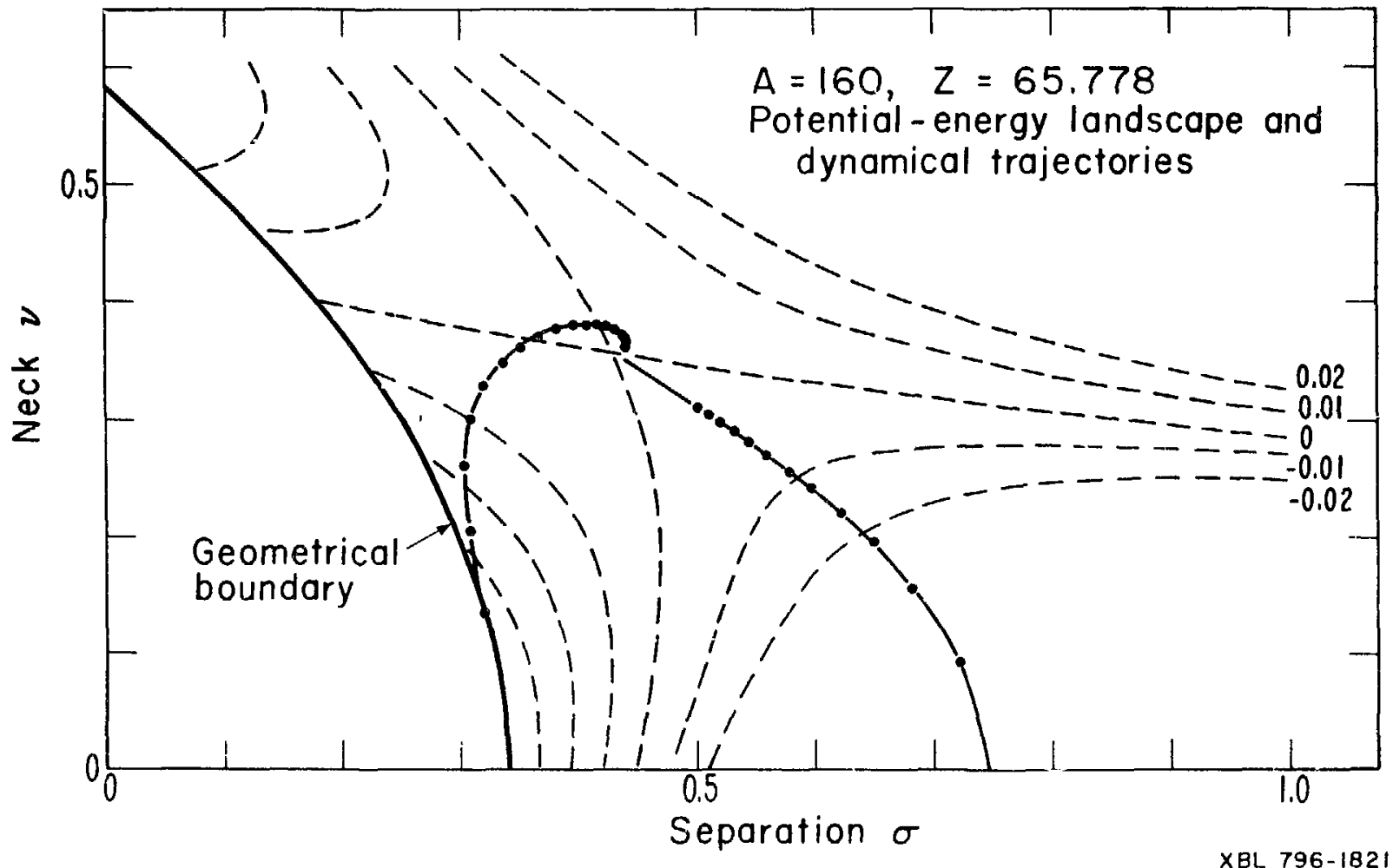

Fig. 26. The potential-energy landscape 1:1 the space of the neck and separation degrees of $f$ reedom for a system with mass number $A=160$ and $Z=65.778$. Equation (57a) was used to construct the equipotential 11nes, which are spaced at intervals of 0.01 in $\varepsilon$. Two trajectories are shown, corresponding to the dynamical time evolution of the system. One is a part of the critical collision trajectory that would end up at the saddle point, the other is a fission trajectory. The dots are spaced at time intervals of $0.1 u_{t}$. 


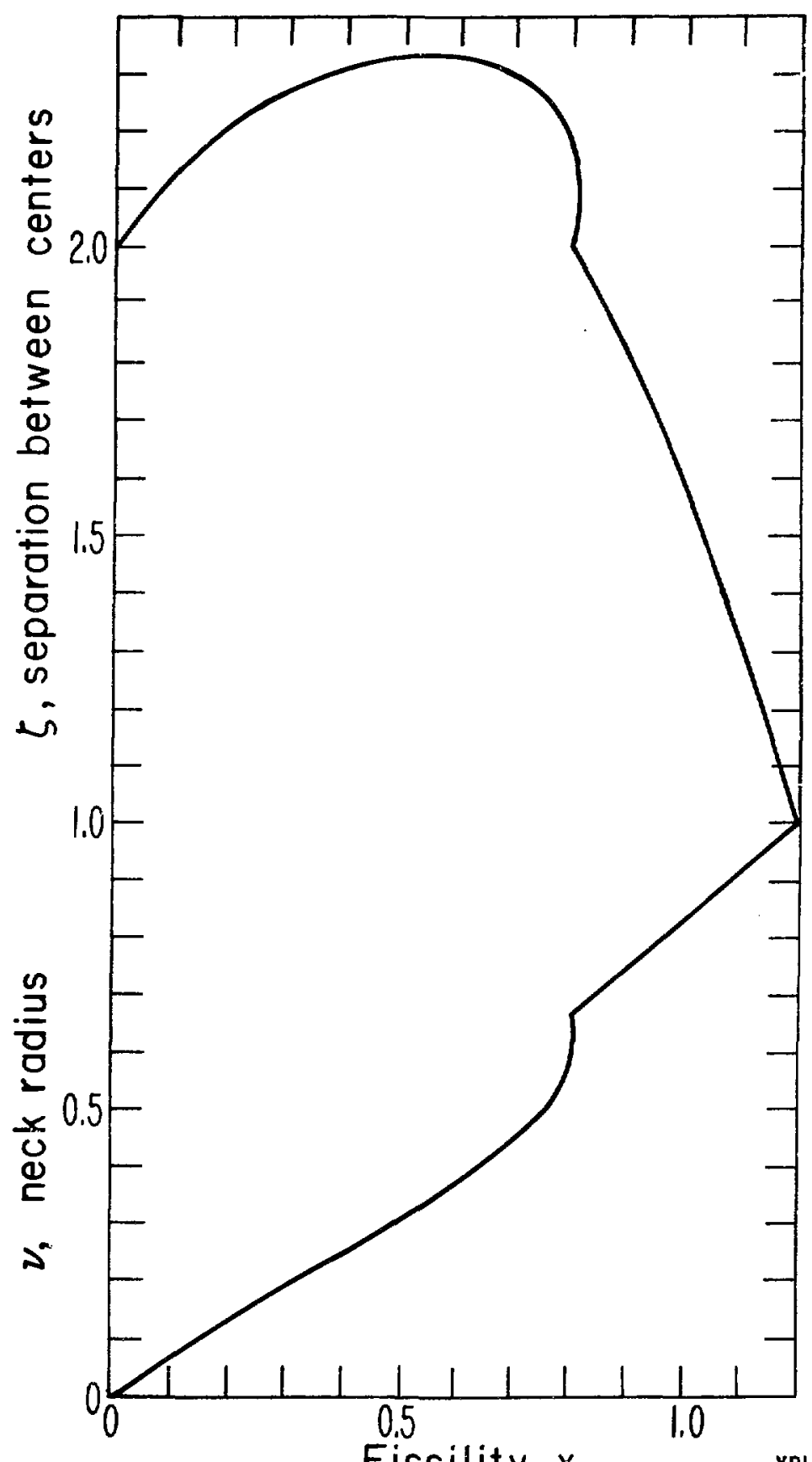

Fissility $x$

XBL $796-1824$

F1g. 27. The neck radius, $n$, and the separation between centers, $z$, both in units of $2 \bar{R}$, are plotted for fission saddle-point shapes as functions of the fissility parameter $x$. The approximations on which the calculations are based assume a small neck but the curves are displayed beyond the range of their expected validity, up to $x \approx 1$. 


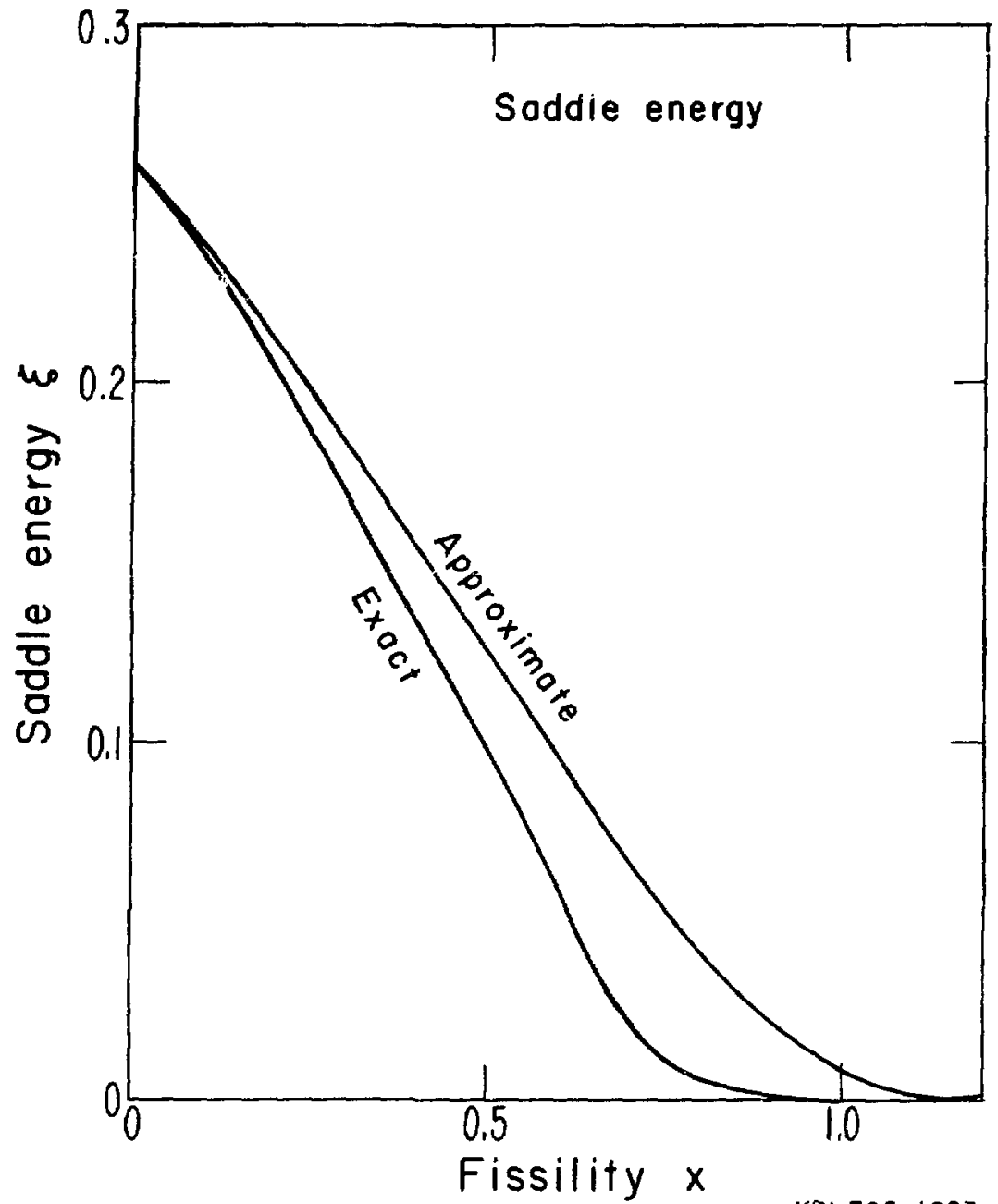

XBL $796-1823$

Fig. 28. A comparison of the exact saddle point energies with those obtained in our small-neck, dumb-bell approximation. The energies are plotted conventionally with respect to the energy of a single sphere and in units of that sphere's surface energy. The fission barrier energies, calculated on the basis of the approximate curve, could be brought into fair agreement with the exact result by a sifght re-scaling of the fissility parameter $\mathbf{x}$. 


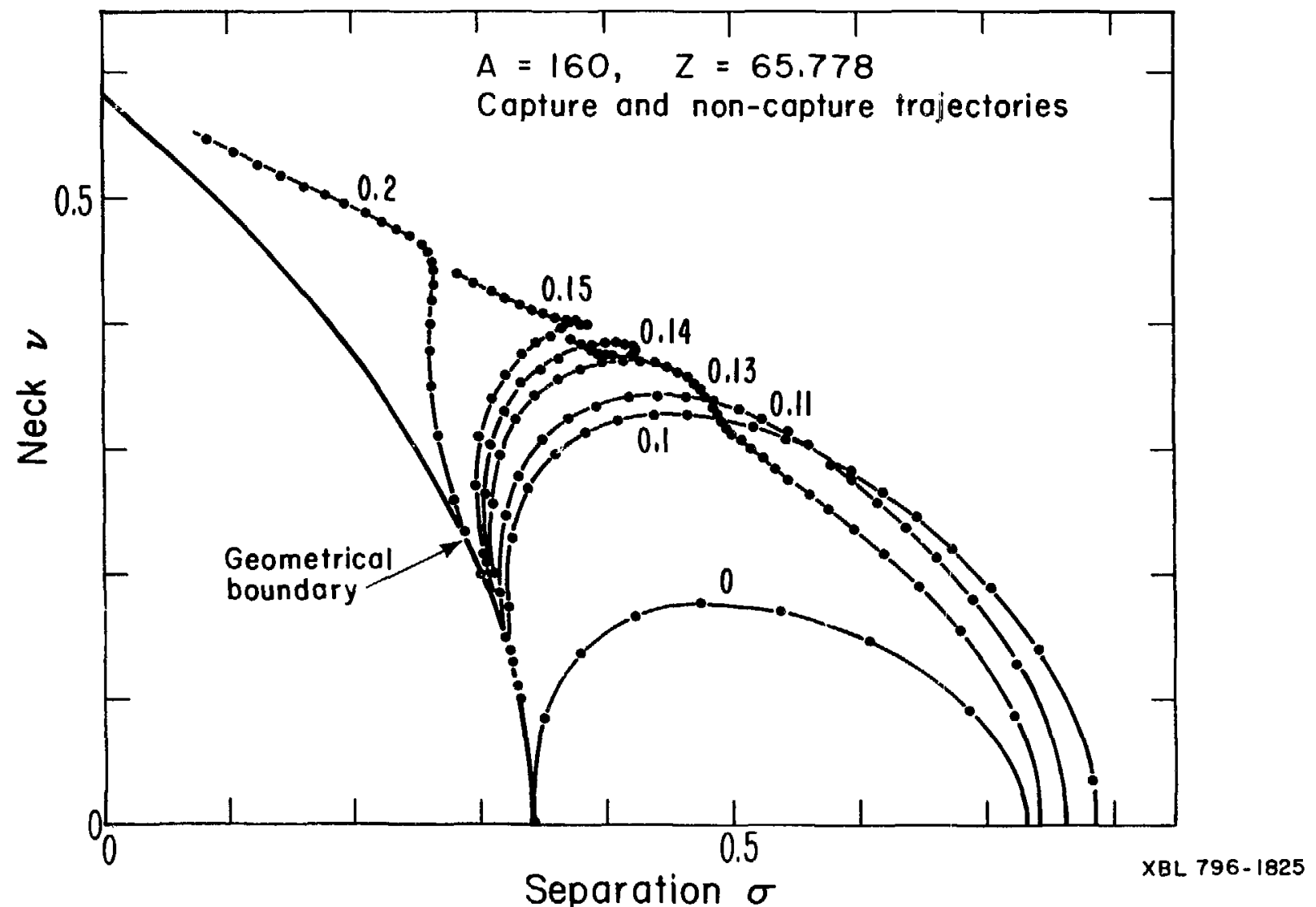

Fig. 29. Dynamical trajectories of colliding nuclei in the configuration space of separation, $\sigma$, and neck radius, $\nu$. The approach of the nuclei proceeds from right to left along the $\sigma$-axis. This is followed by a geometrical neck growth along the geometrical boundary until a peeling-off point. After that the trajectories divide into two classes, depending on the value of $\nu$ at the moment of peeling of $f$ (shown as labels on the curves). For $v<0.1363$ the trajectories lead to re-disintegration. For $\nu>0.1363$ capture occurs inside the saddle point (marked by a cross). The dots along the trajectories correspond to time intervals of one tenth of the natural time unit $u_{t}$ appropriate for the system in question. 


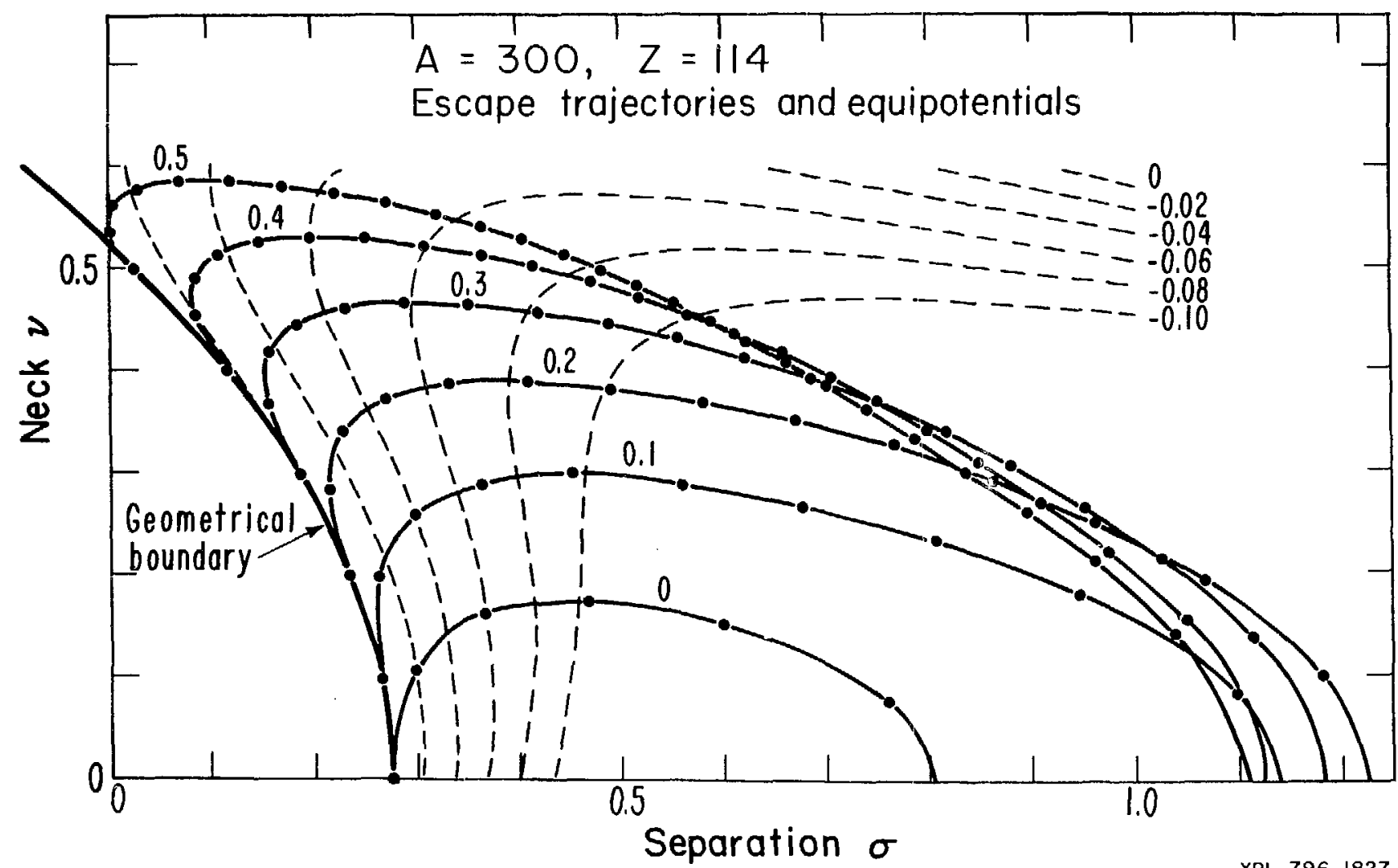

Fig. 30. This is similar to Fig. 29 but for a superheavy system with $A=300$. The dashed 1 ines are equipotentlals spaced at intervals of 0.02 times the energy unit $8 \pi \gamma \bar{R}^{2}$. The solid lines are trajectorles that peeled of the geometrical_boundary at the indicates values of $\nu$. The dots indicate intervals of 0.1 of the time unit $\rho \bar{v}^{2} / \gamma$. A11 the trajectories shown lead to re-disintegration. 


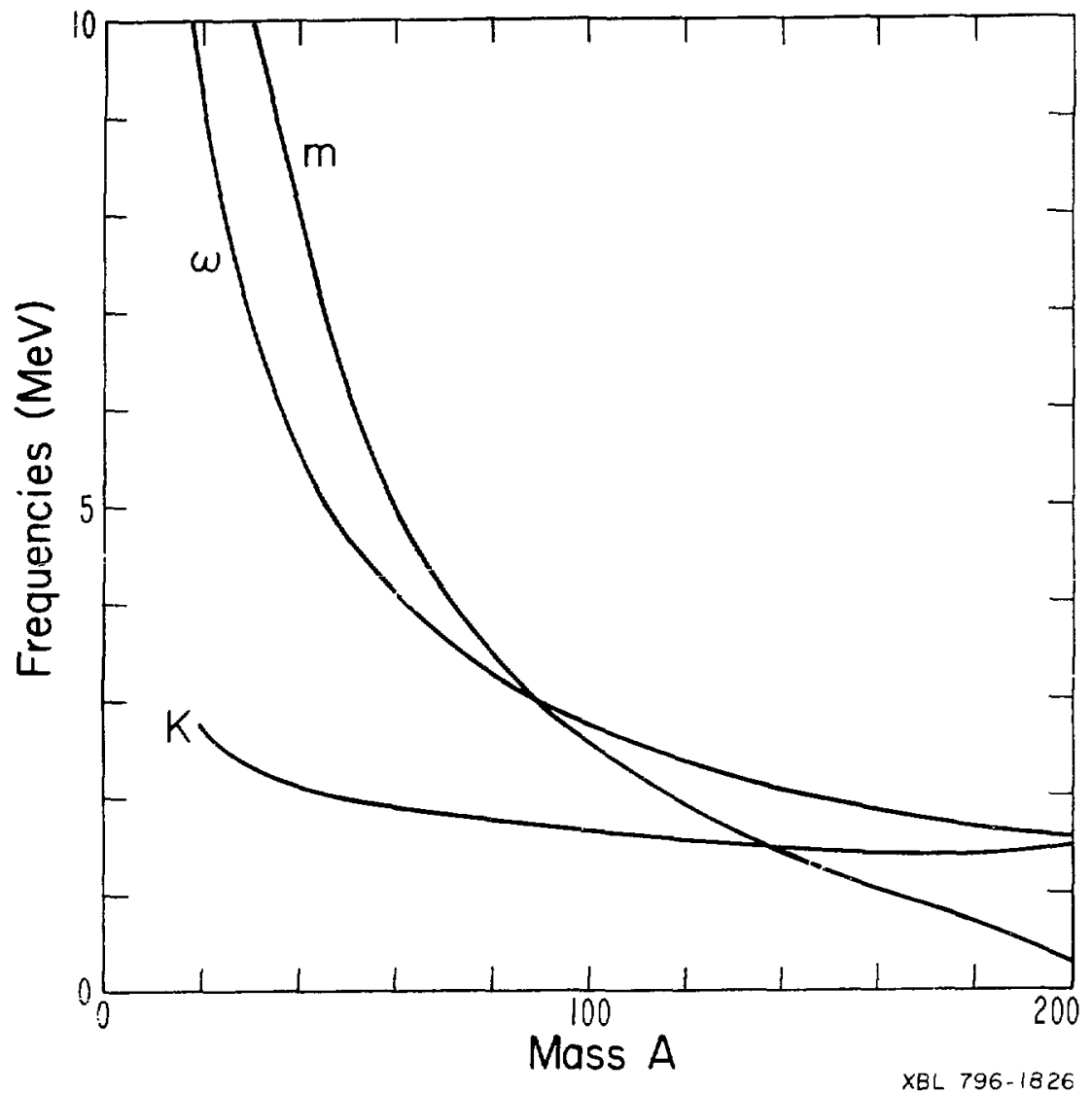

Fig. 31. The characteristic frequencies (expressed as energies by multiplication with $h$ ) for small motion near the saddle point. The plots are against the mass number $A$. The curve labeled $\omega$ refers to the frequency of the stable mode, $K$ to its damping width, and $m$ to the unstable fission mode. 